Supporting Information for

\title{
Generation of an Extremely Bent Pyrene System Using Kinetic Stabilization
}

Baozhong Zhang, Robert A. Pascal, Jr., Yuming Zhao, Graham J. Bodwell*

1. ${ }^{1} \mathrm{H}$ and ${ }^{13} \mathrm{C}$ NMR Spectra

$\mathrm{S} 2-\mathrm{S} 50$

2. Calculated structures of 2a-e, 3a-e and 13a-e

S51-S57

3. Computational Details and Cartesian Co-ordinates for Calculated Structures of $2 \mathbf{a}-\mathbf{e}, 3 \mathbf{a}-\mathbf{e}, 13 \mathbf{a}-\mathbf{e}$ and $7 \mathbf{a}-\mathbf{e}$

S58-S100 


\section{1. ${ }^{1} \mathrm{H}$ and ${ }^{13} \mathrm{C}$ NMR Spectra}

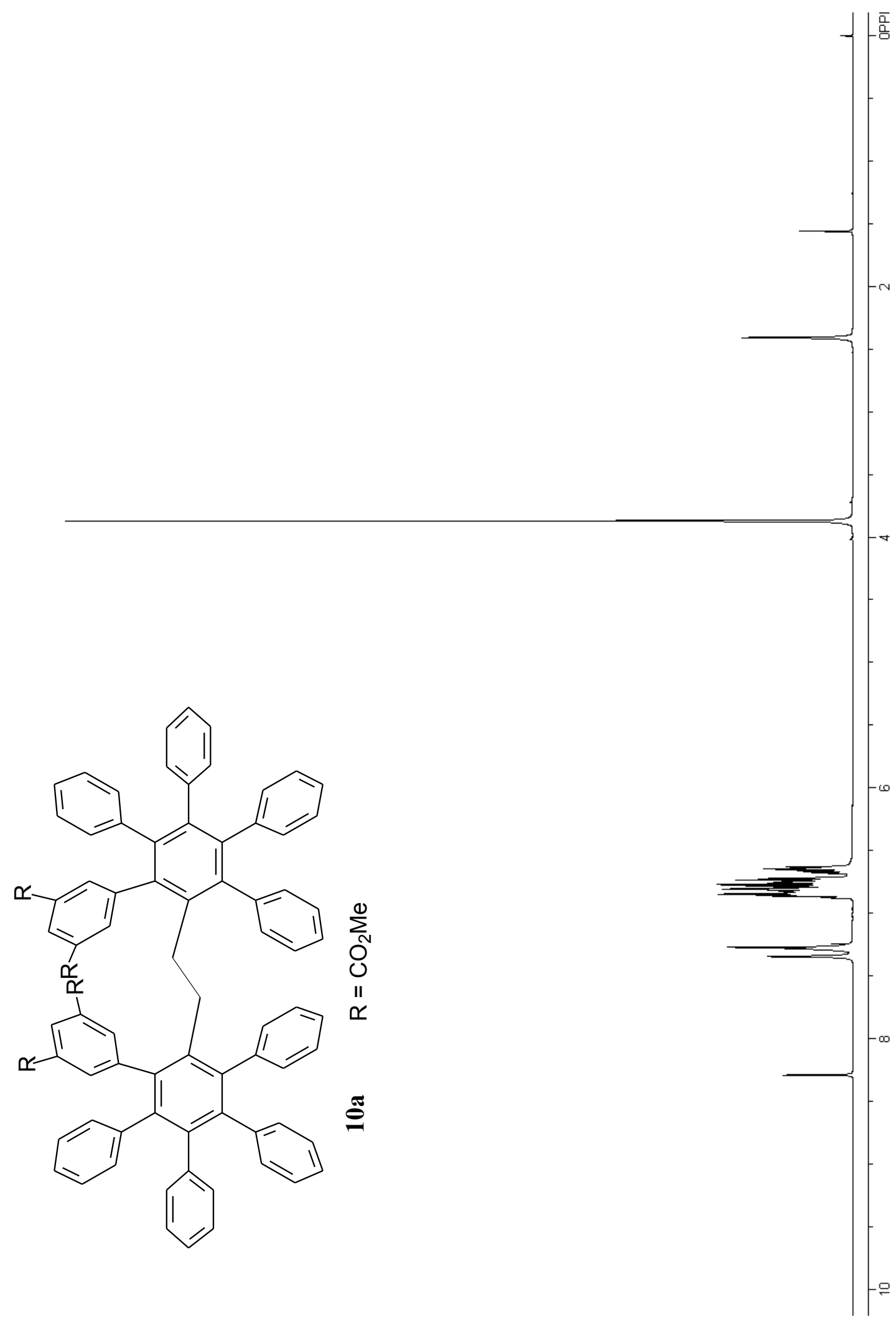




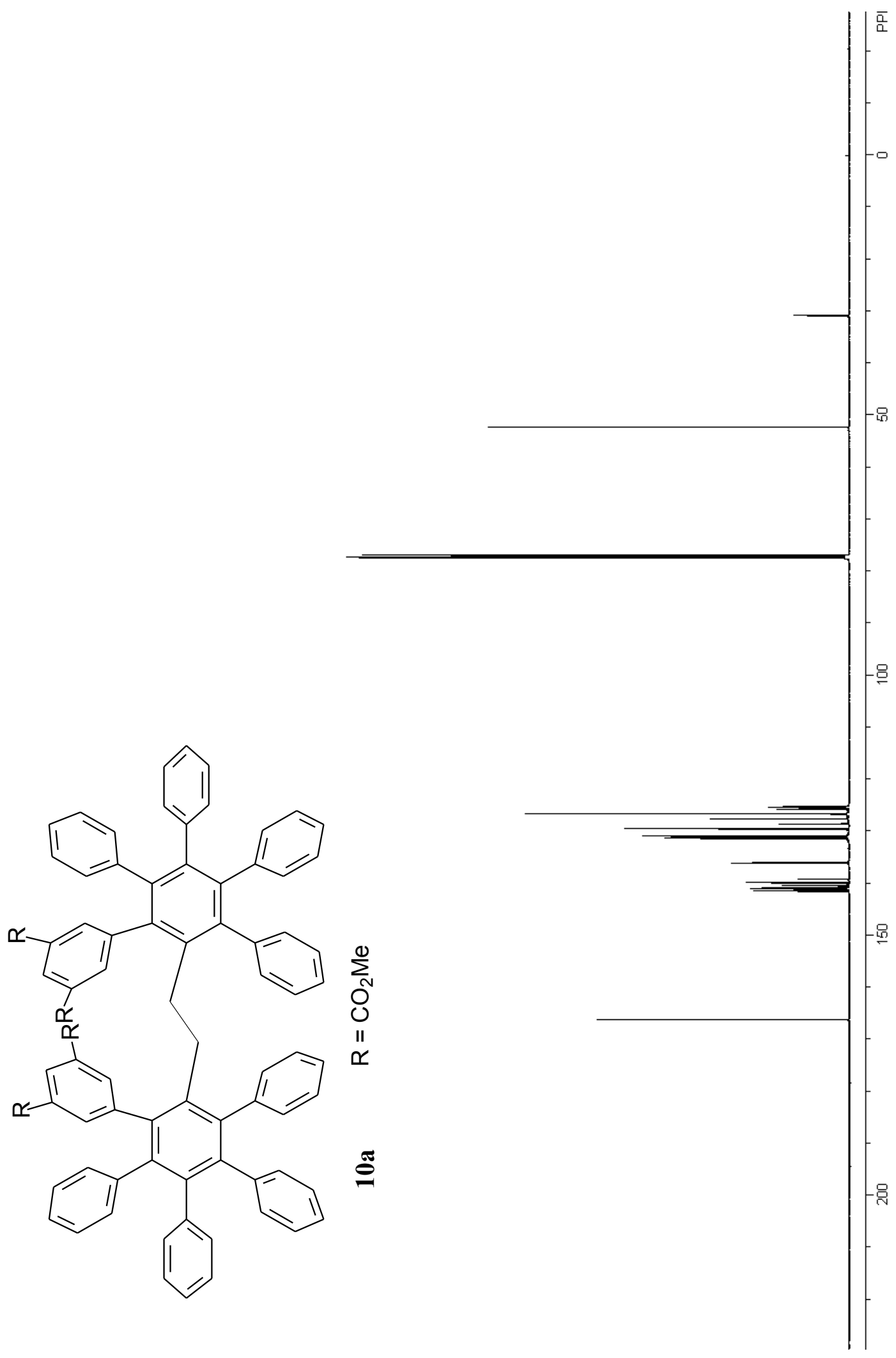




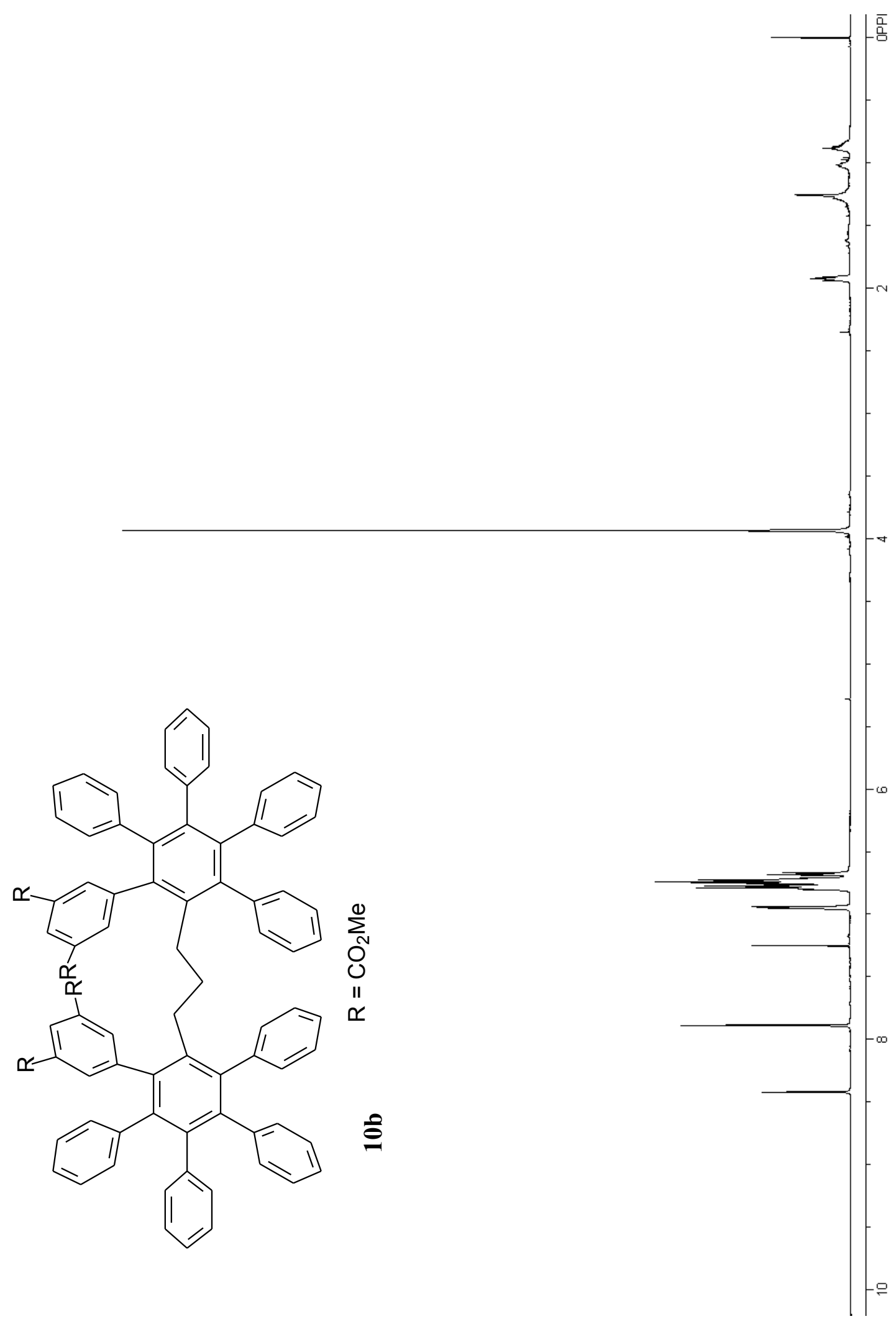




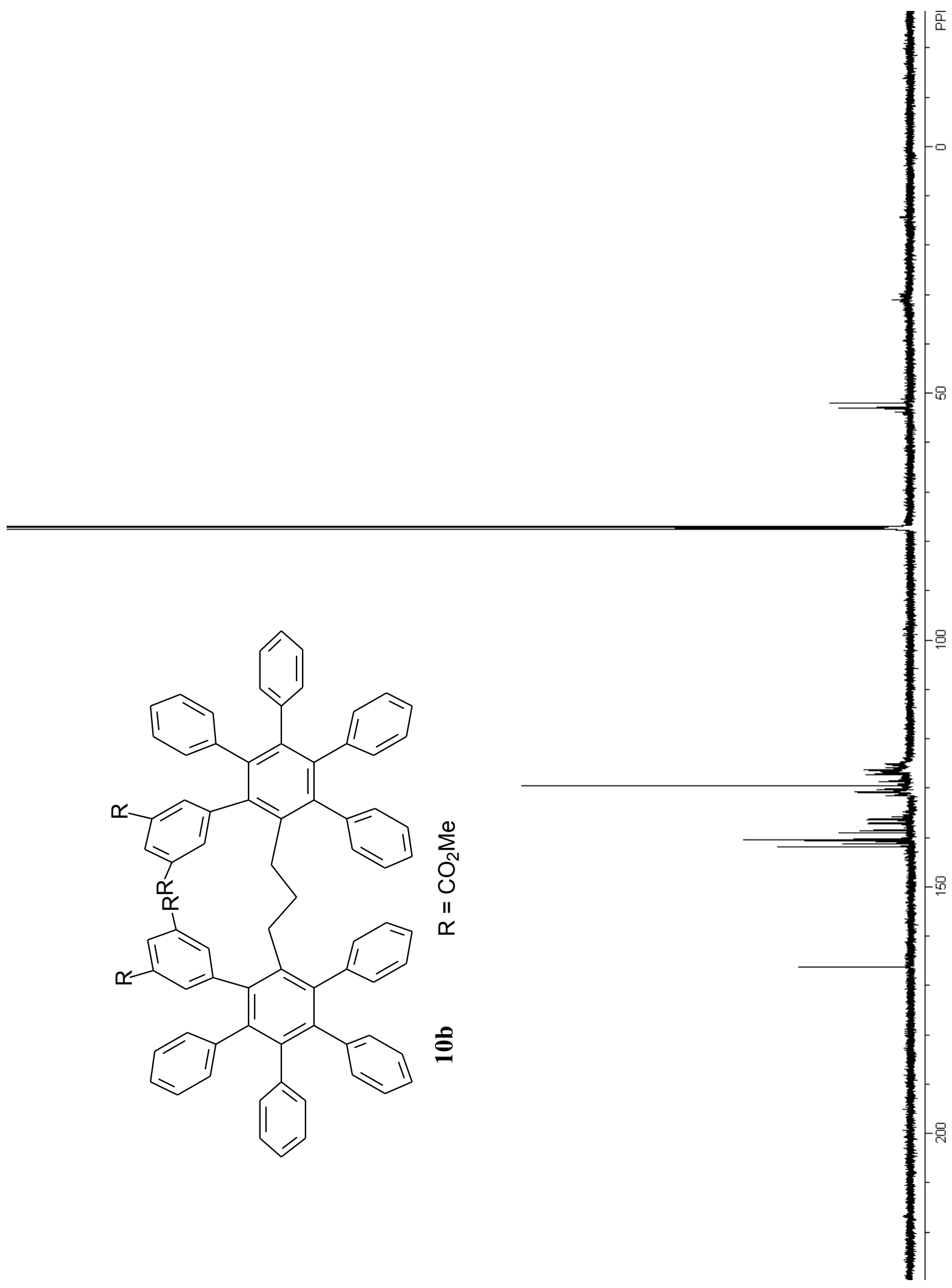




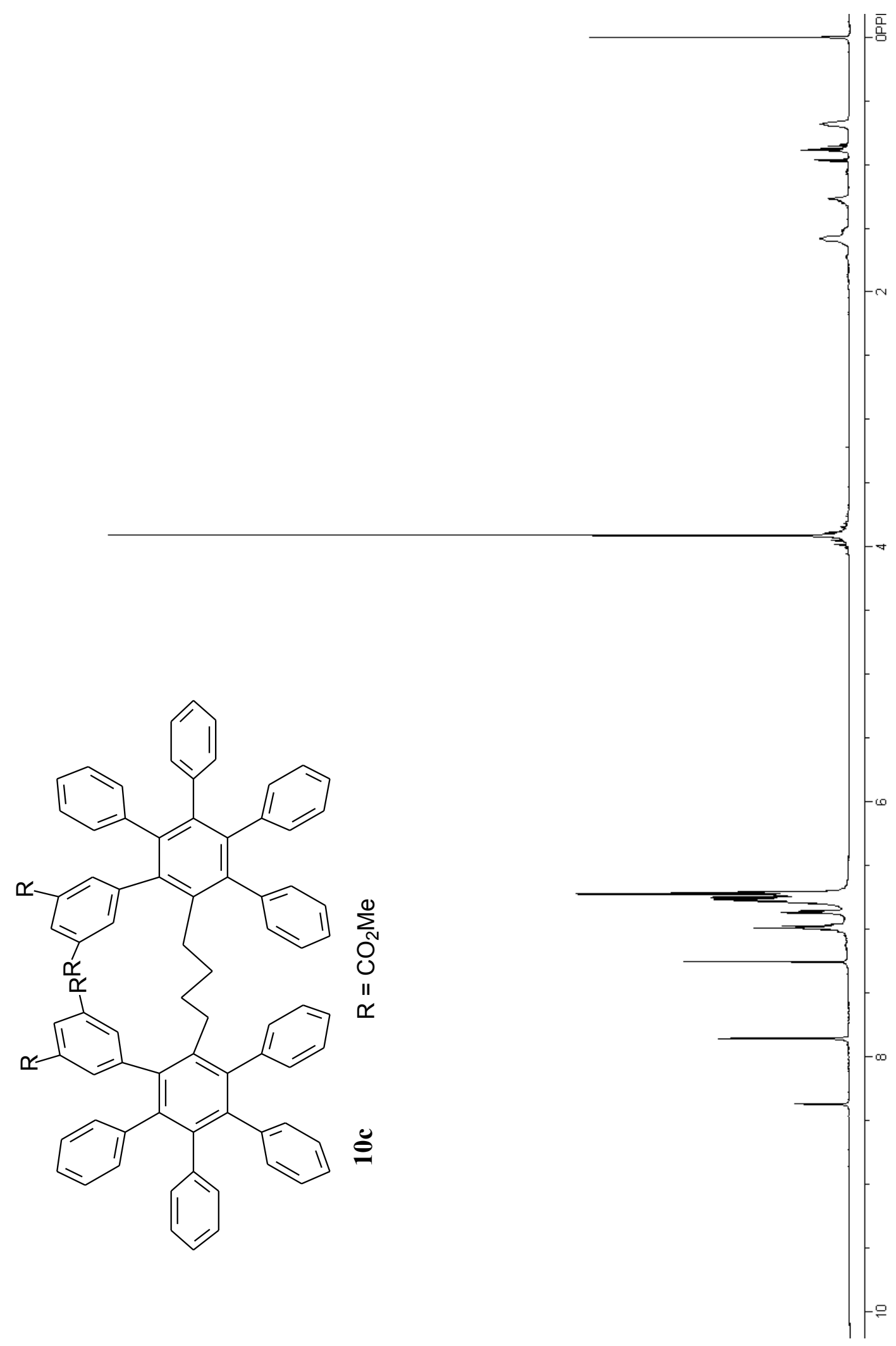




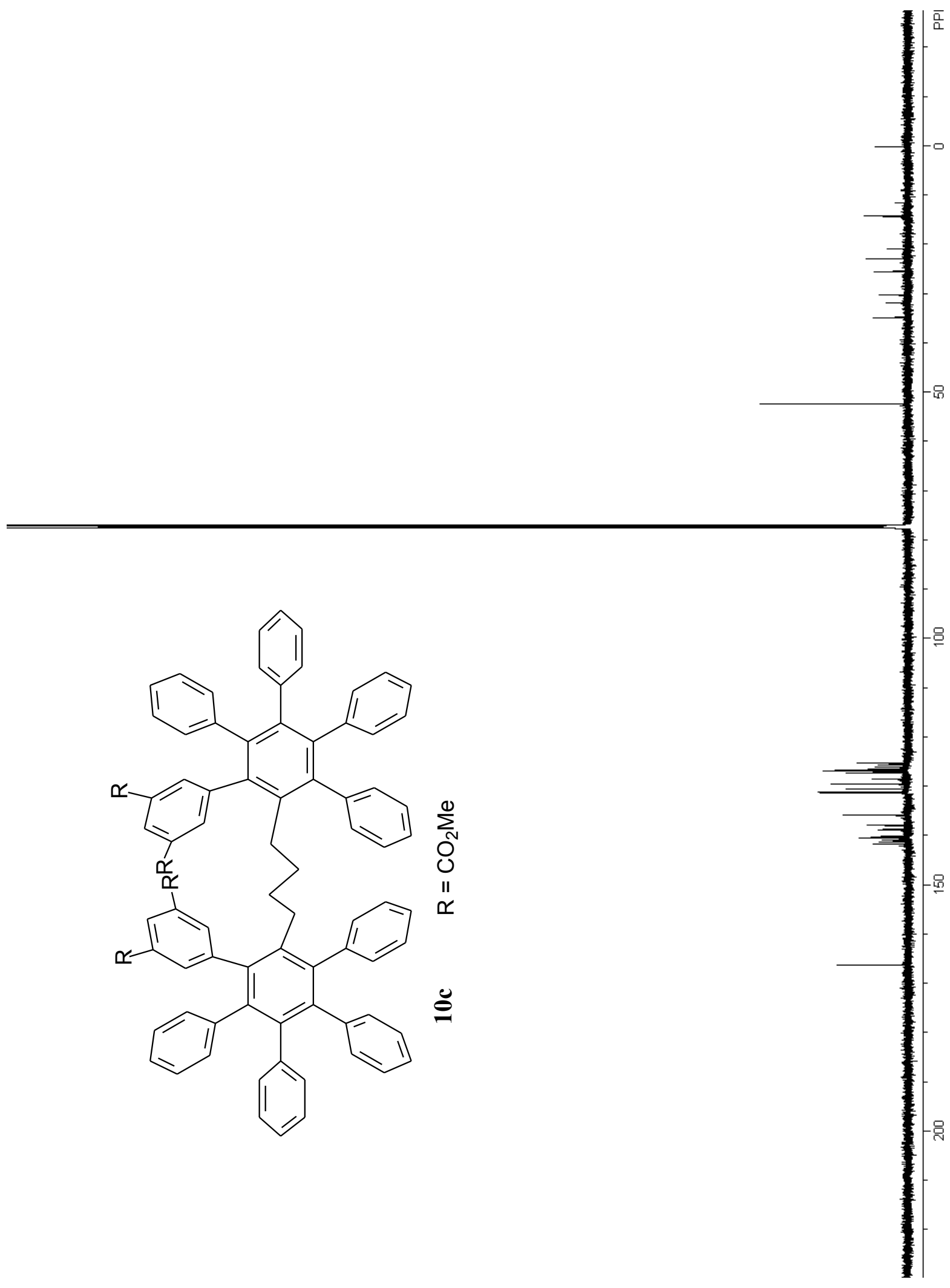




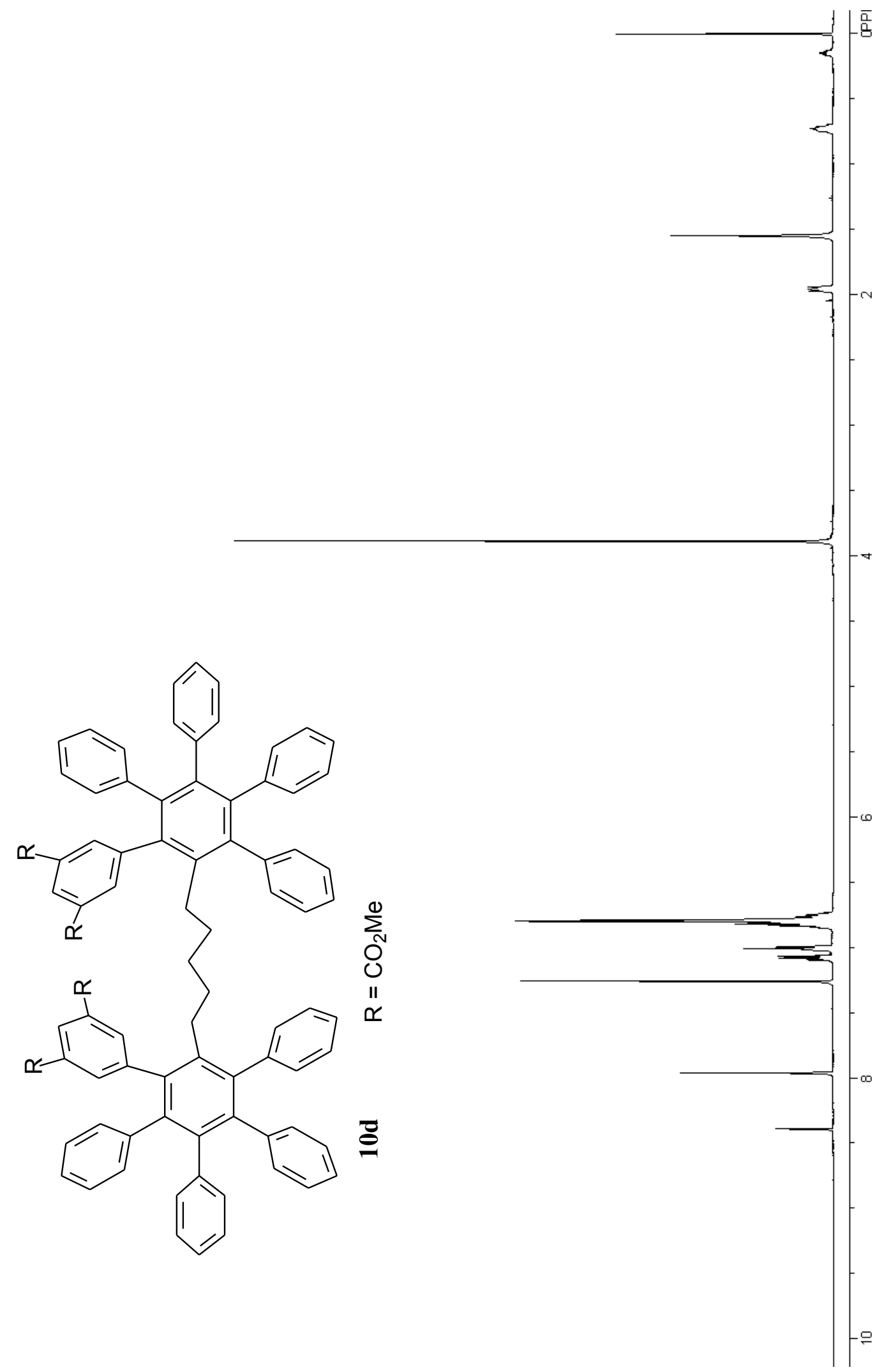




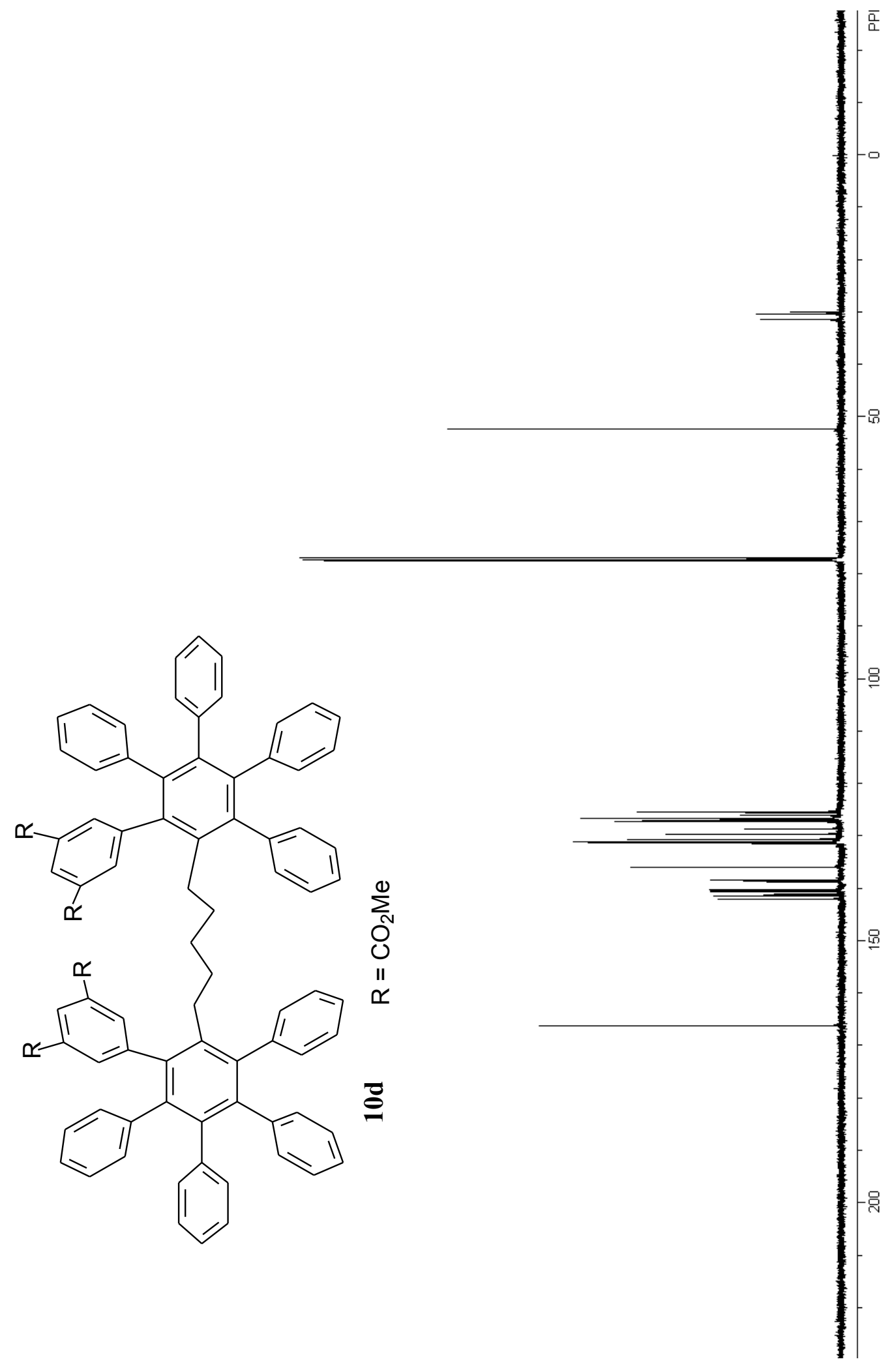




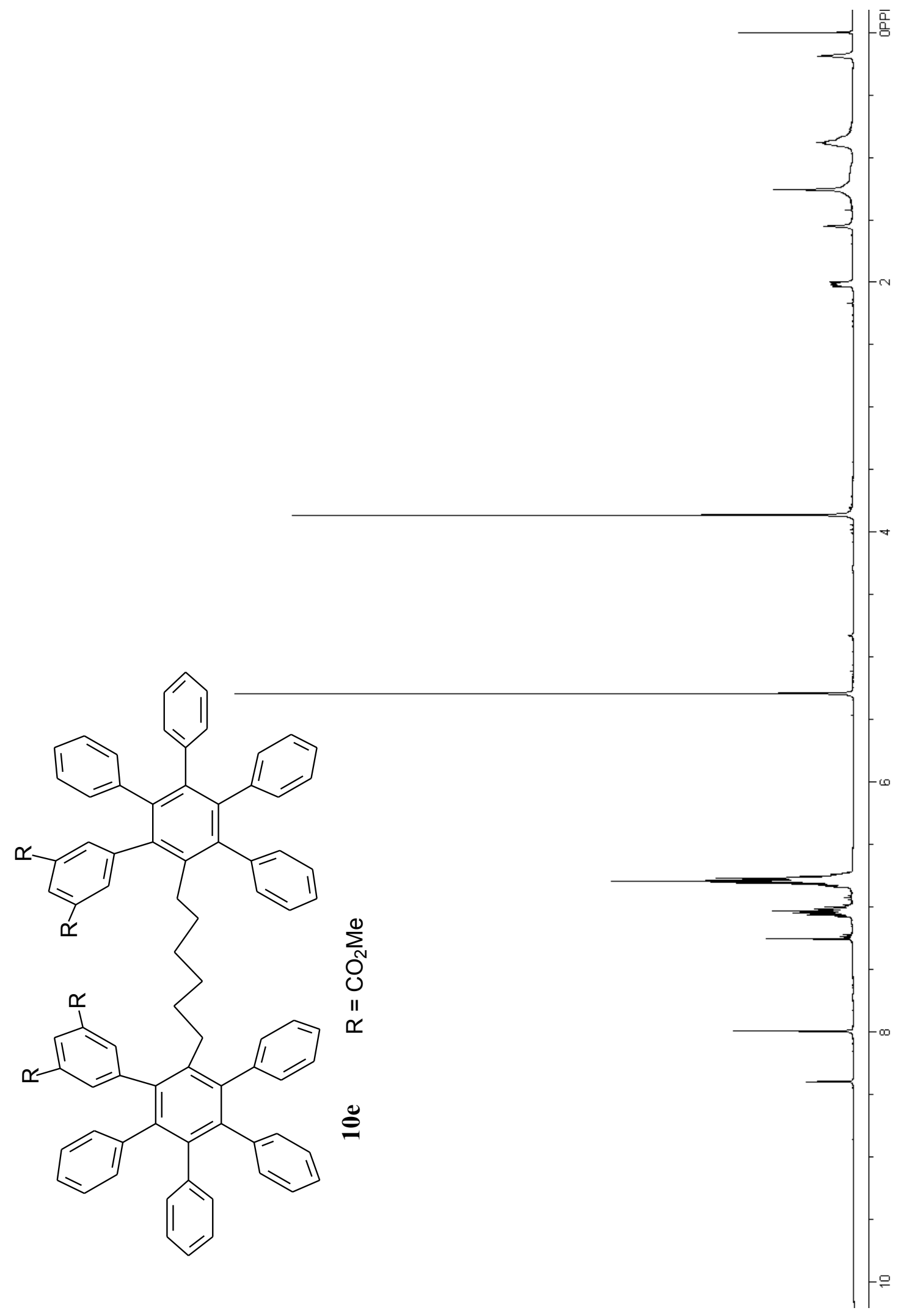




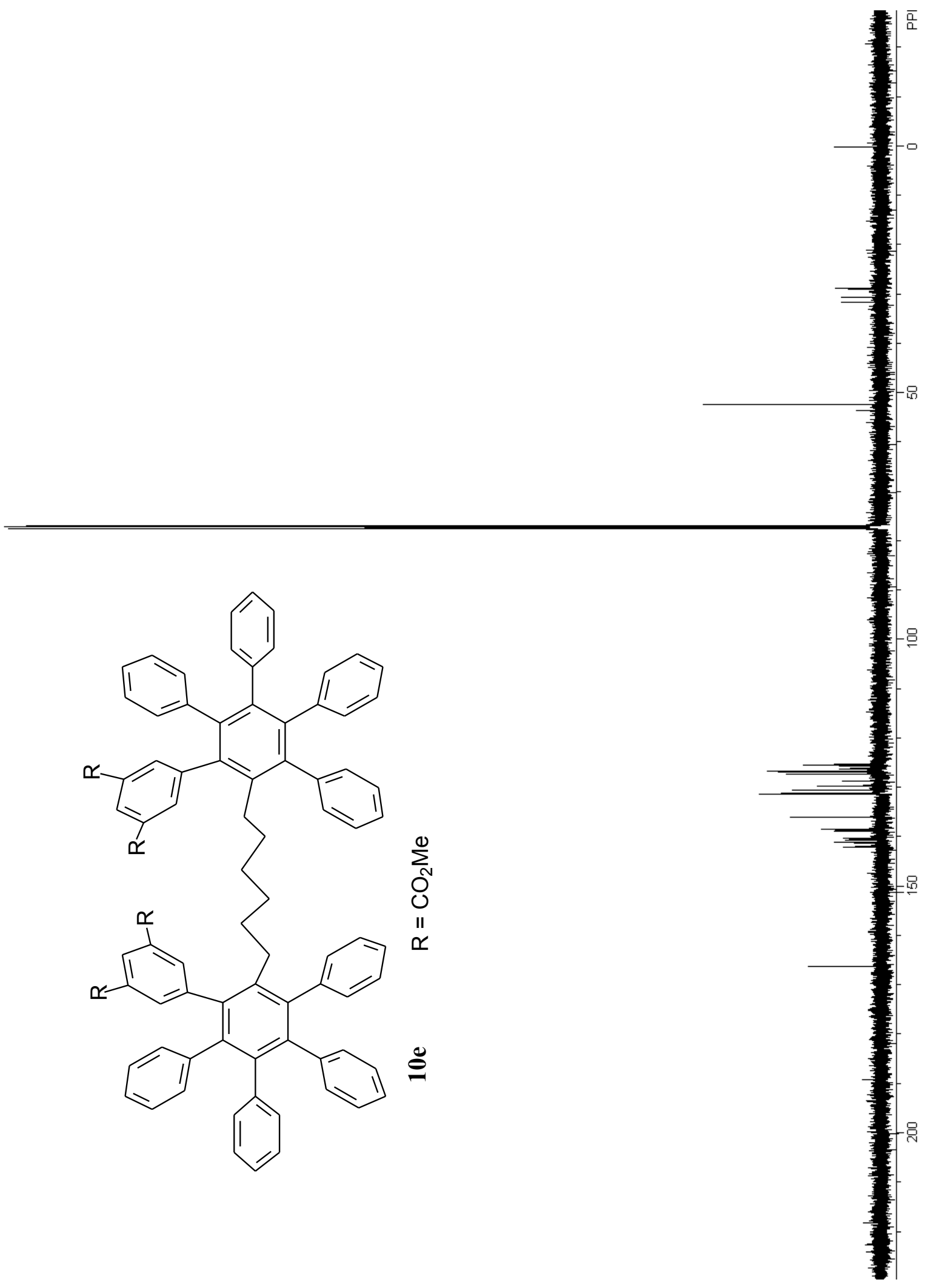

S-11 


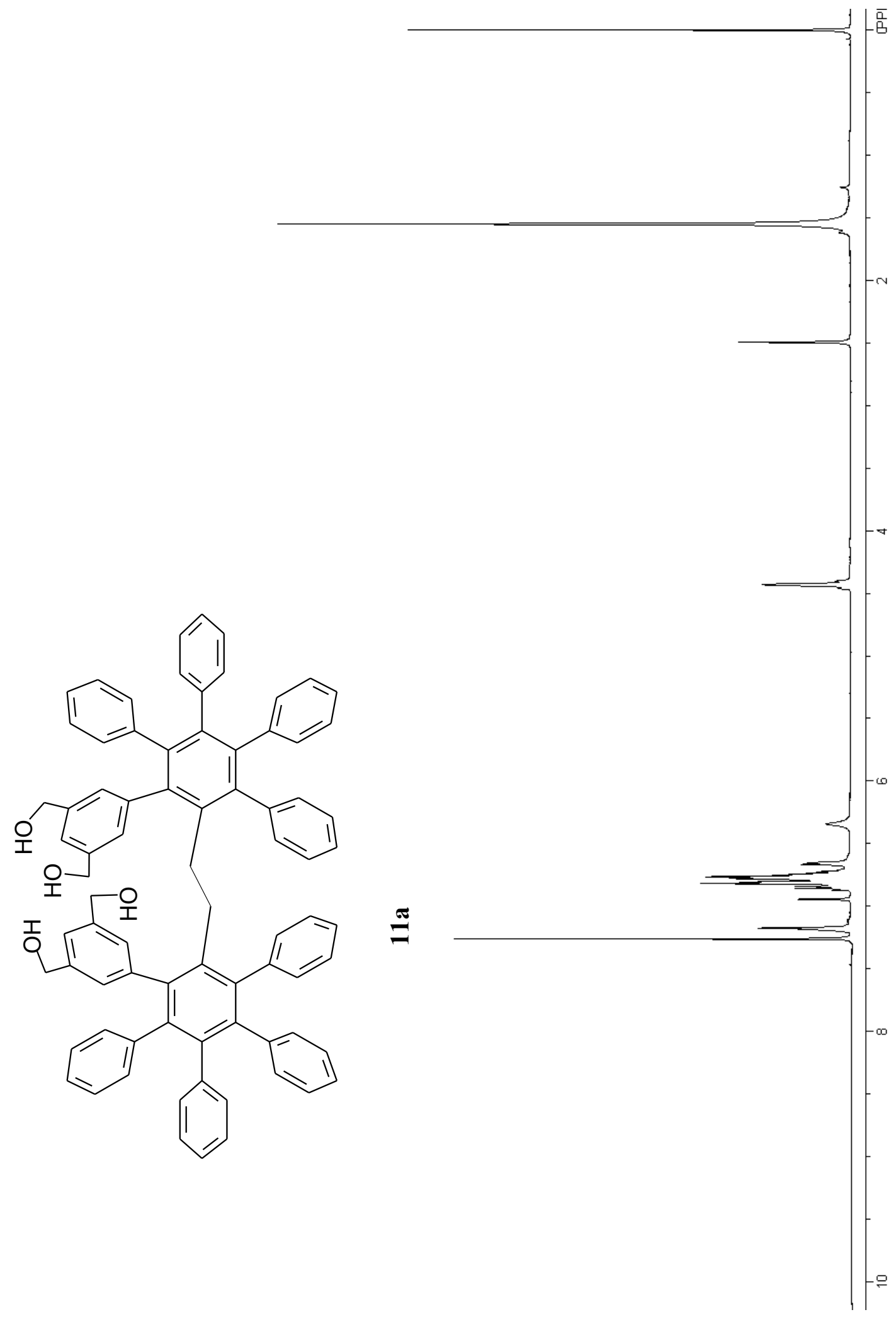

S-12 


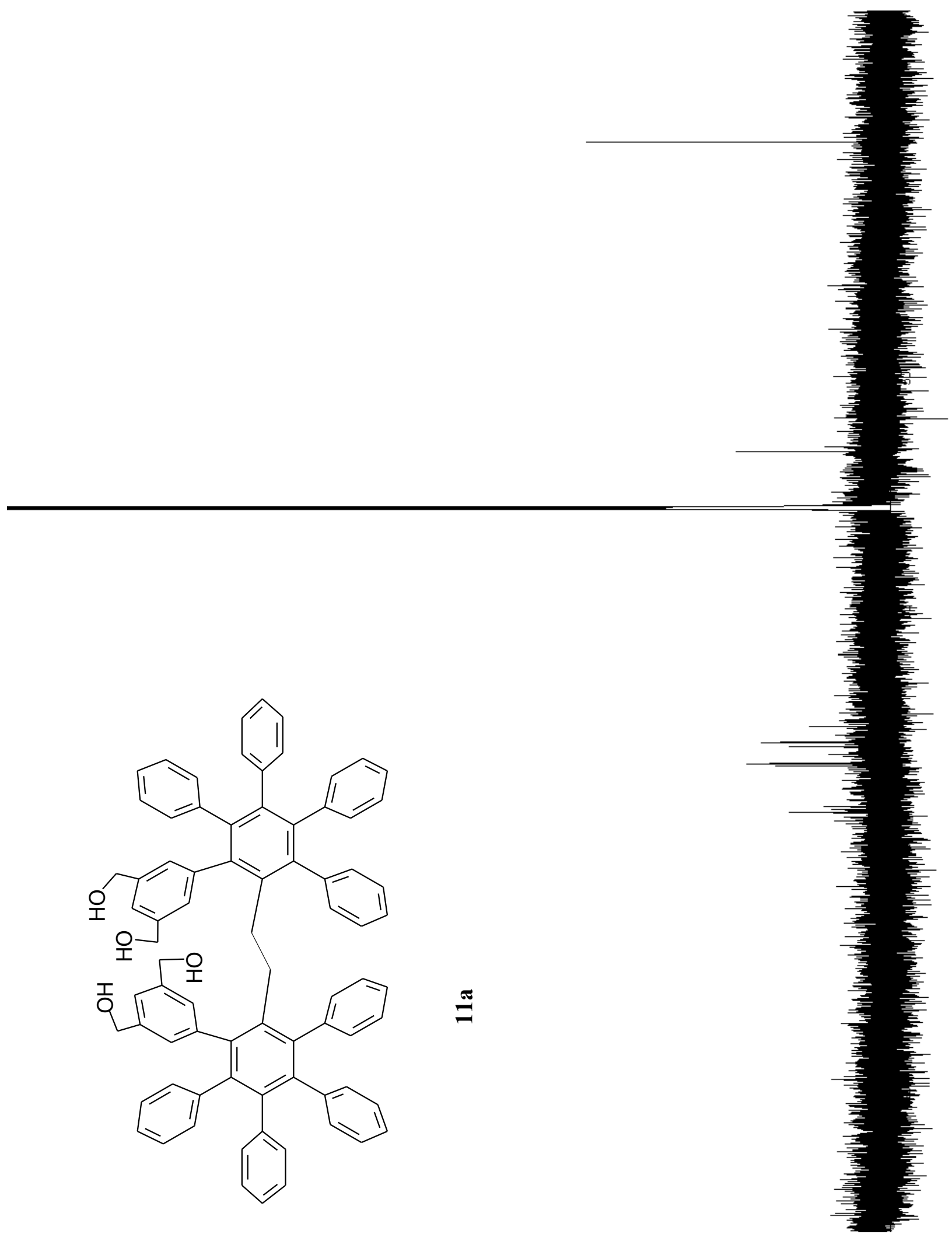




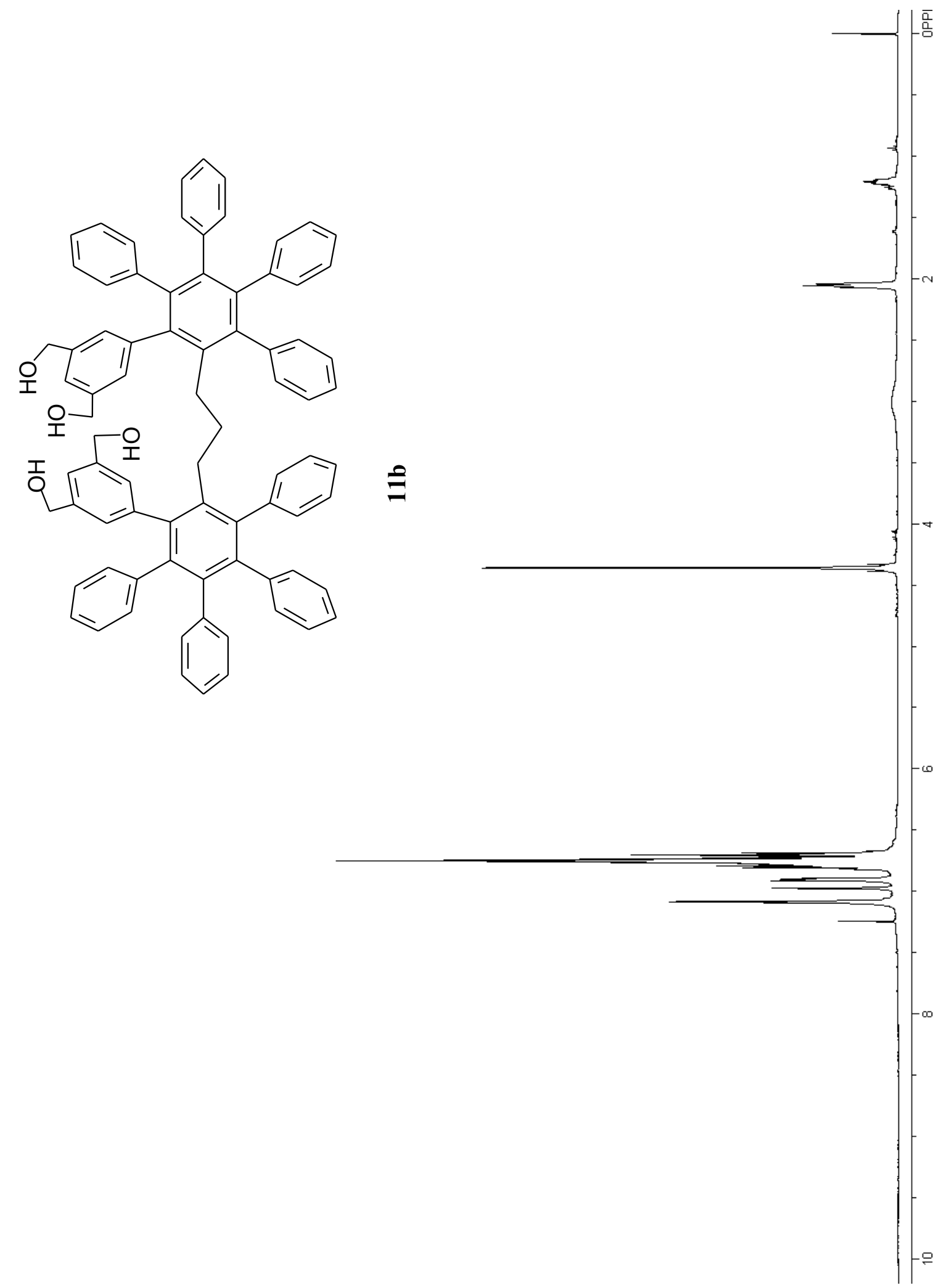




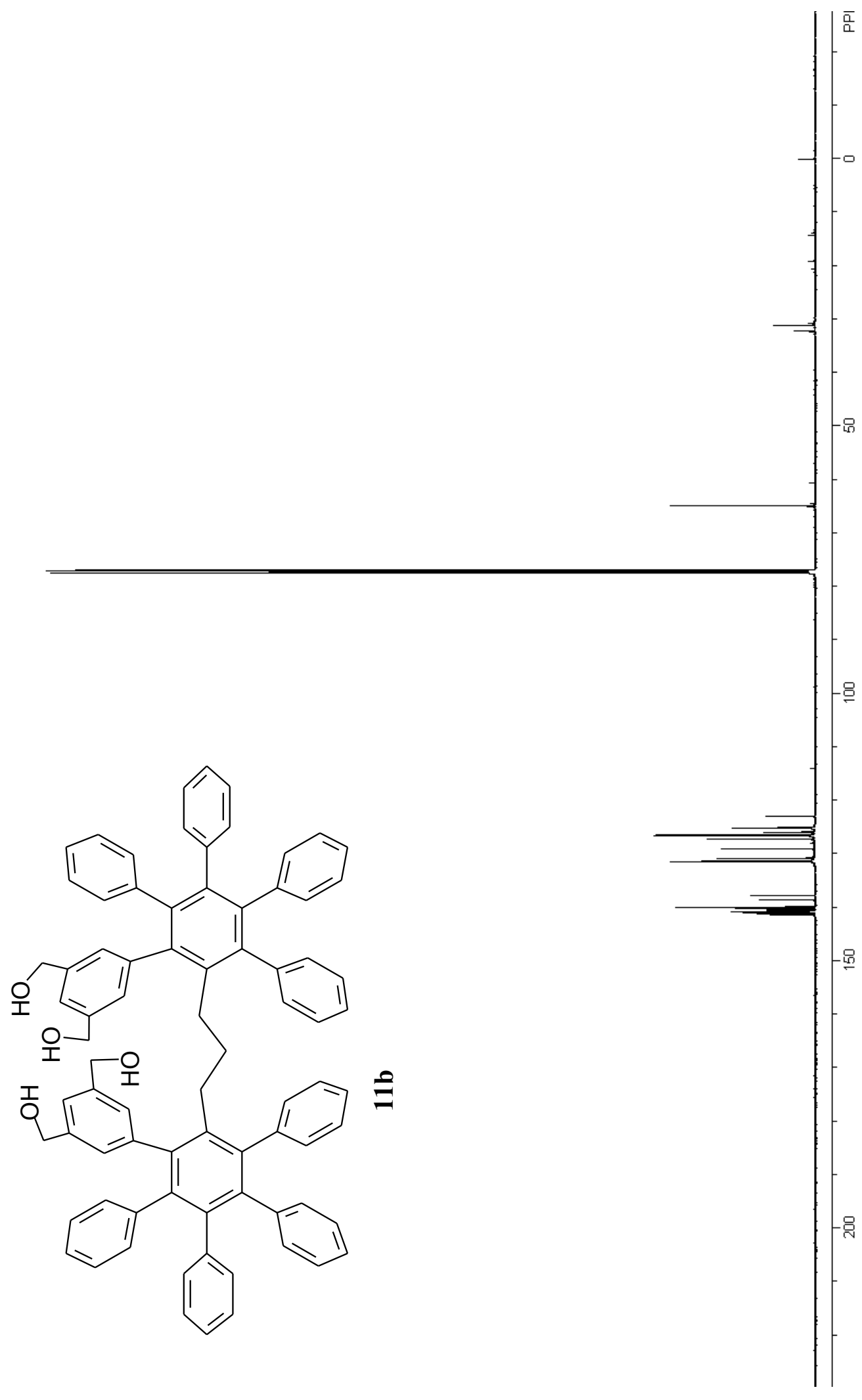




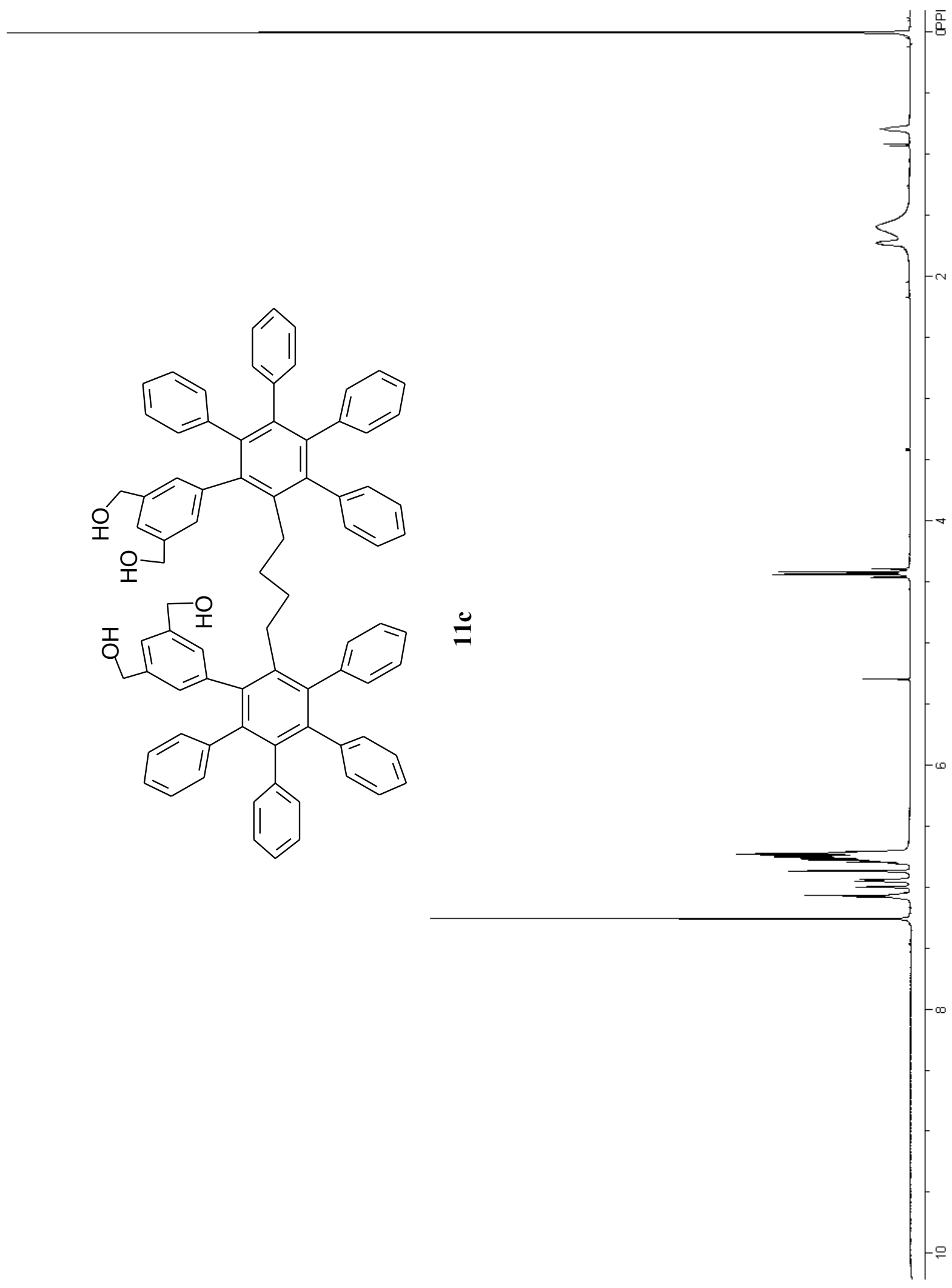

S-16 


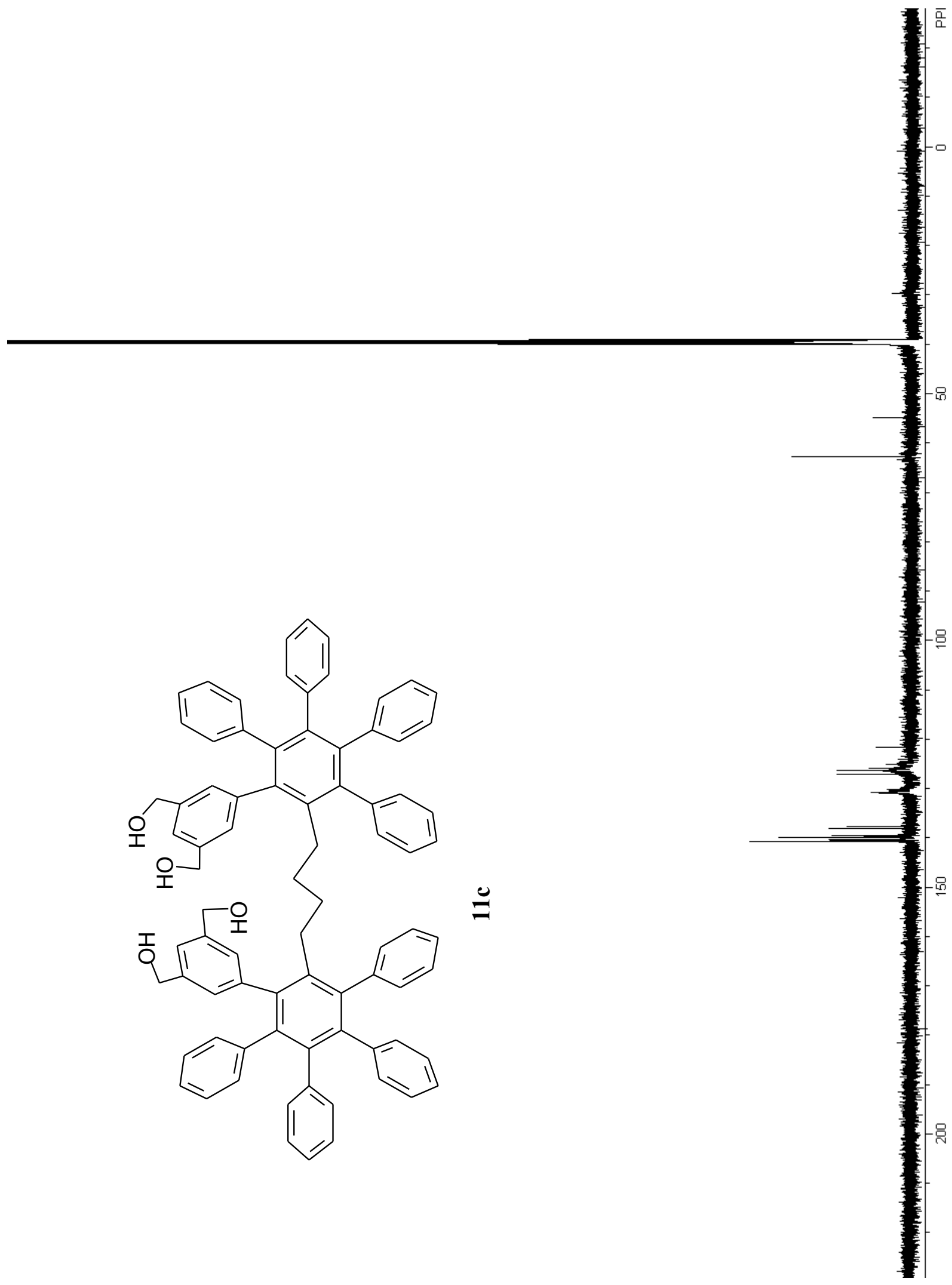




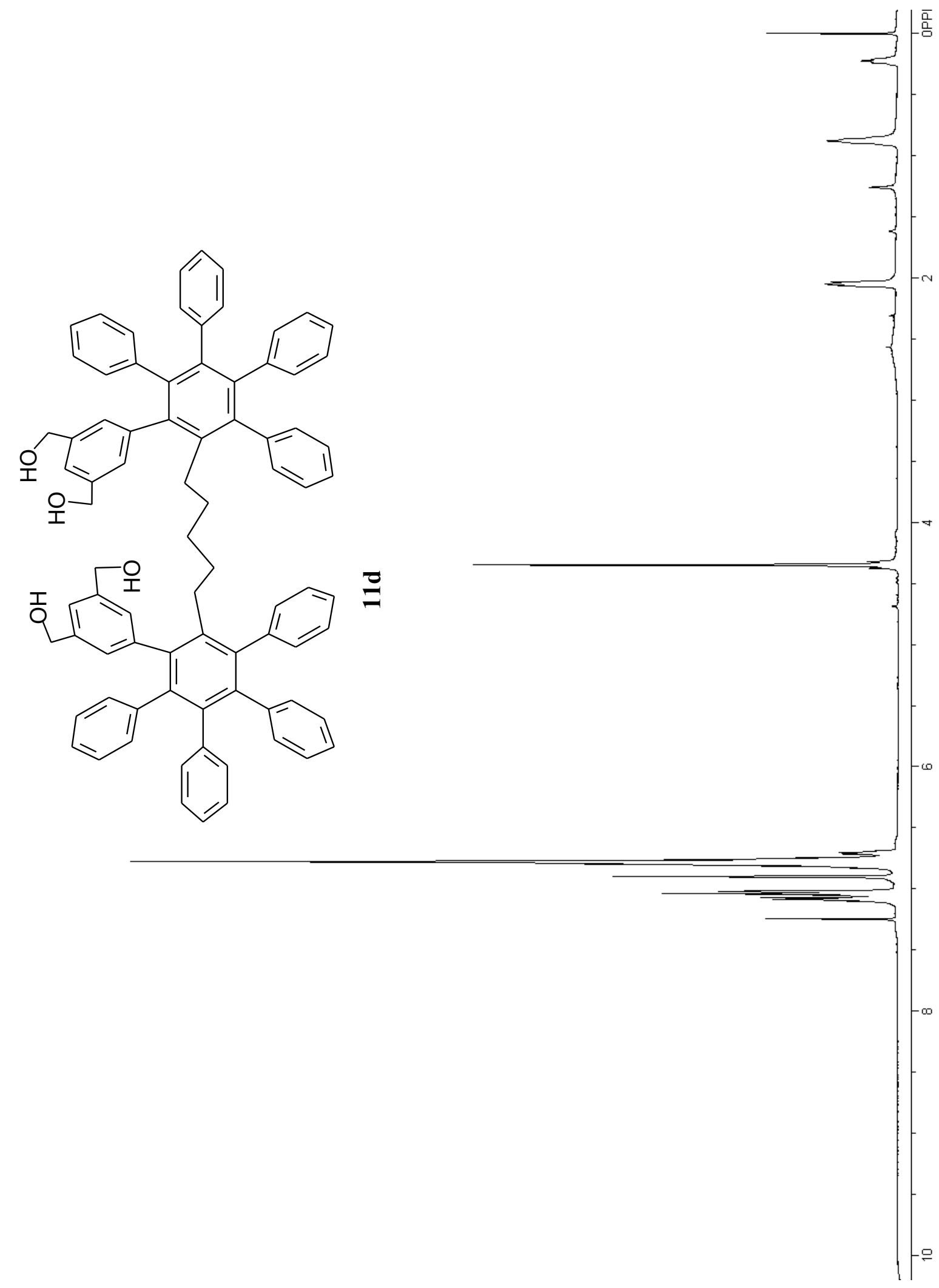




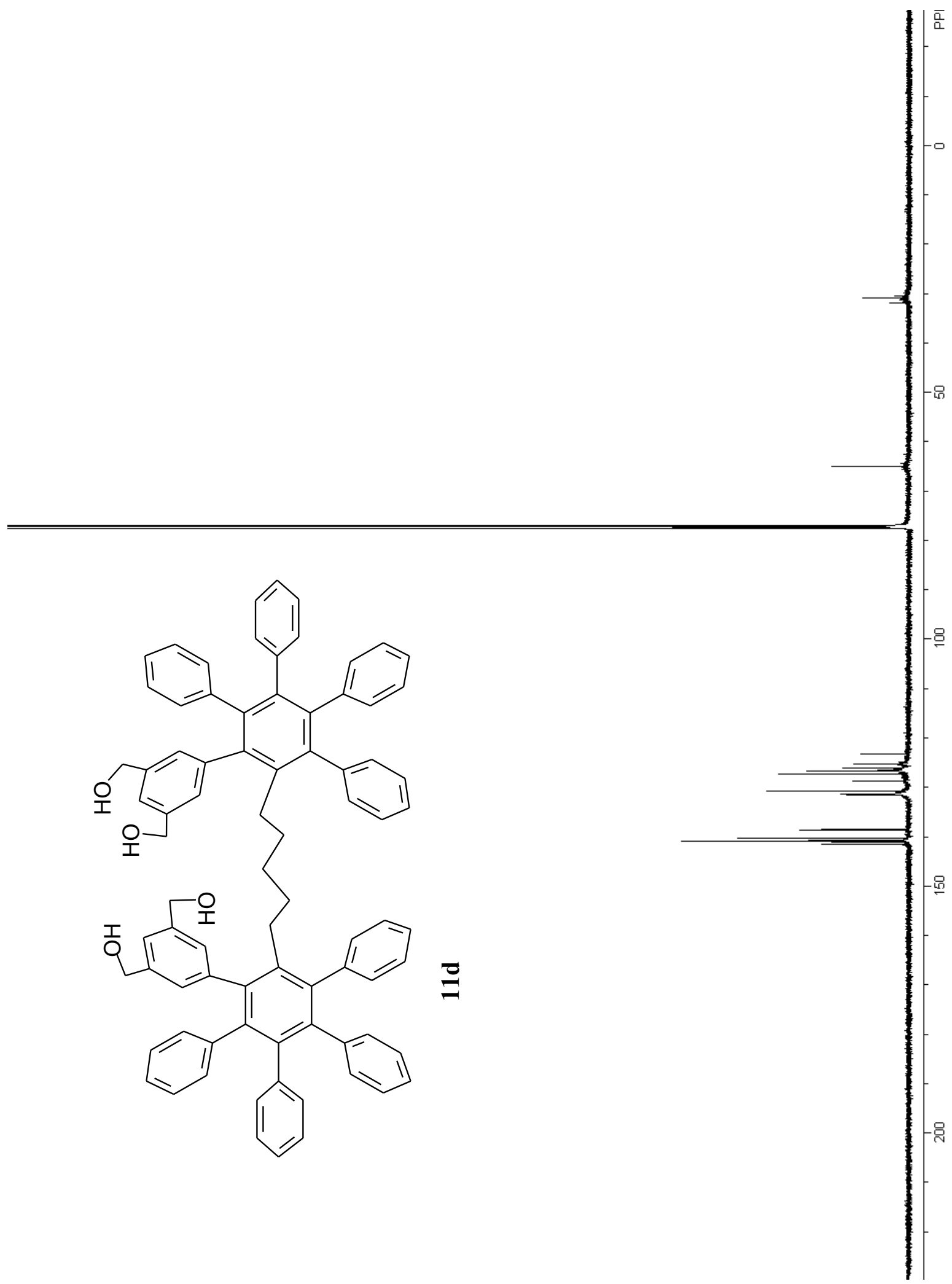

S-19 


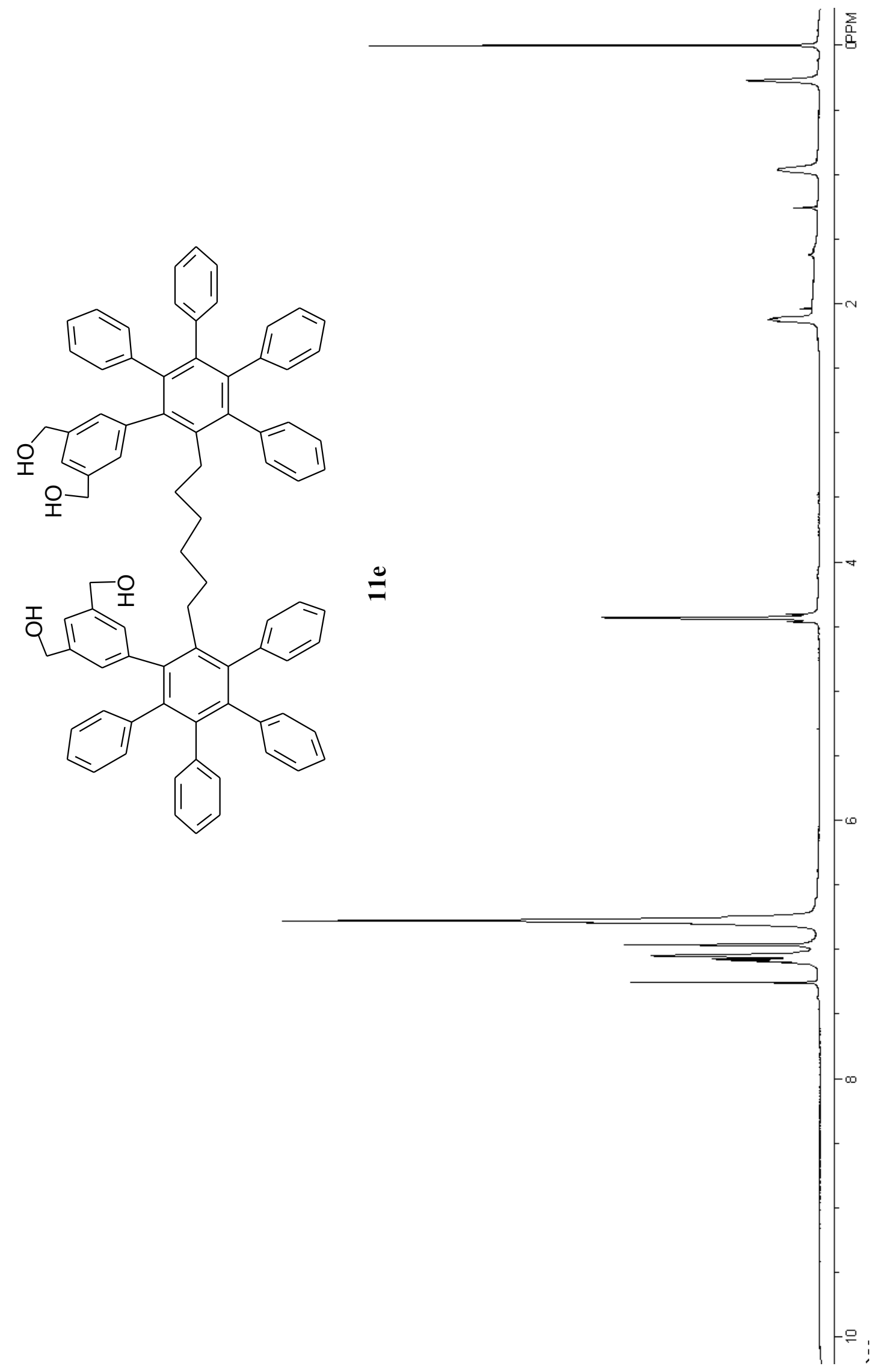




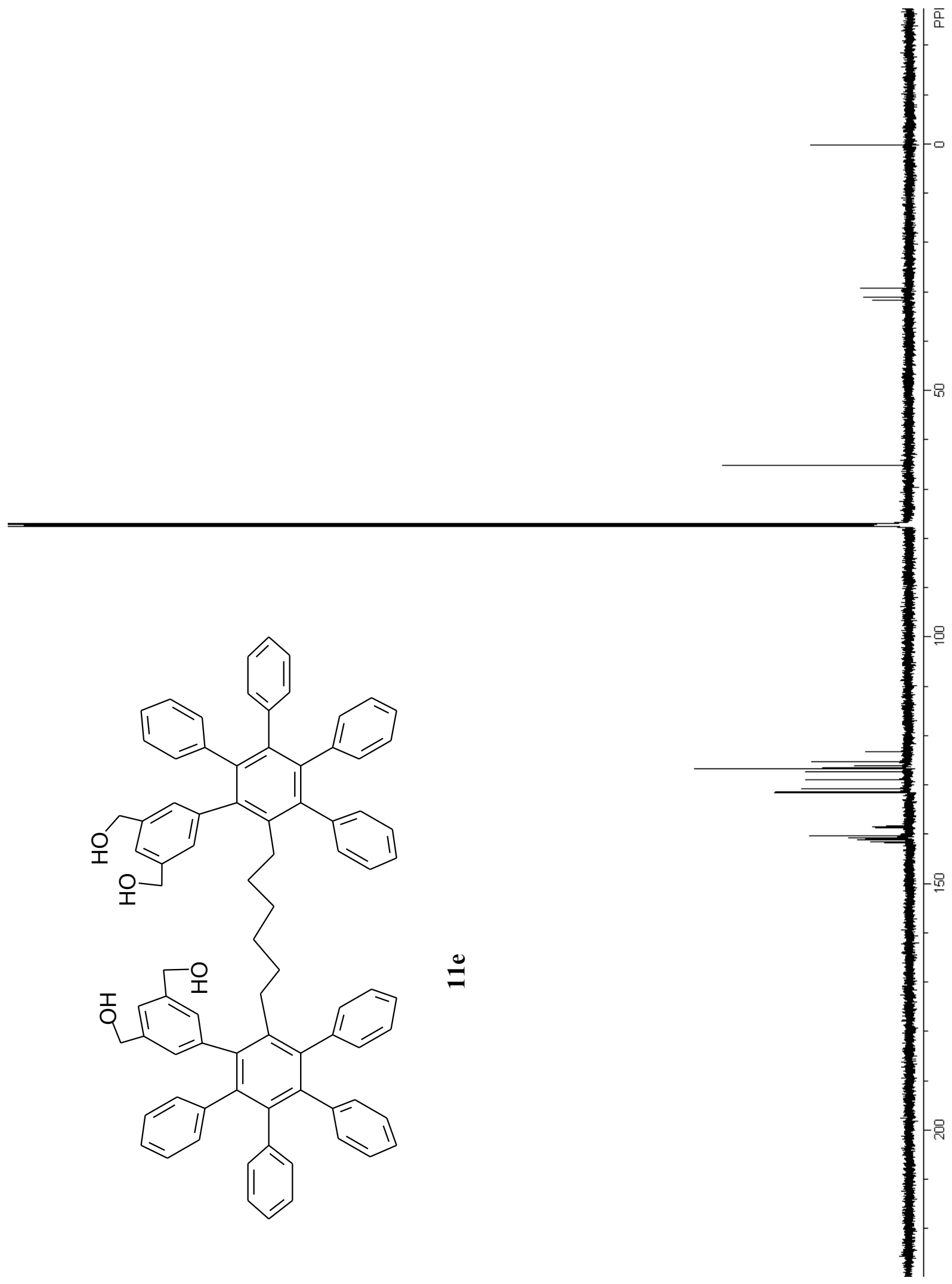



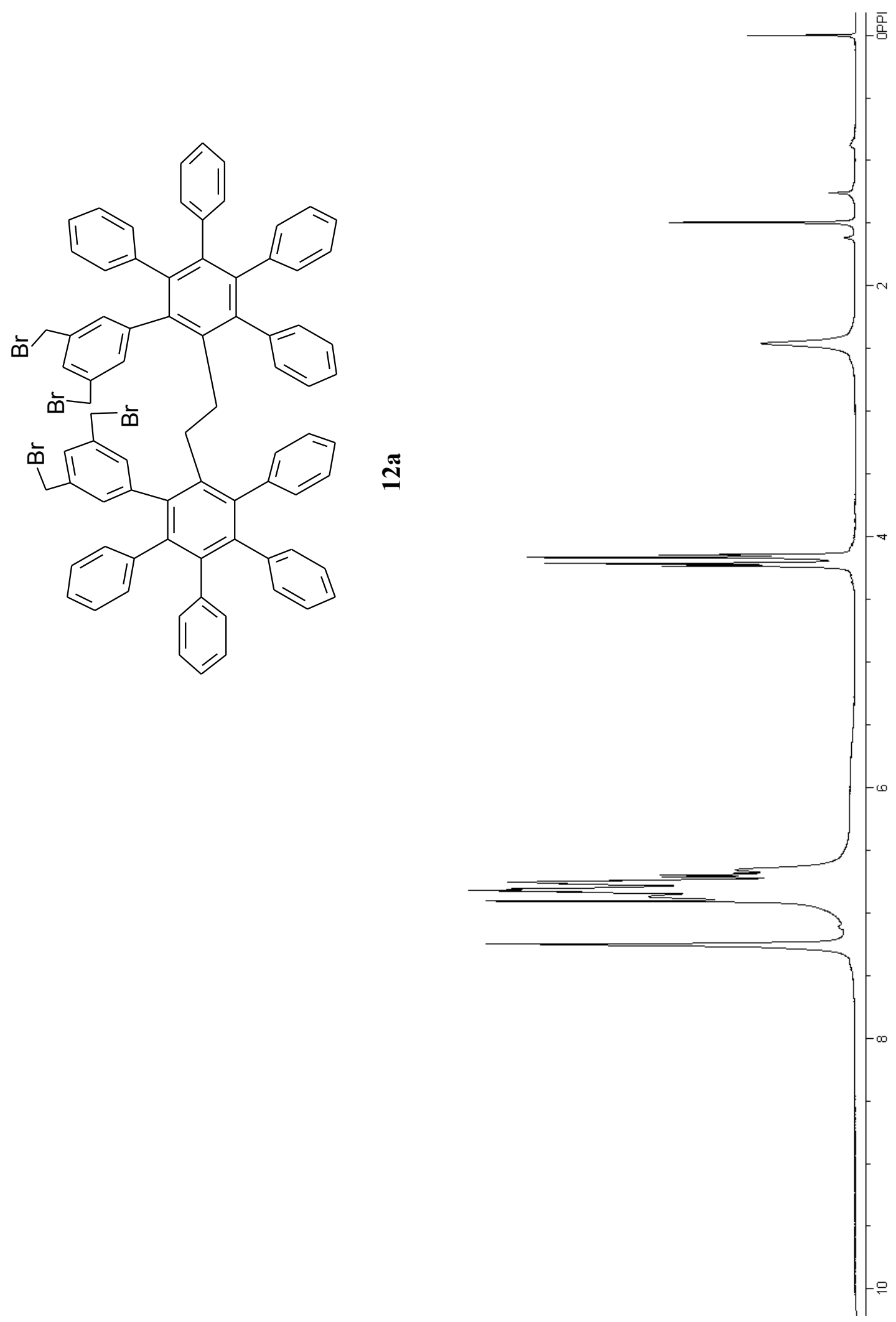

S-22 


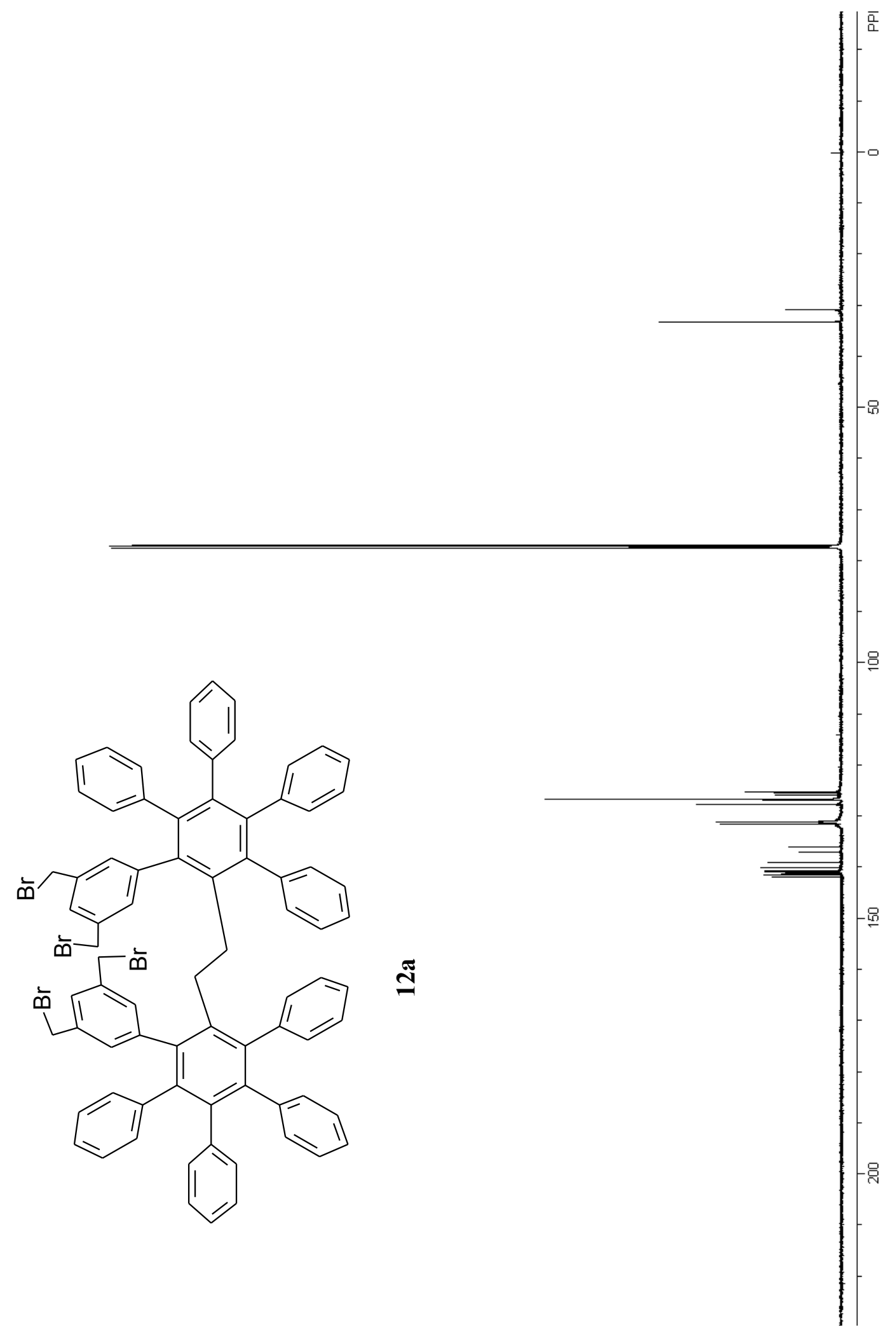




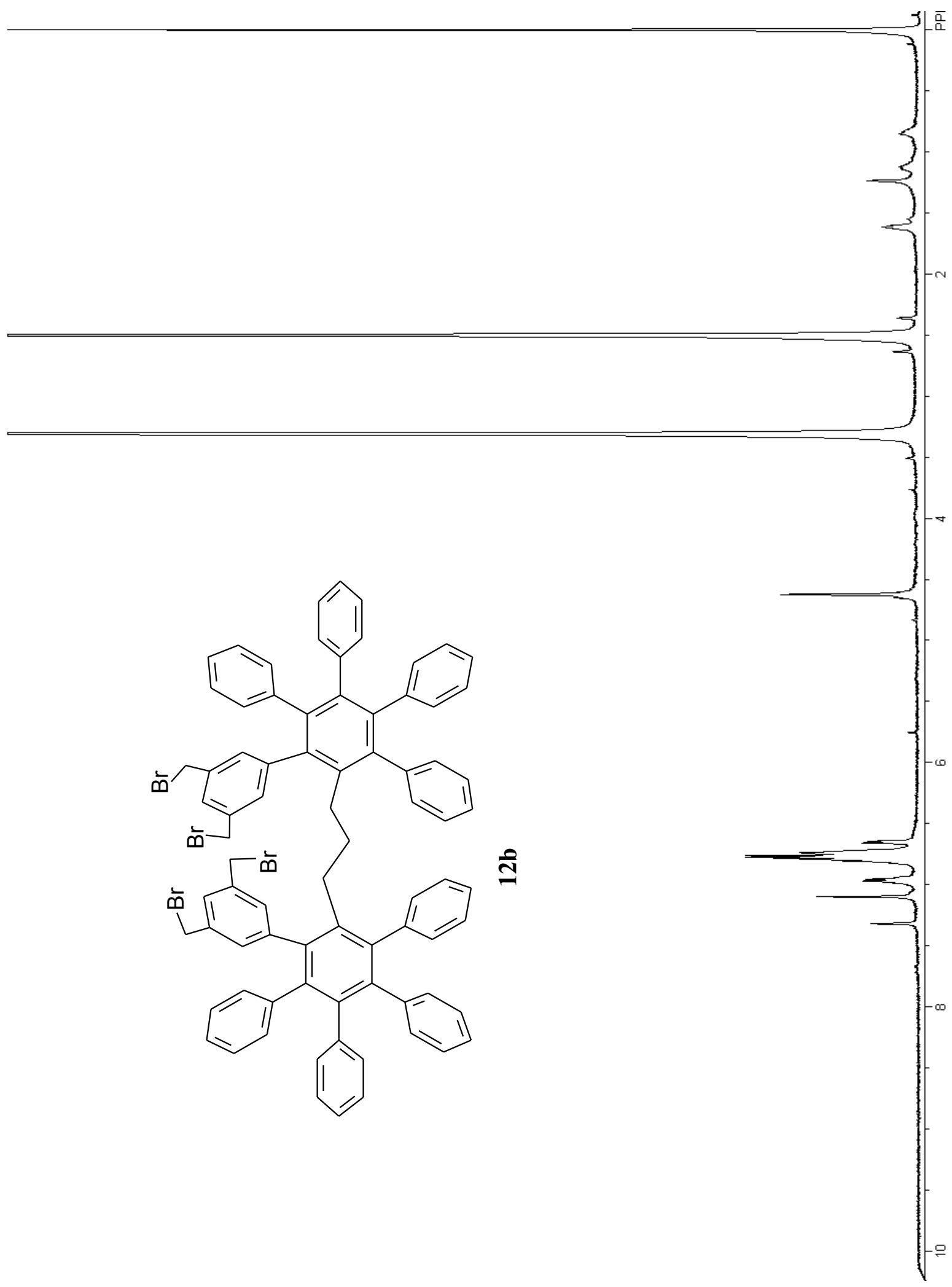




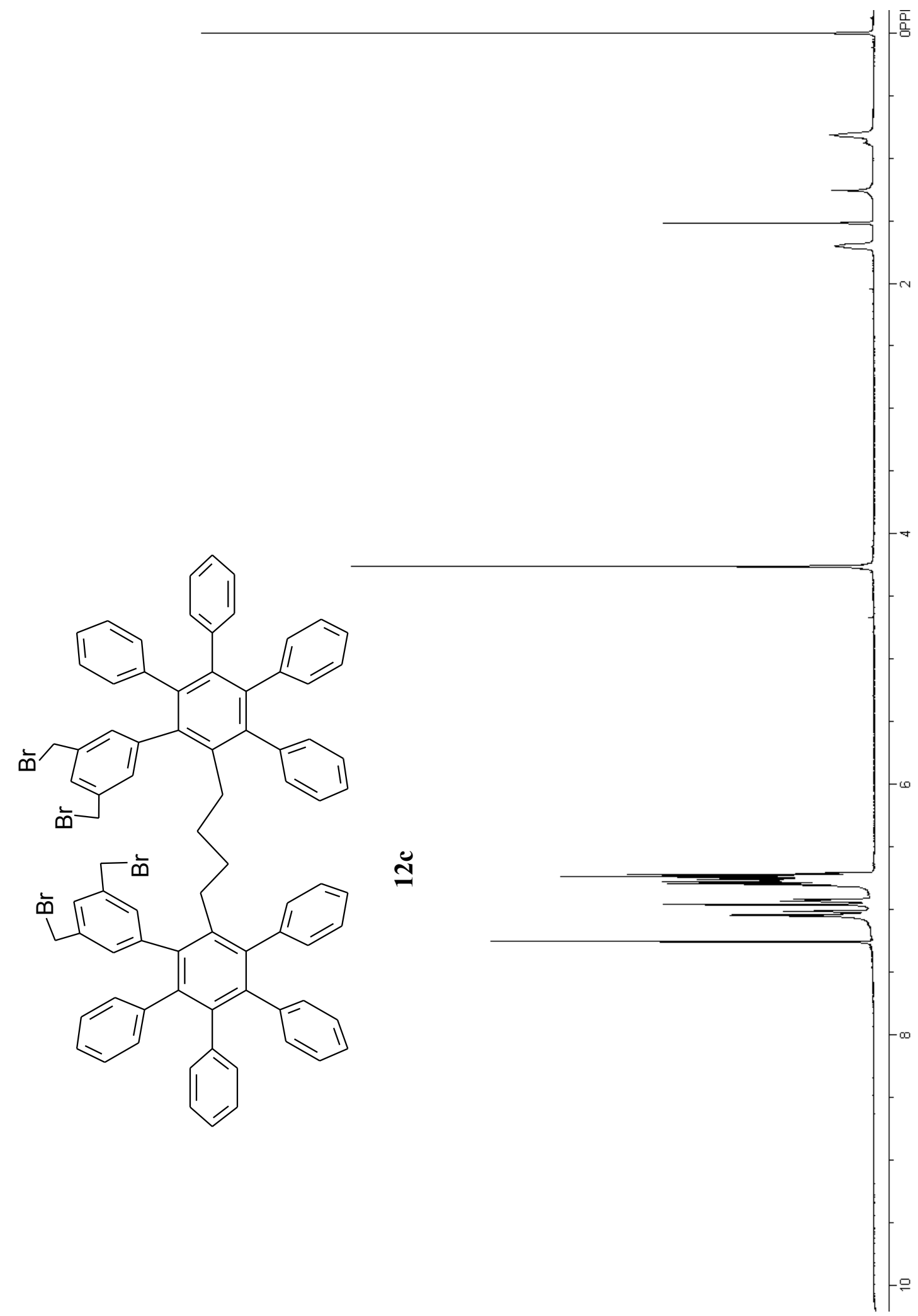




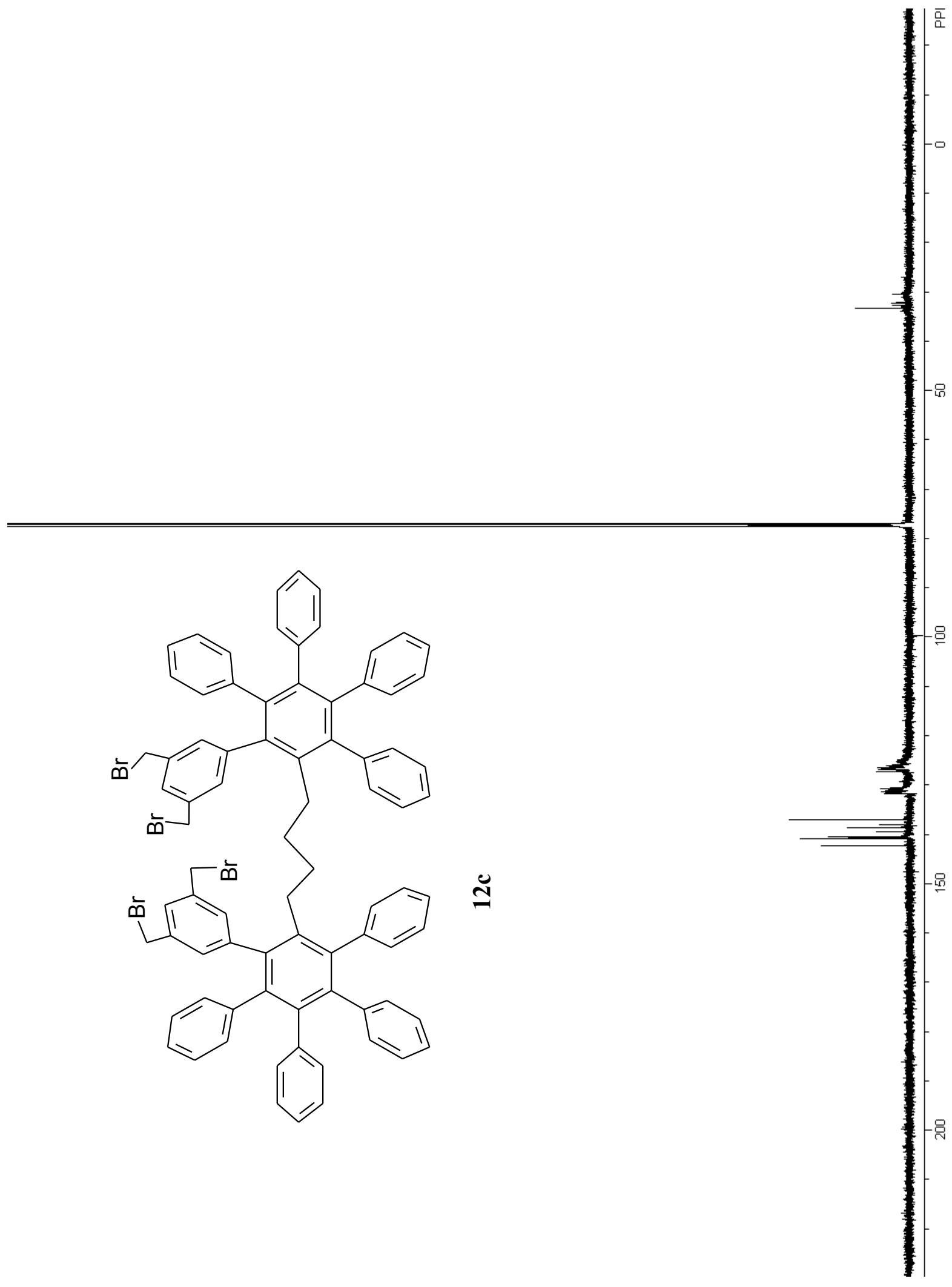




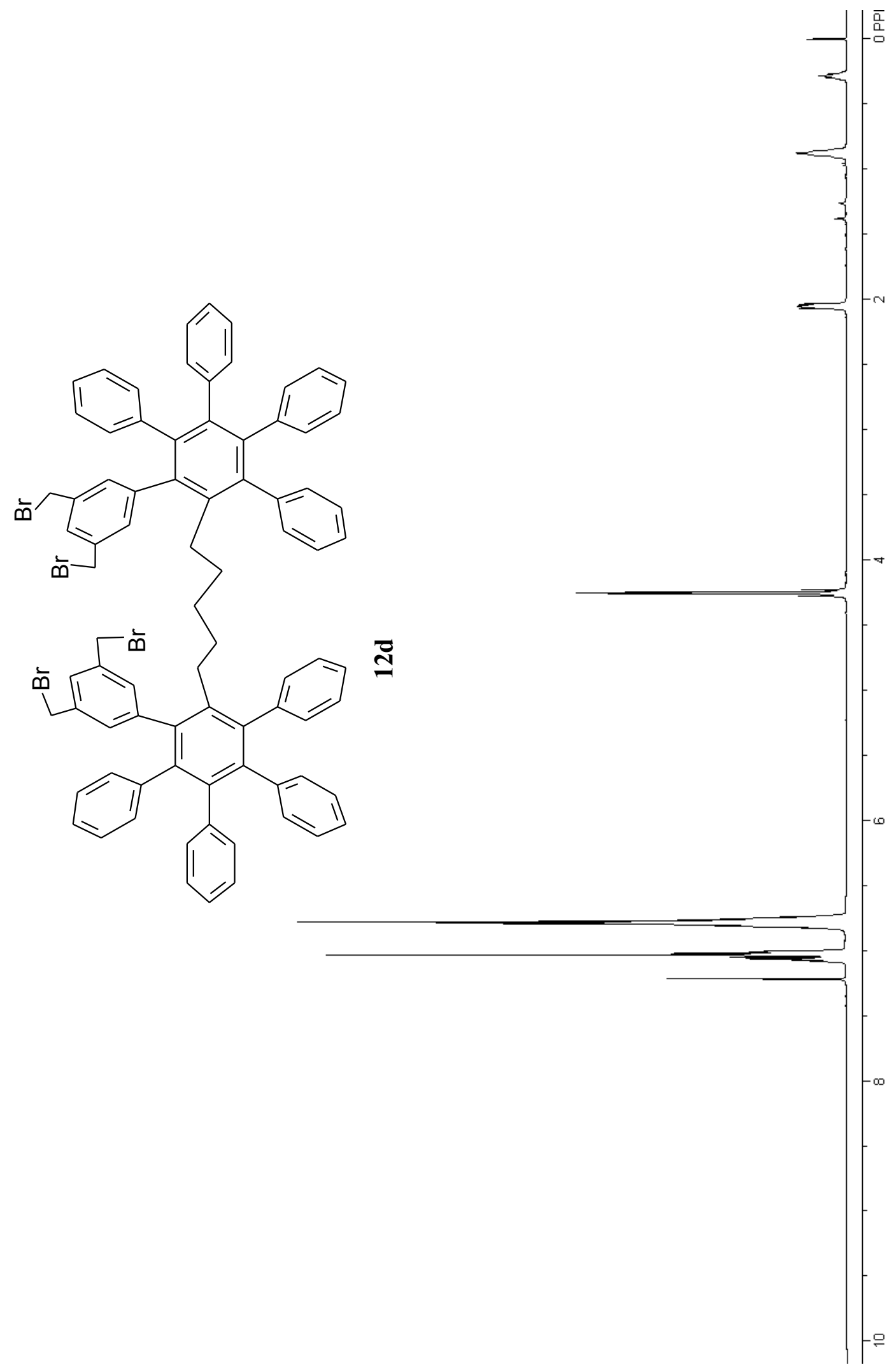




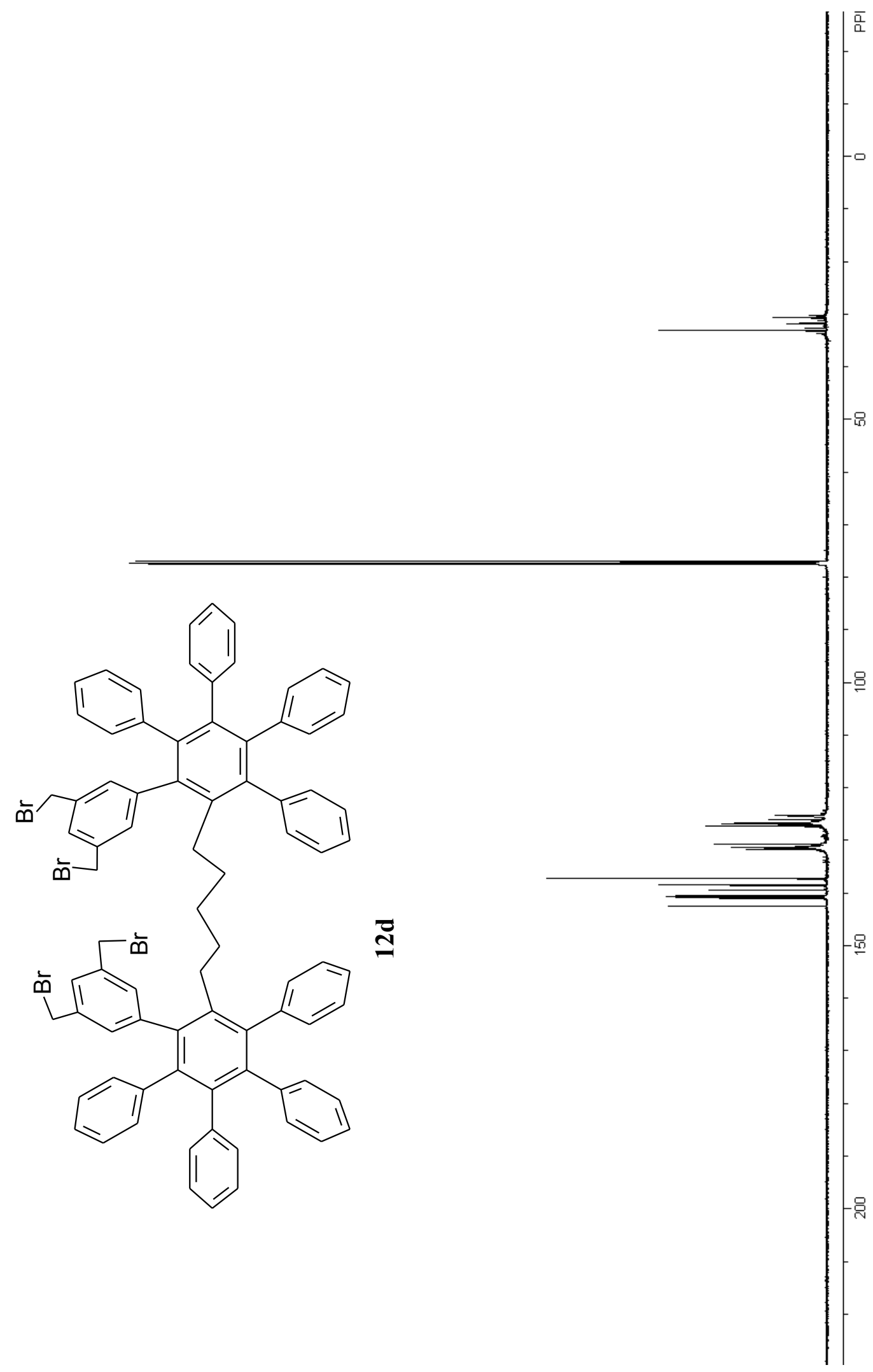




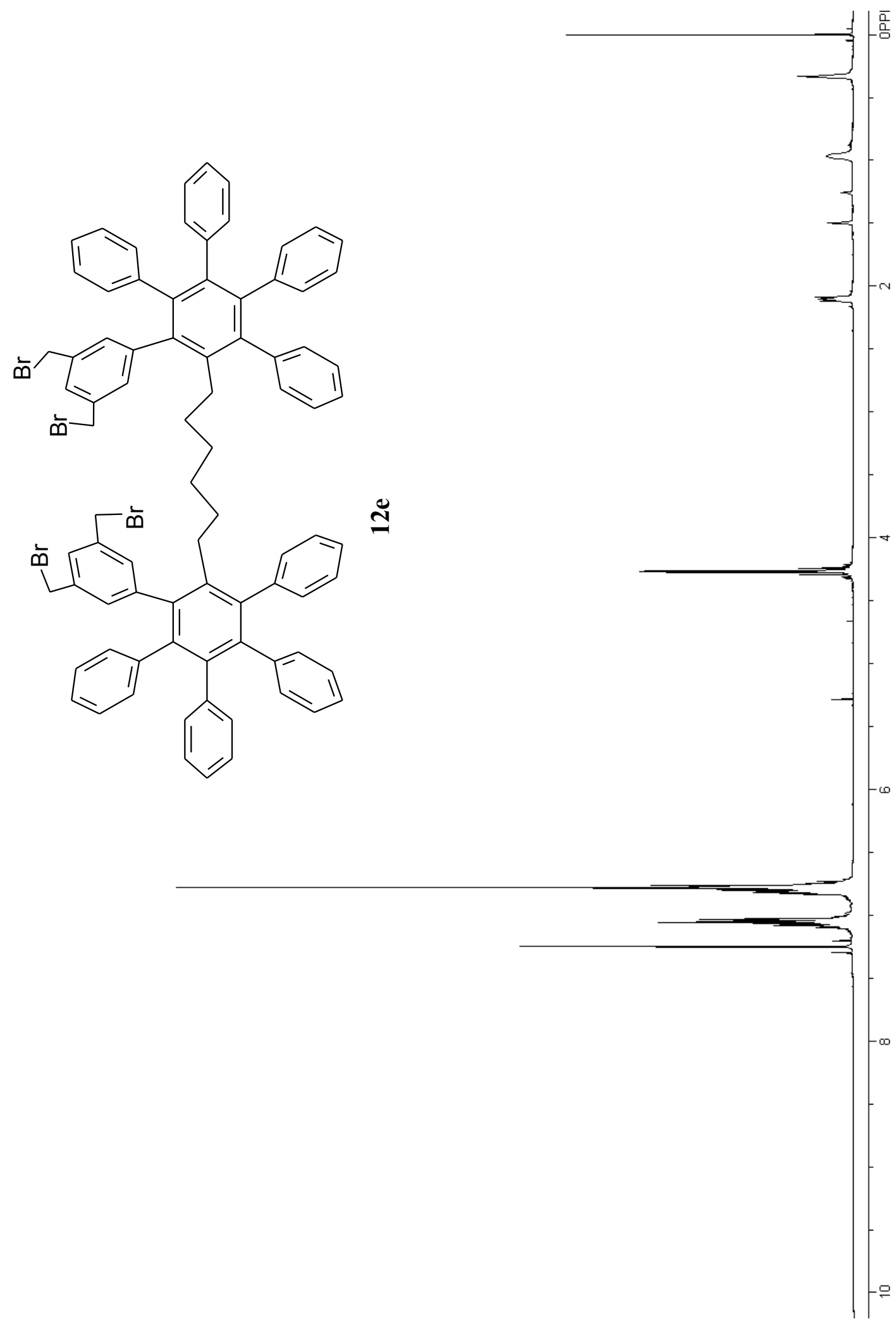




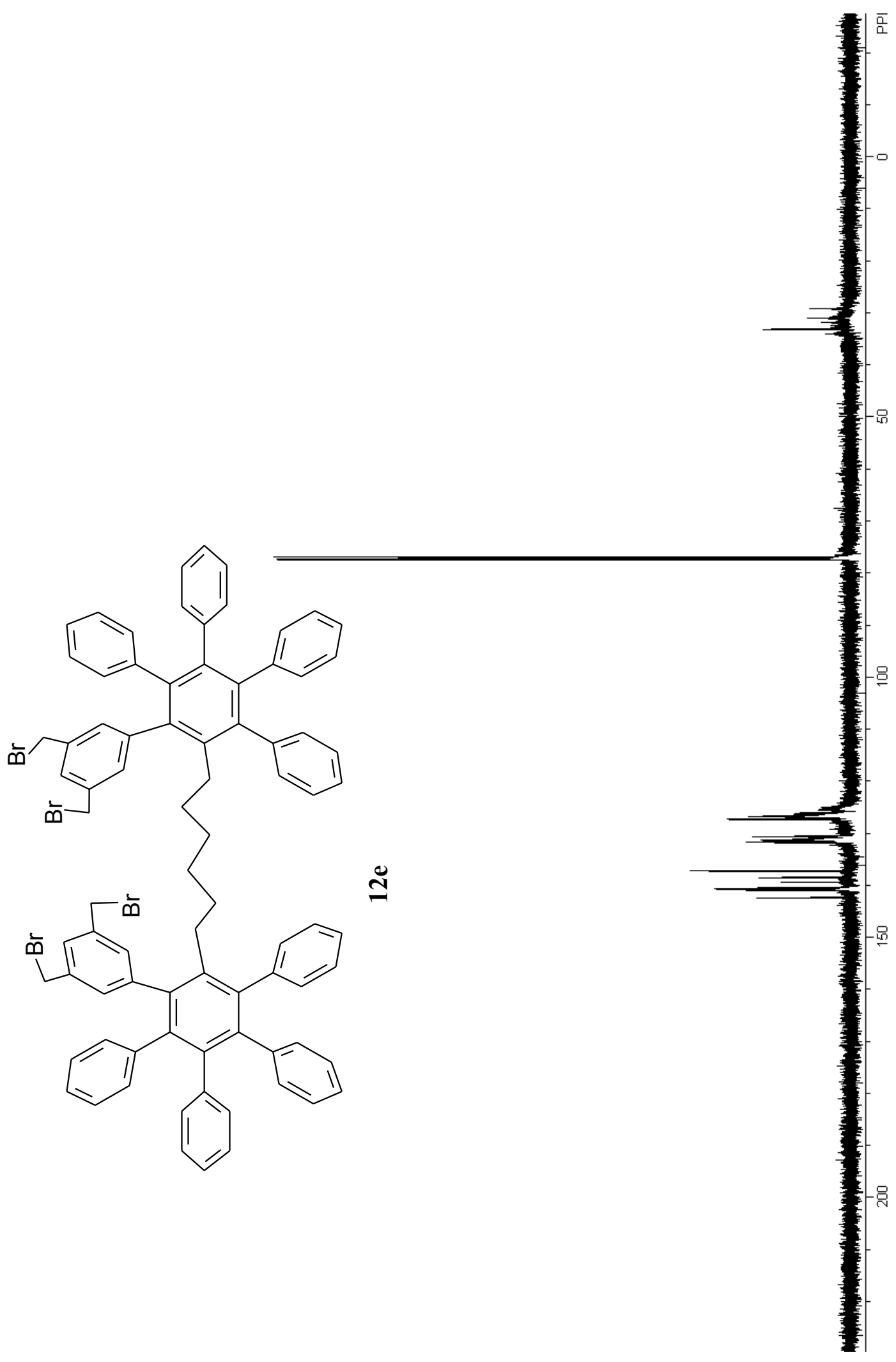

S-30 


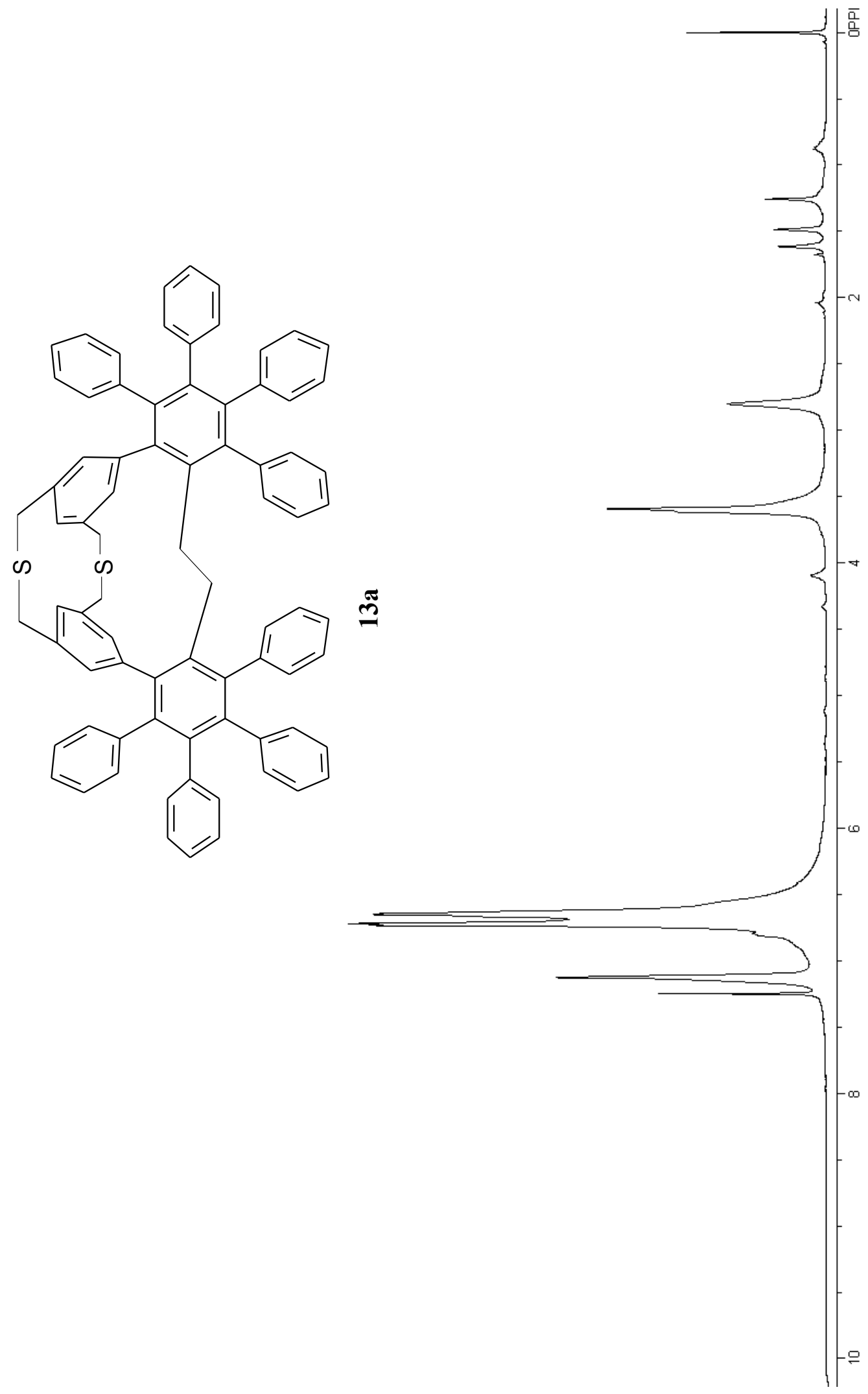

S-31 


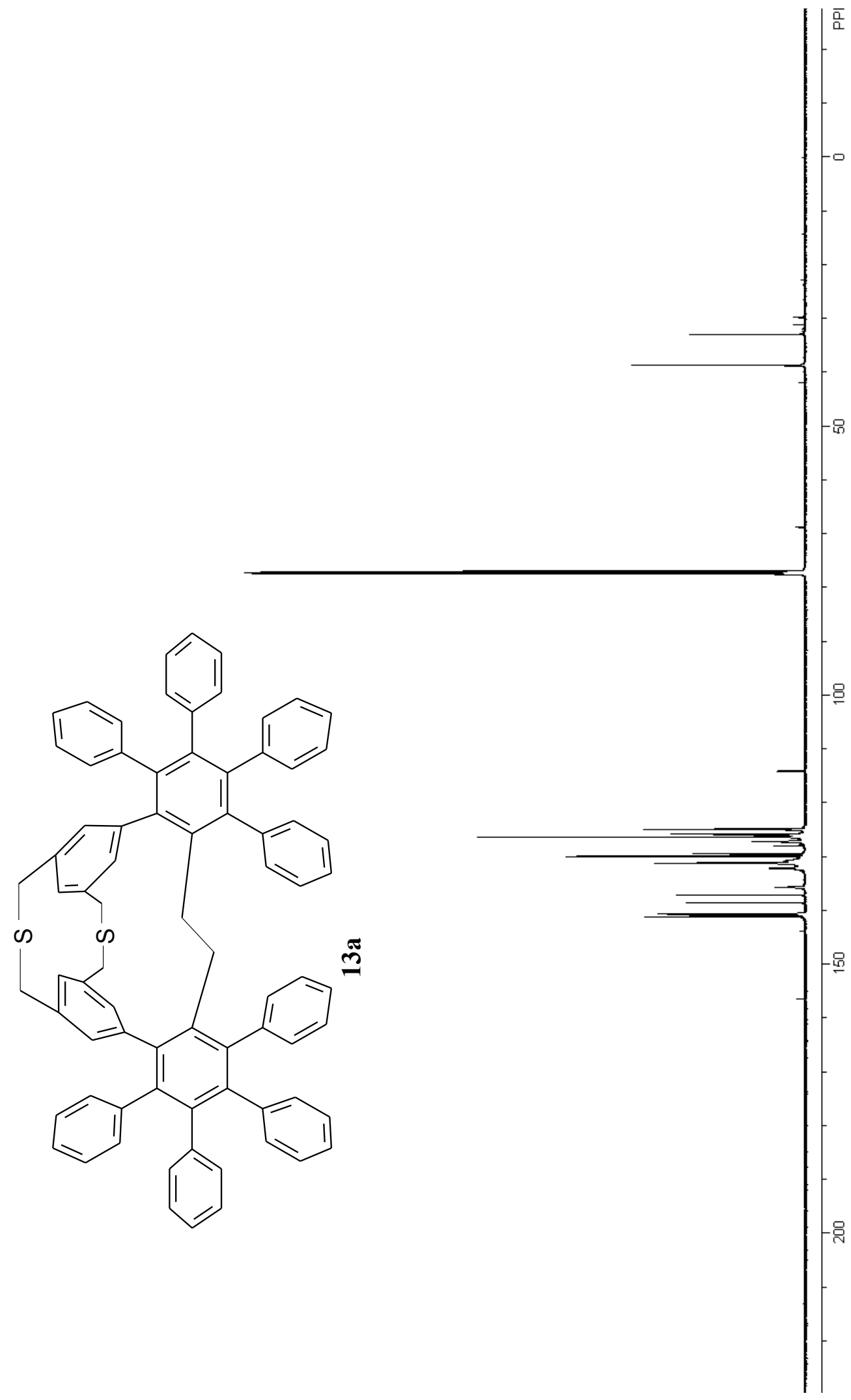




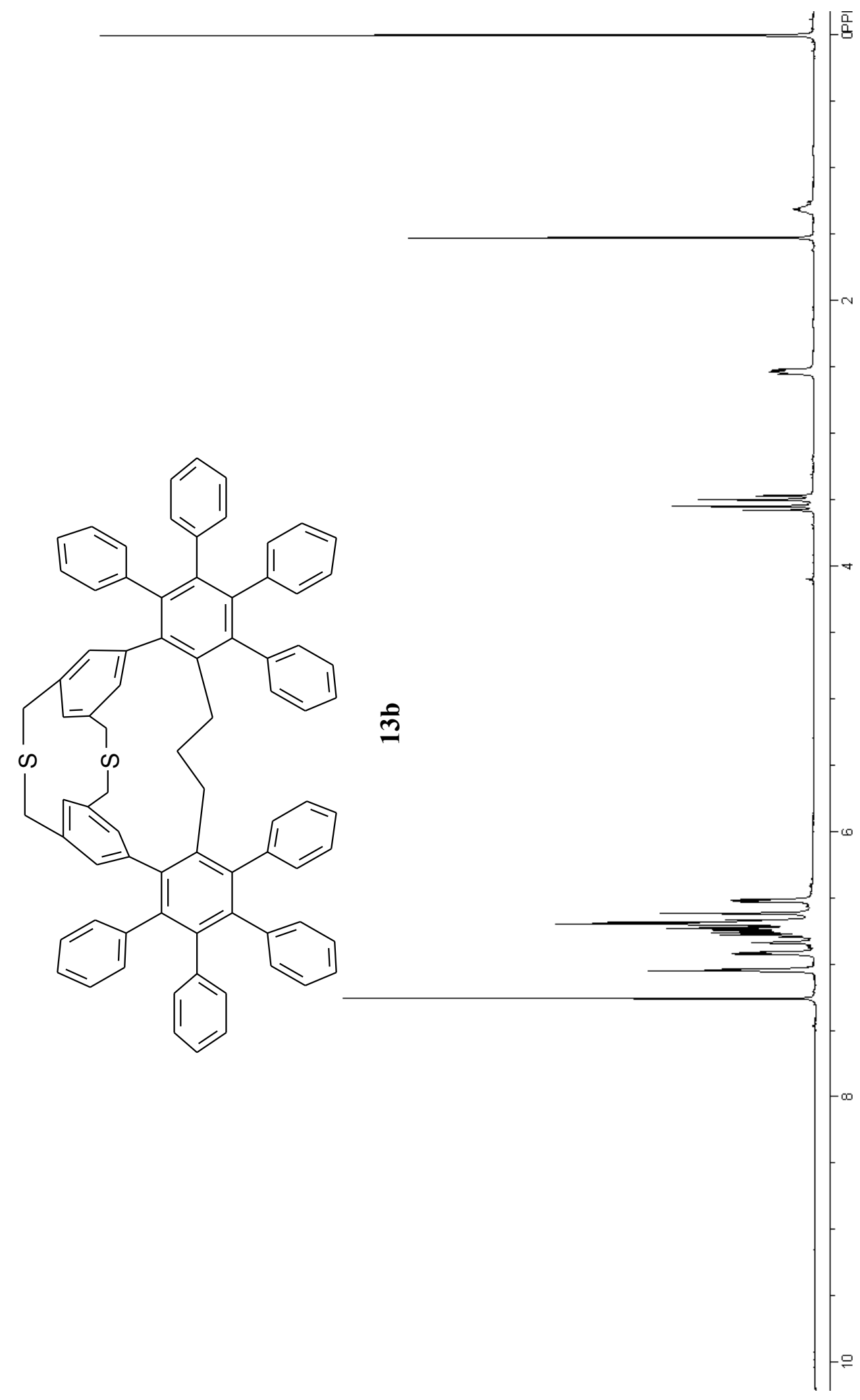

S-33 


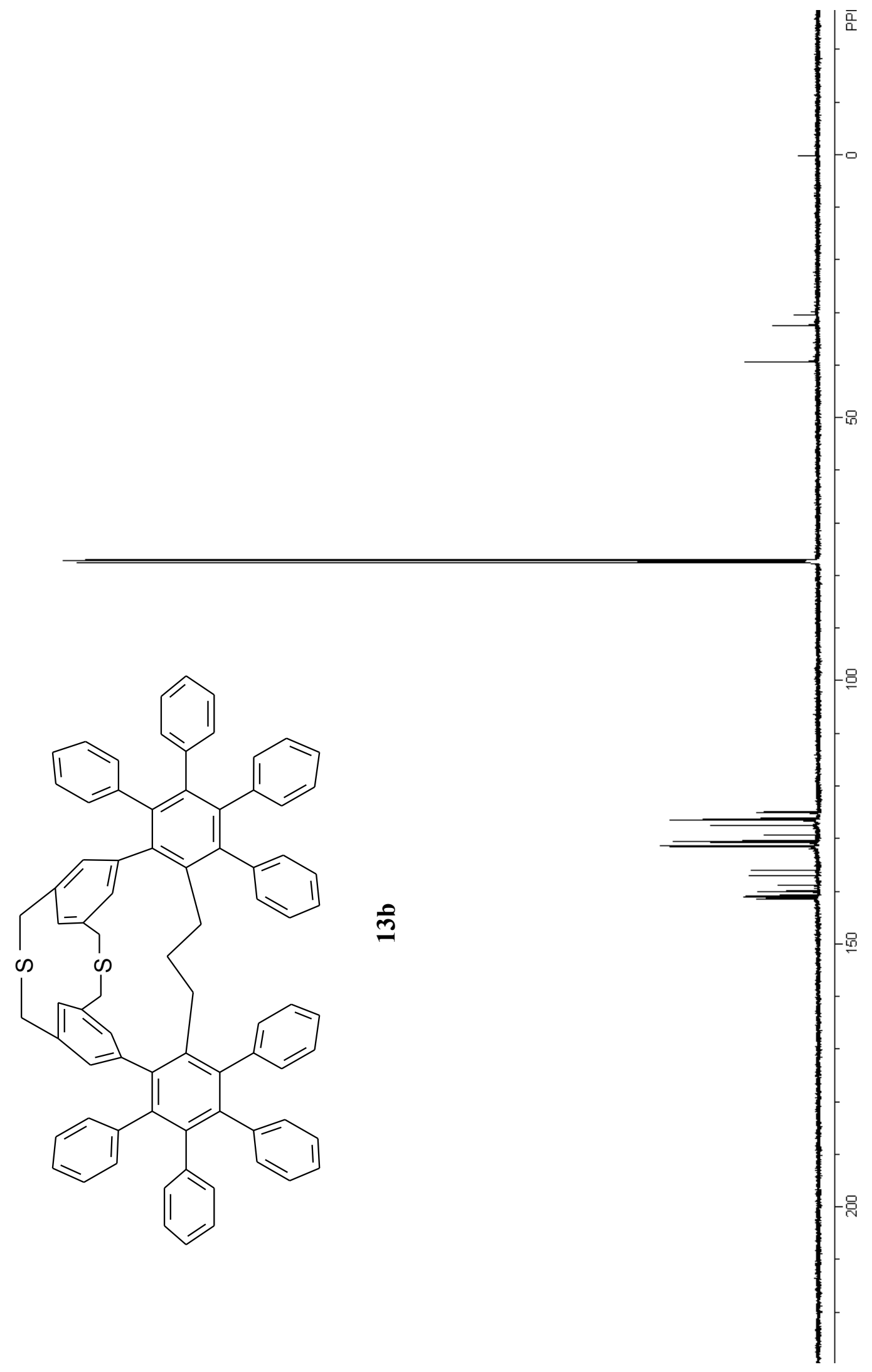




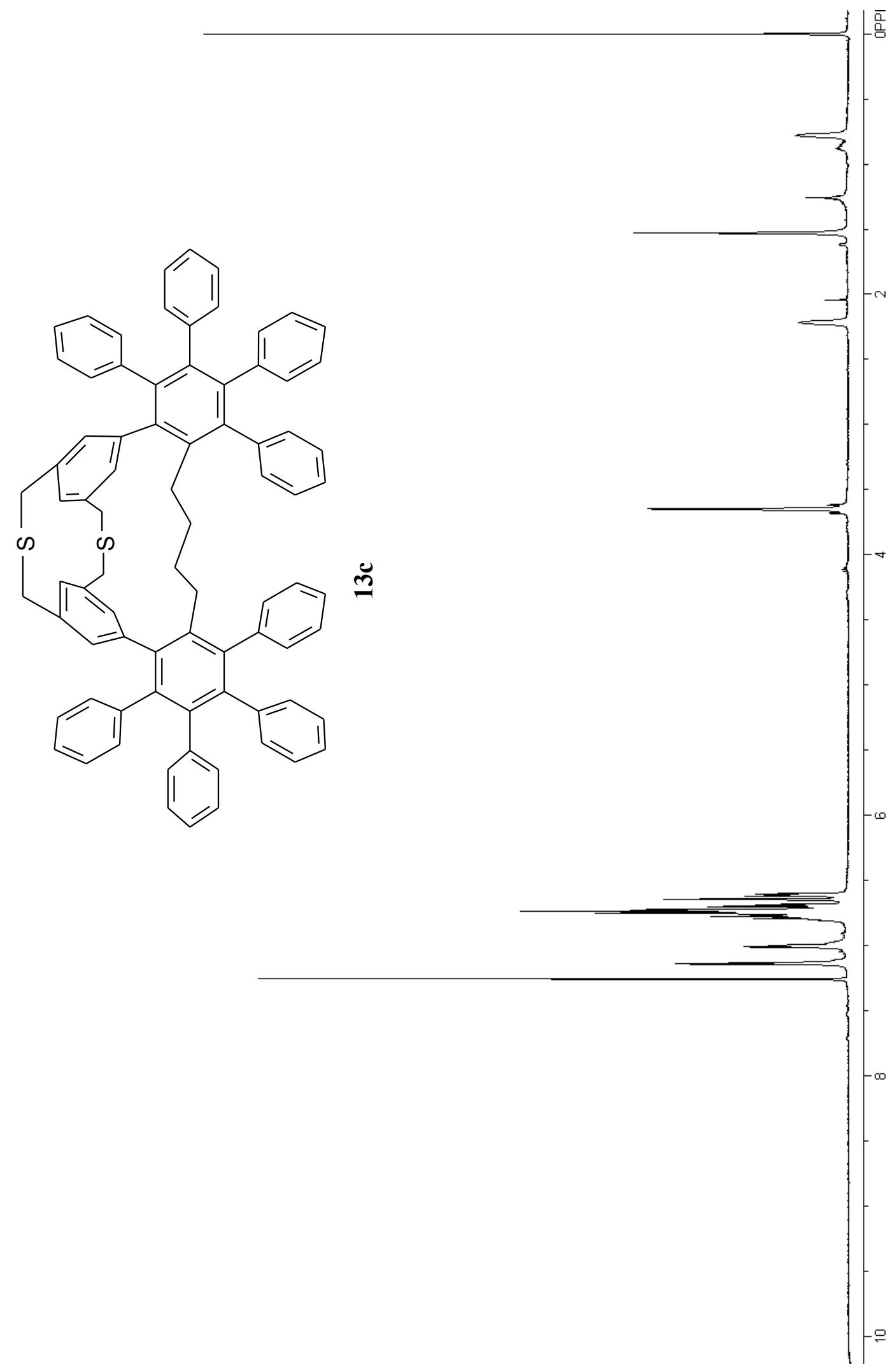



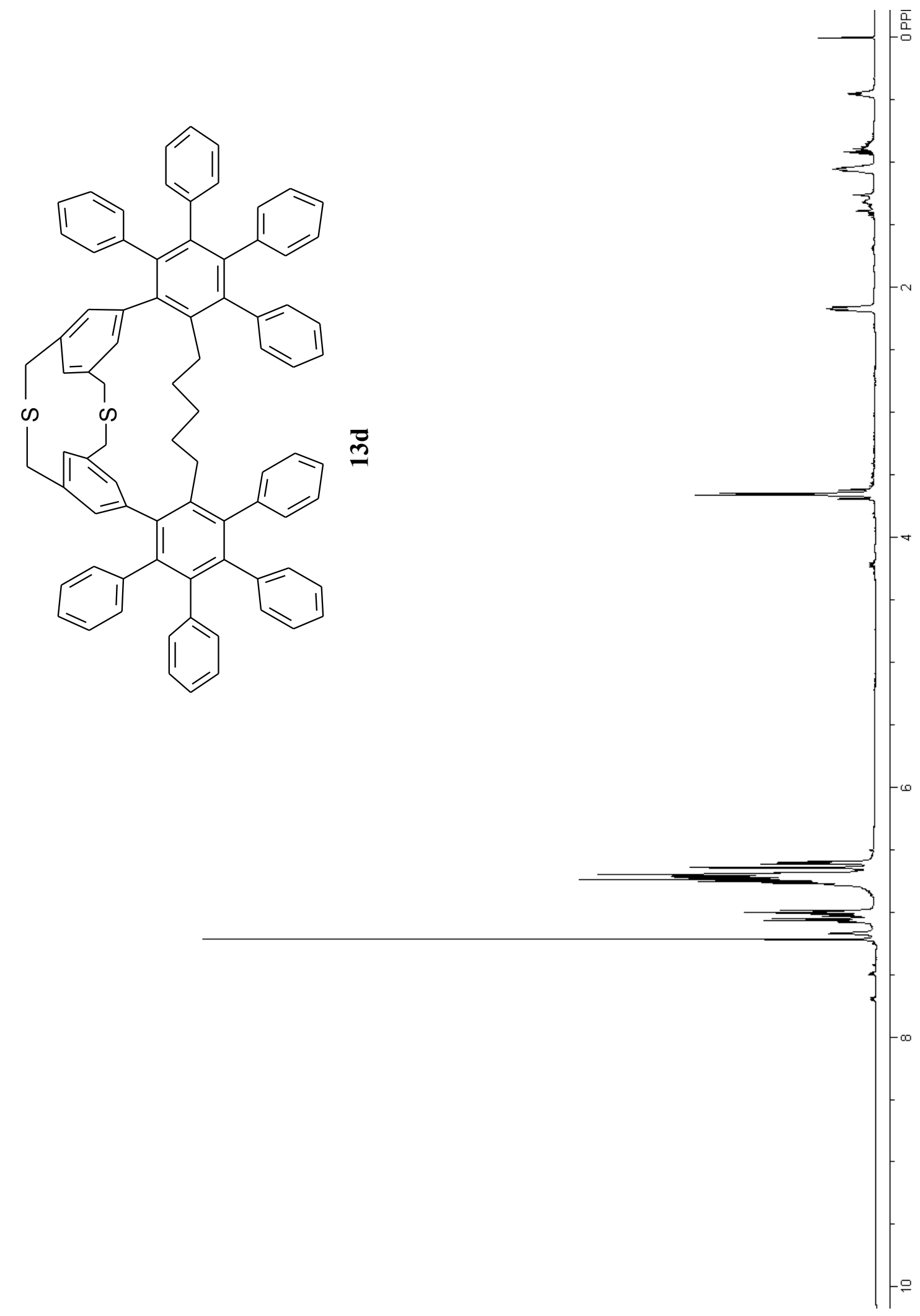


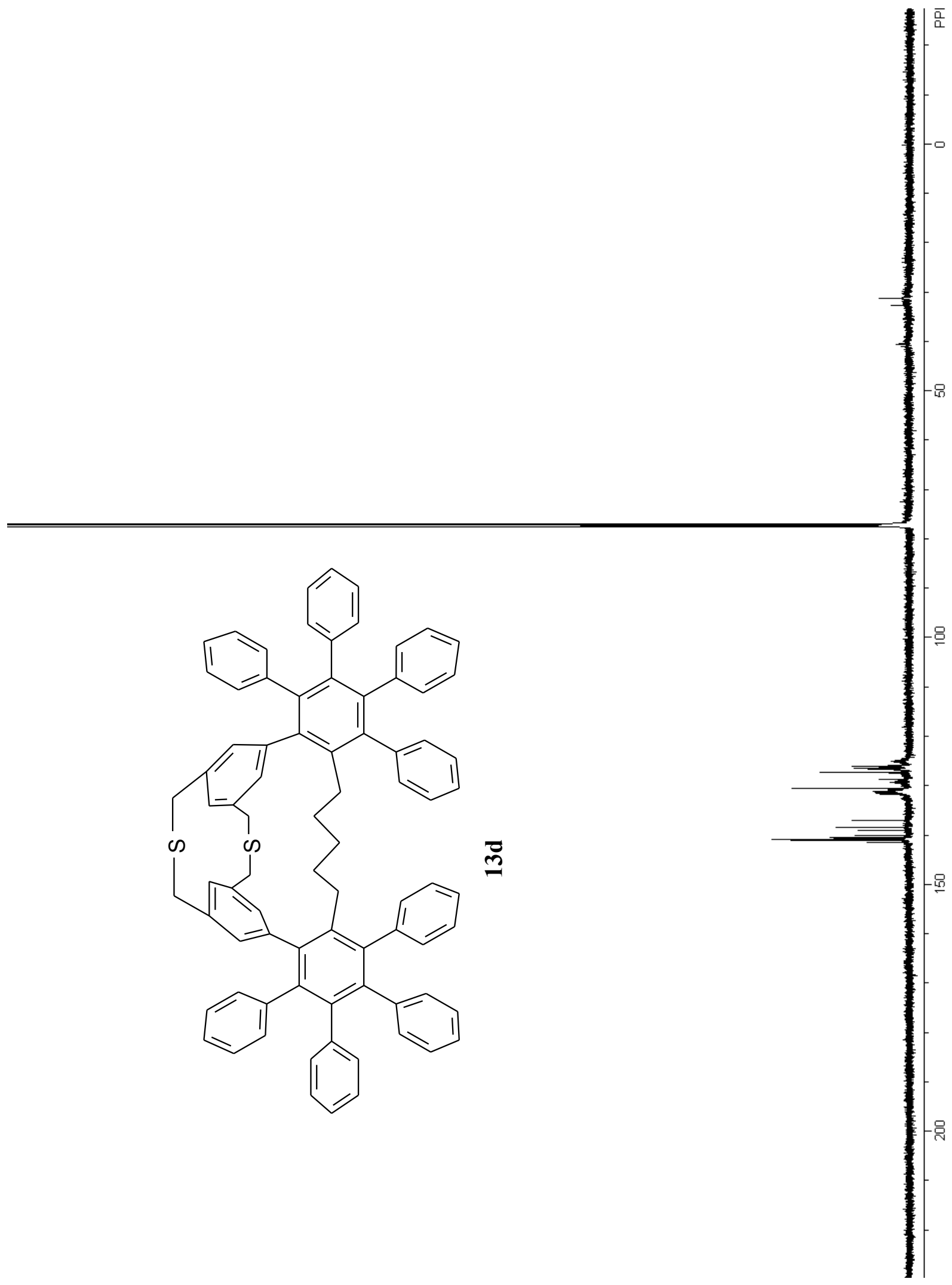




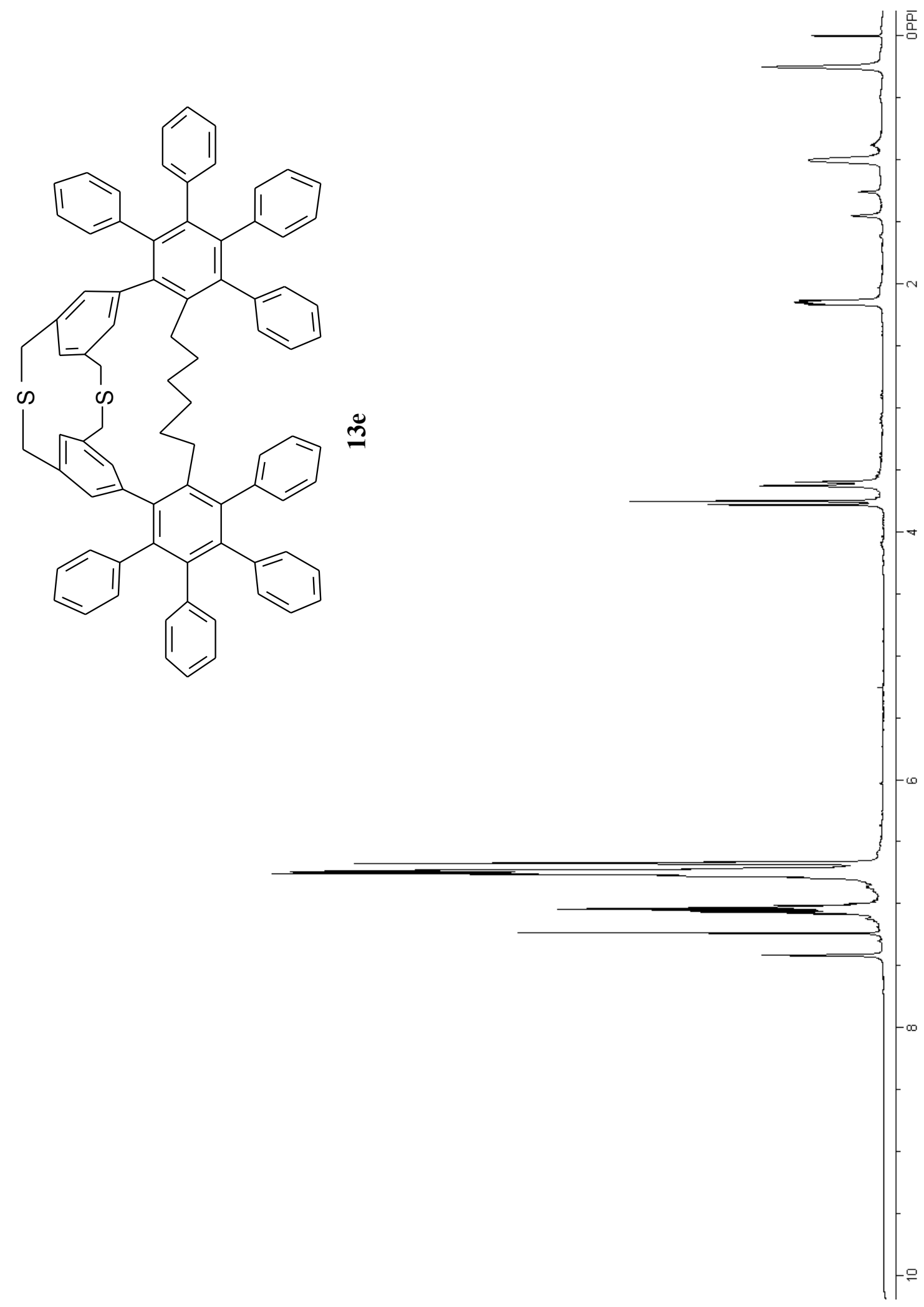




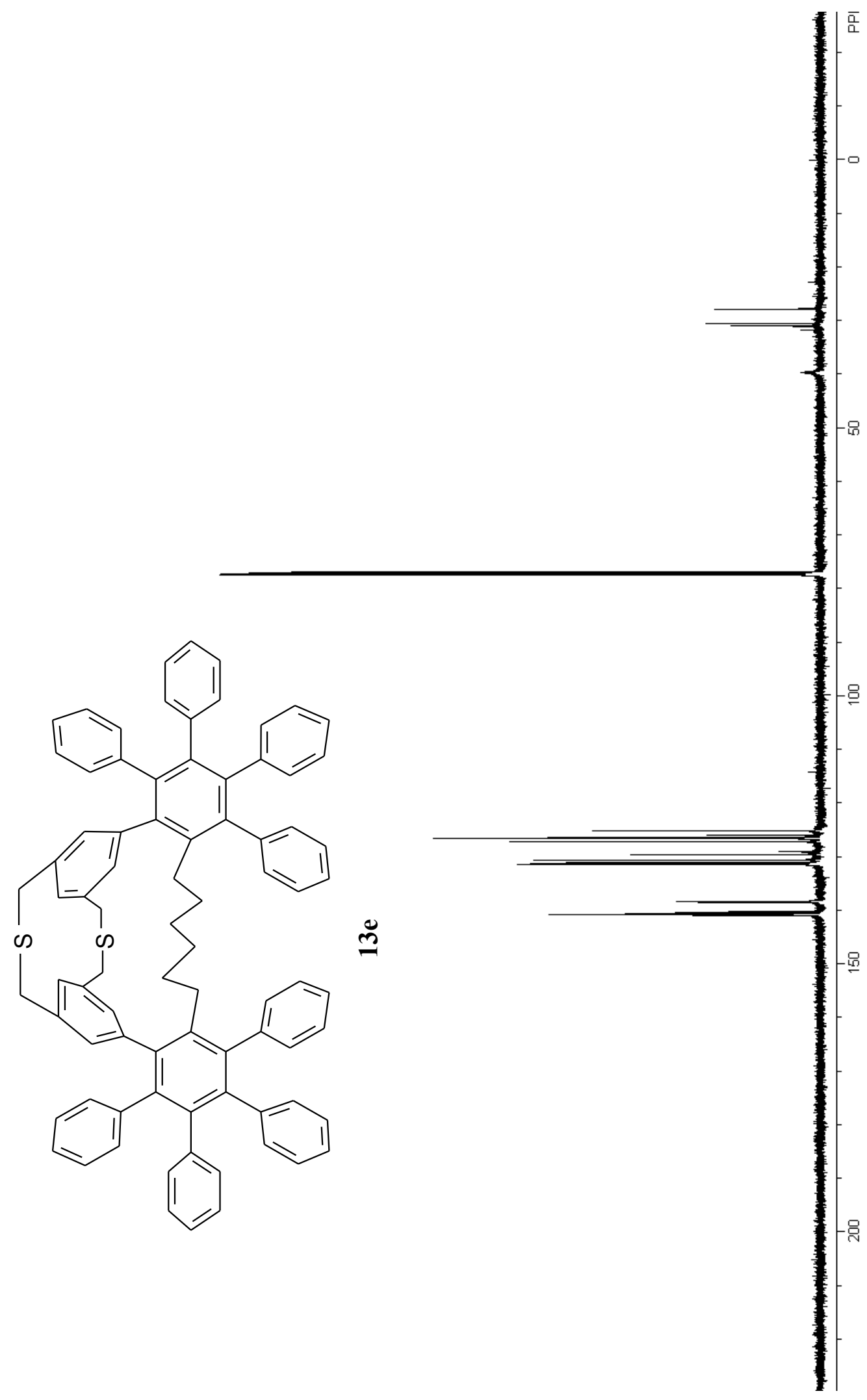

S-39 


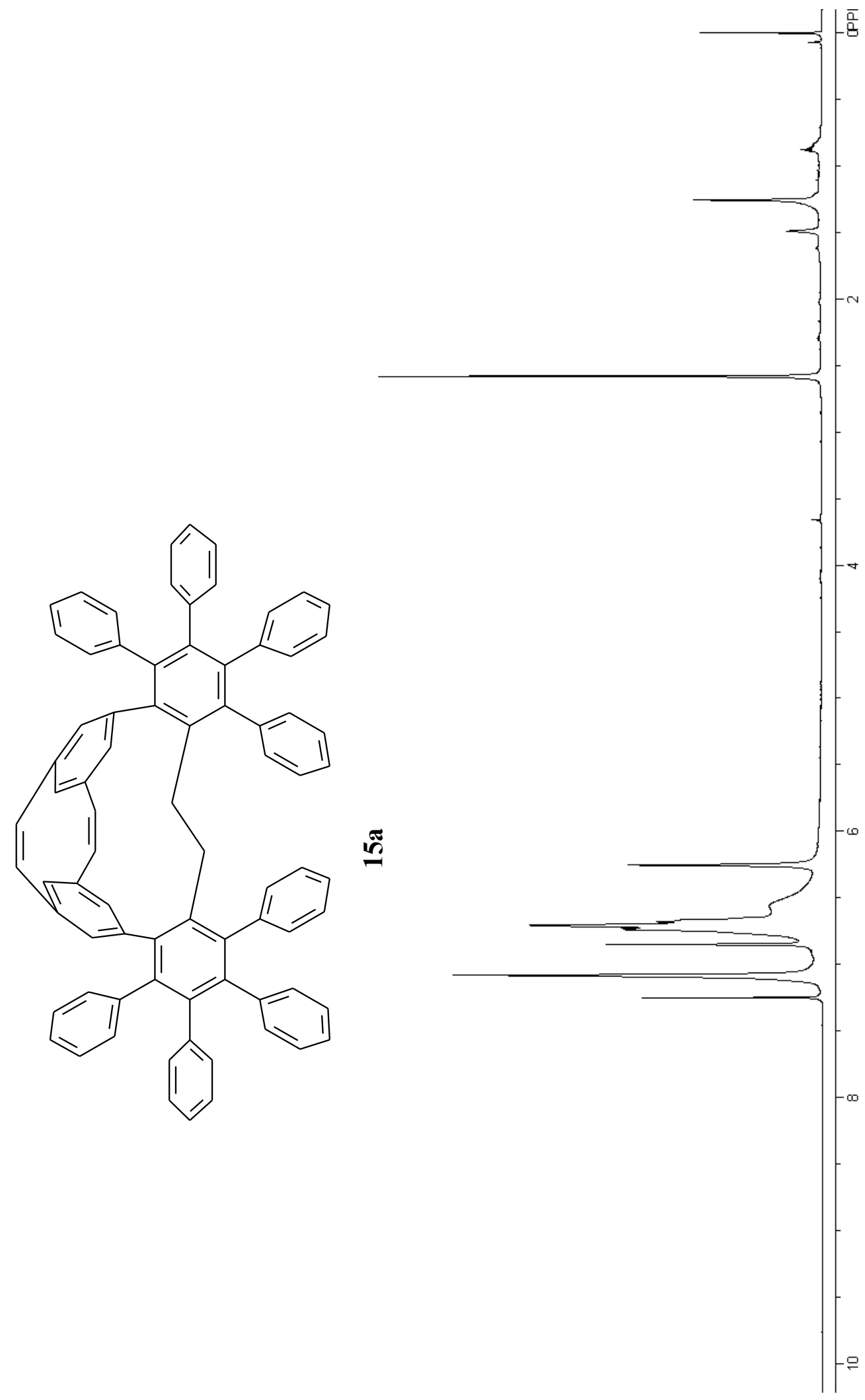




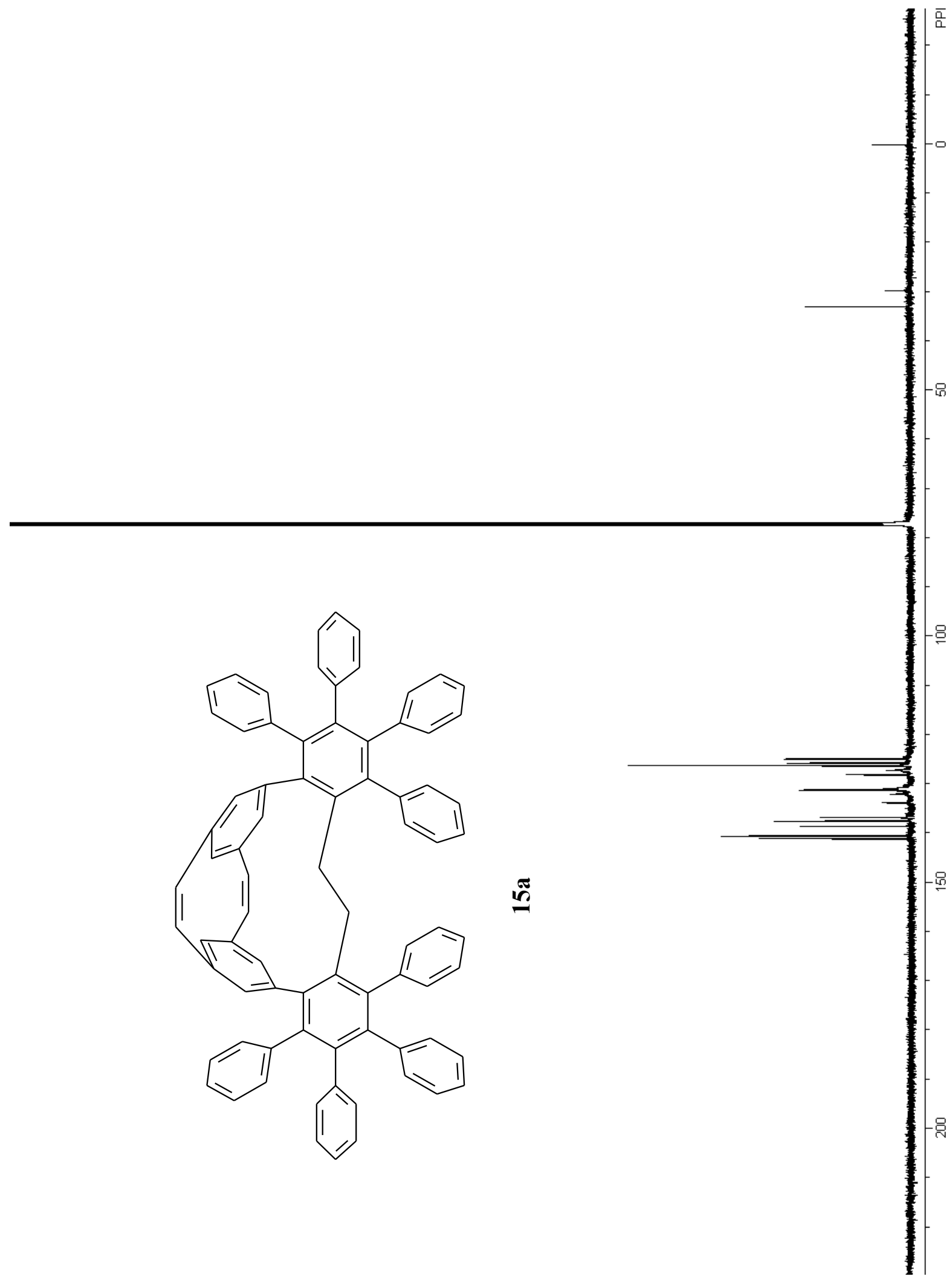




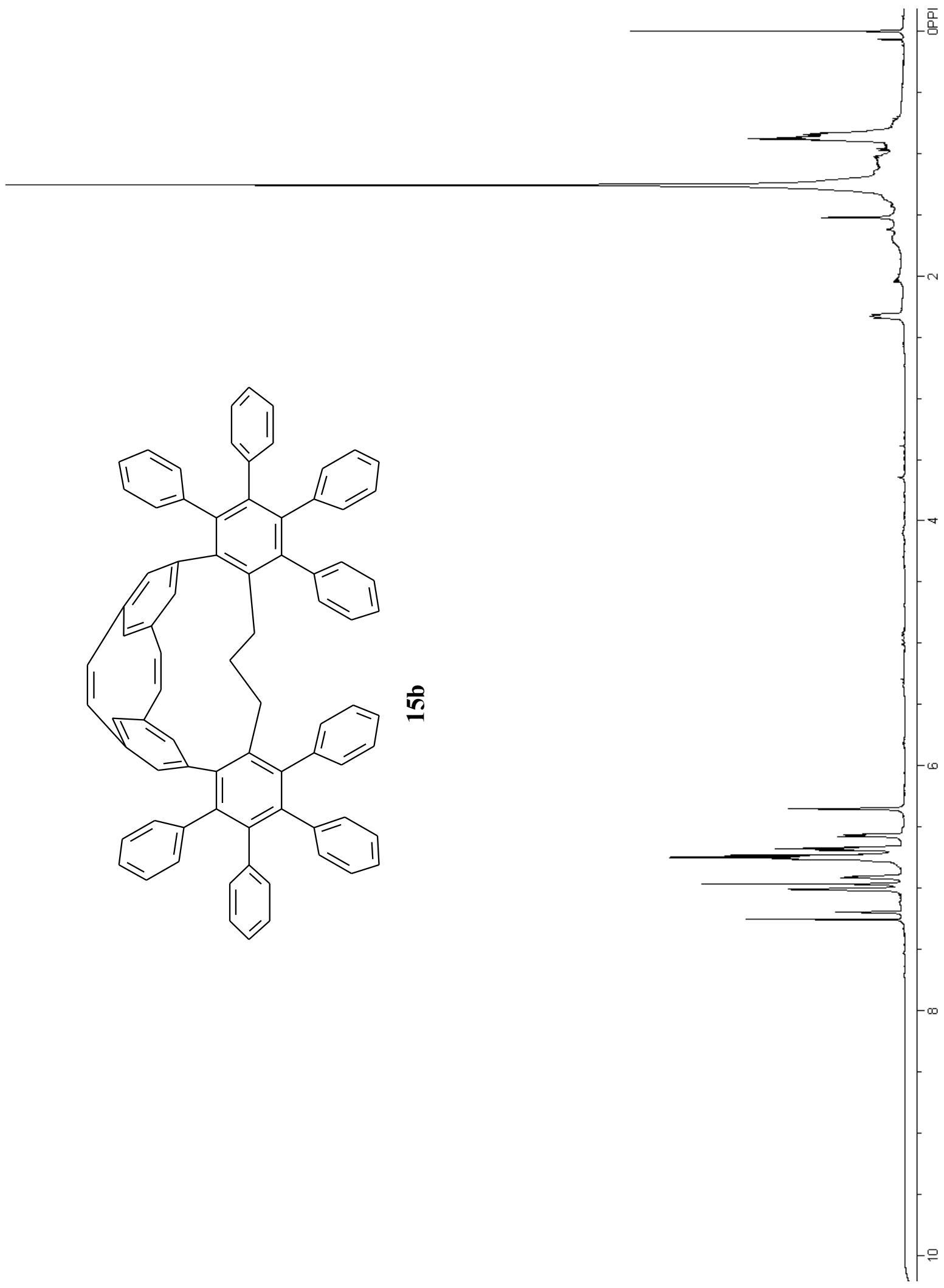




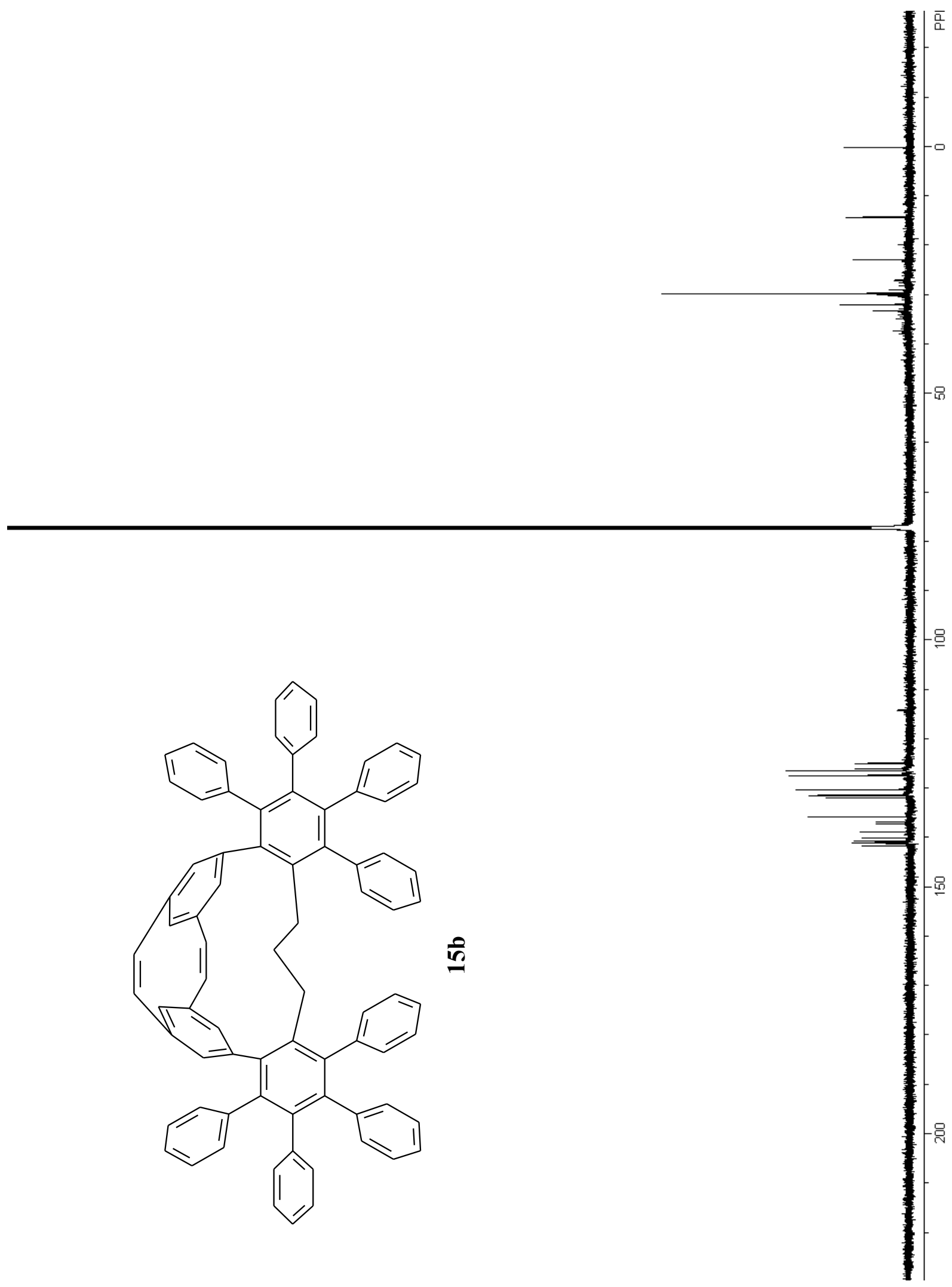




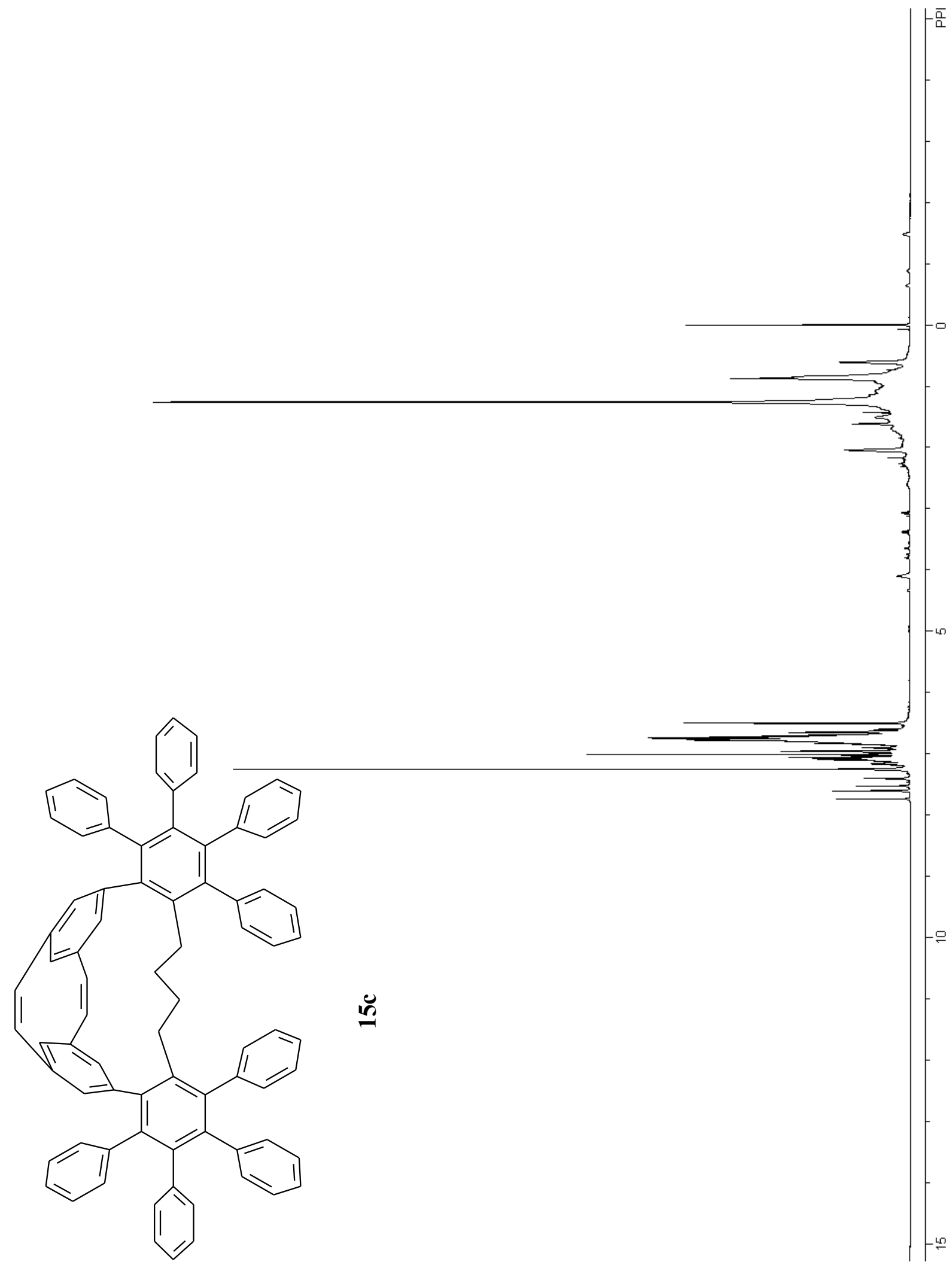




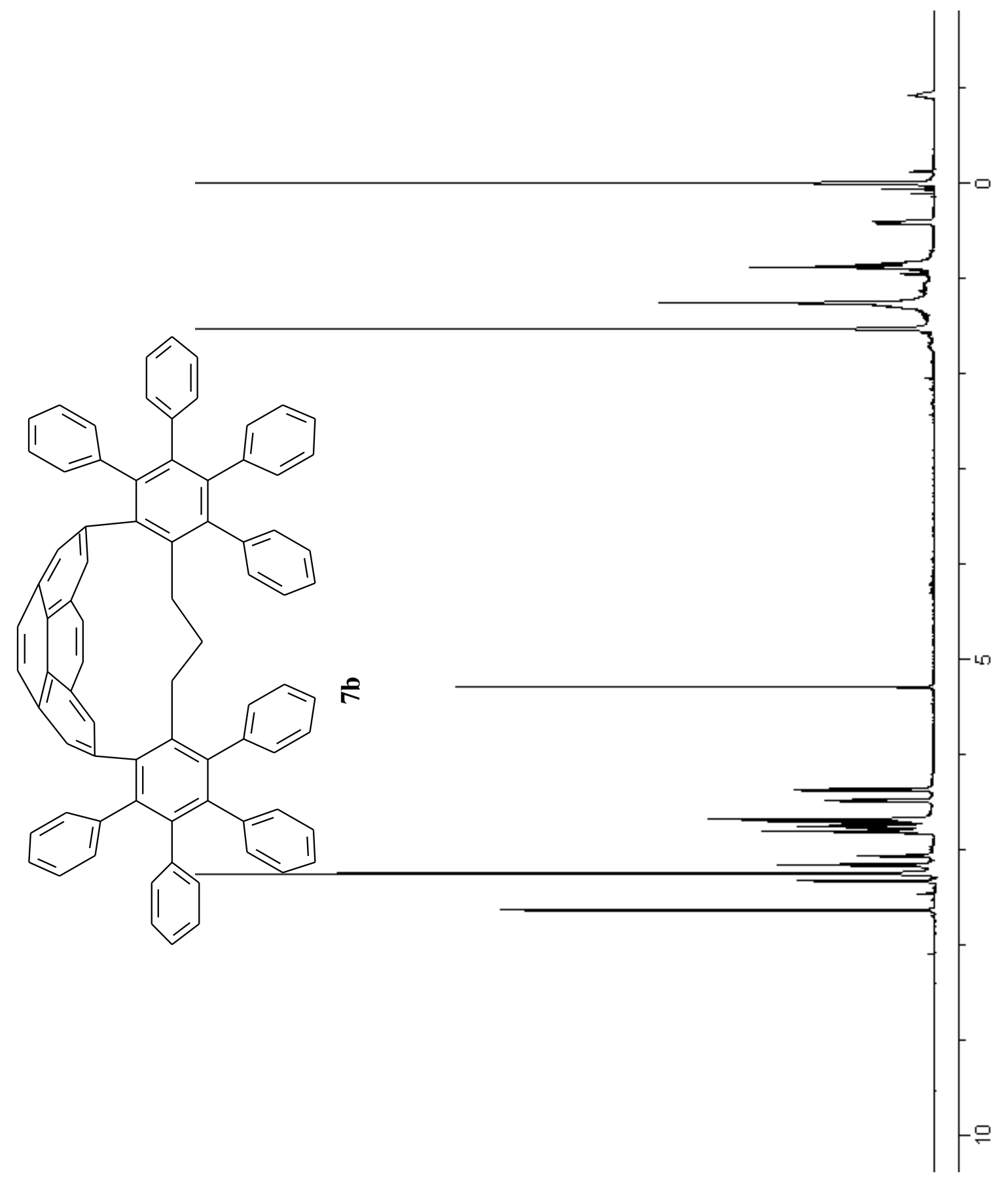




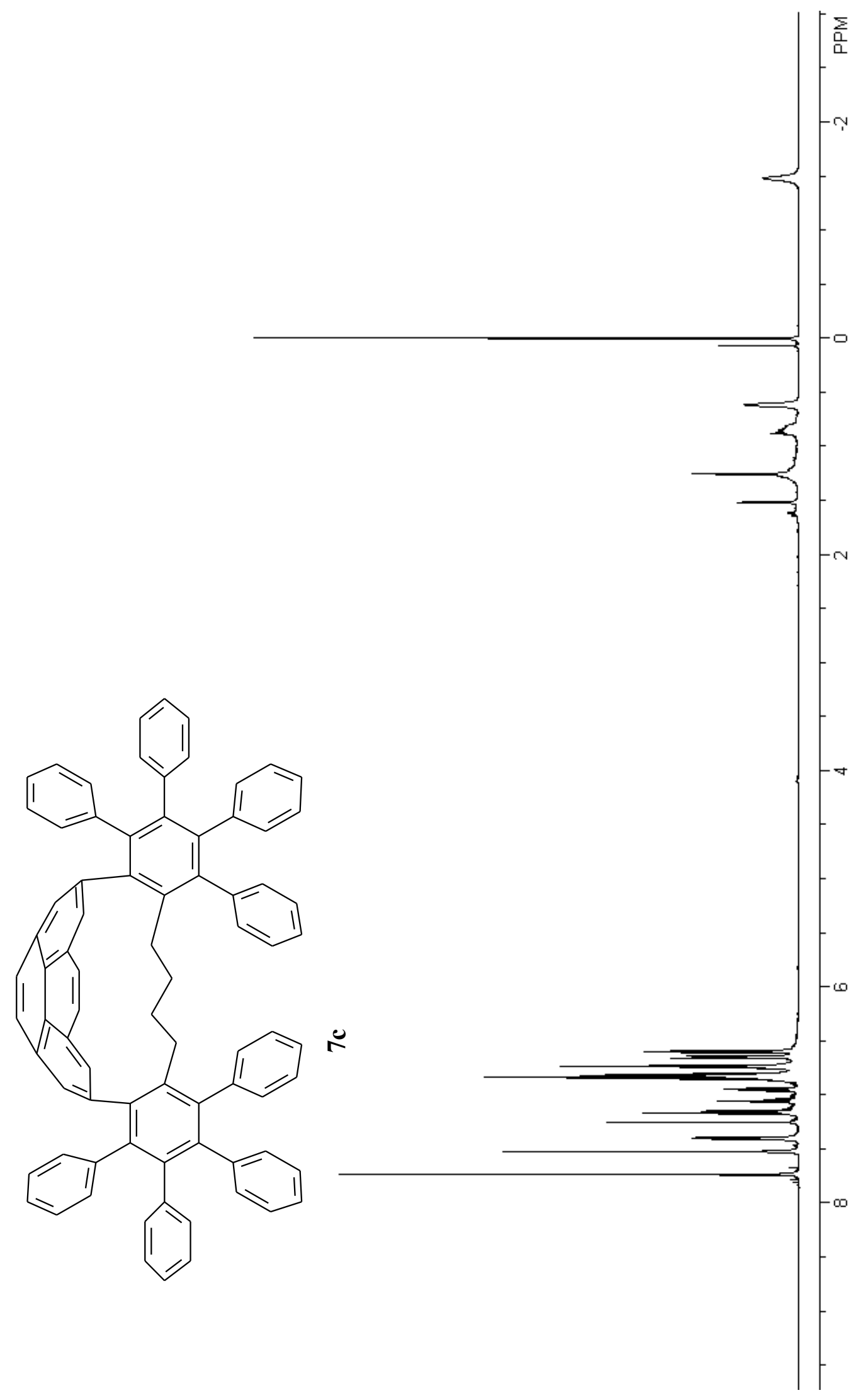




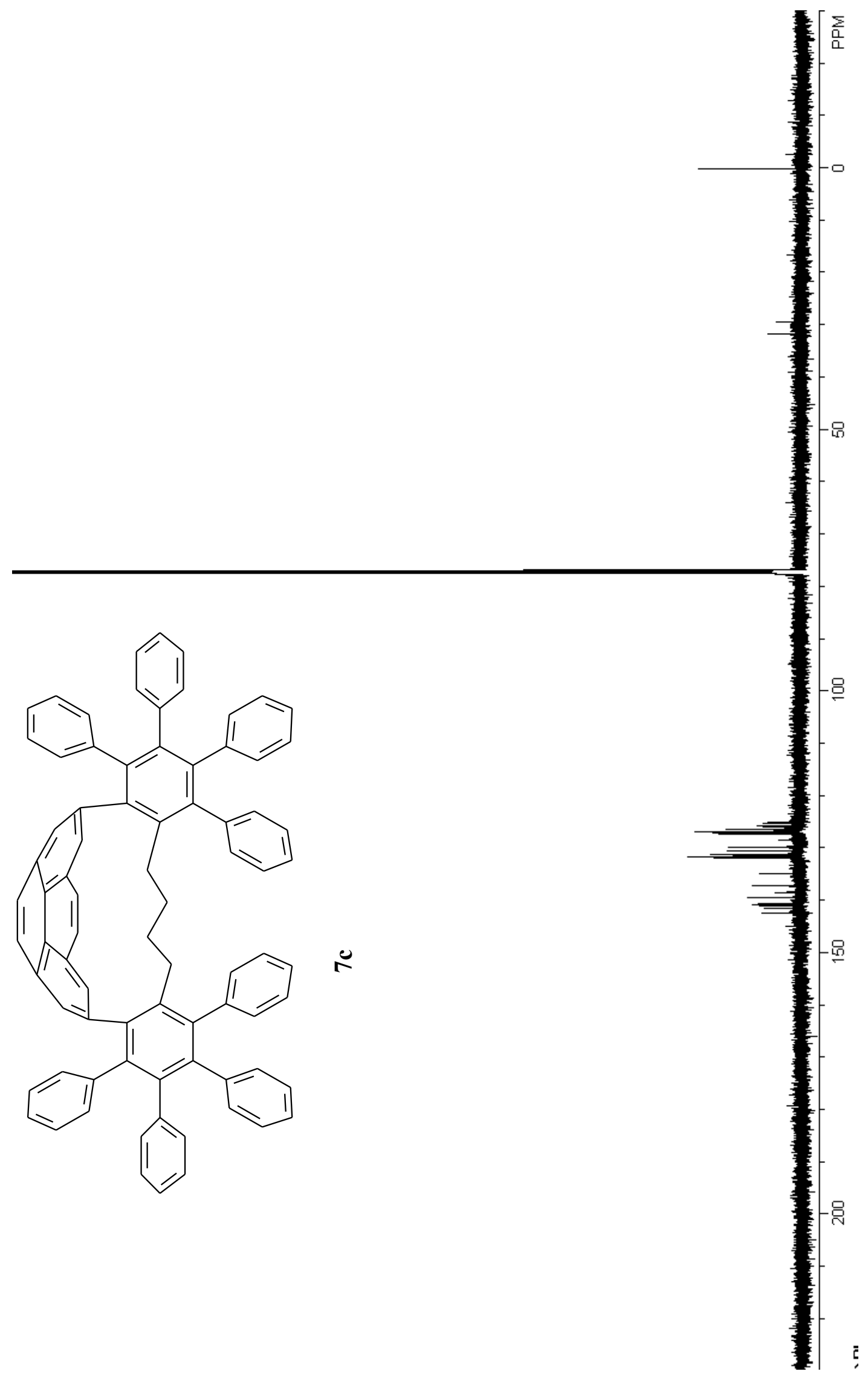




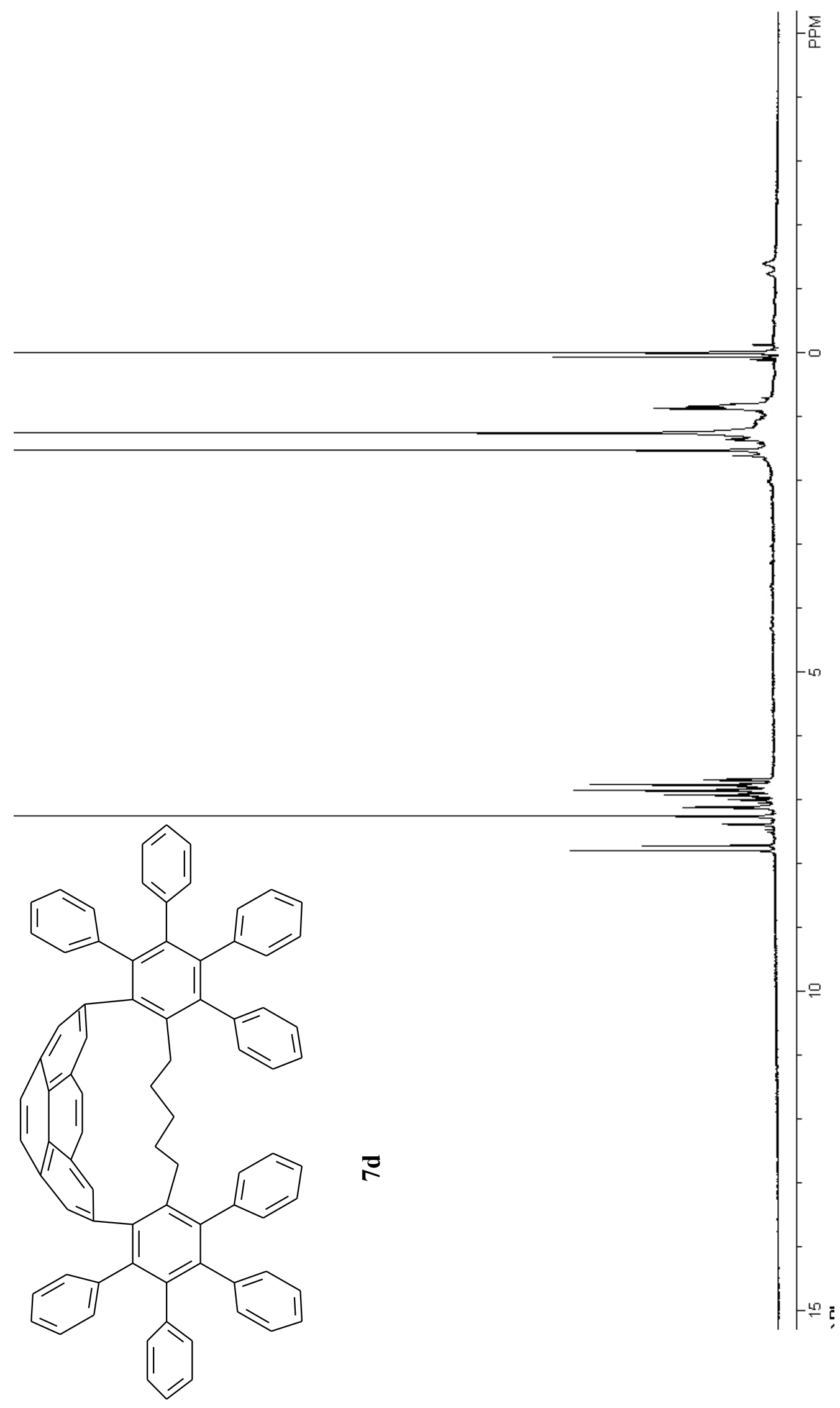



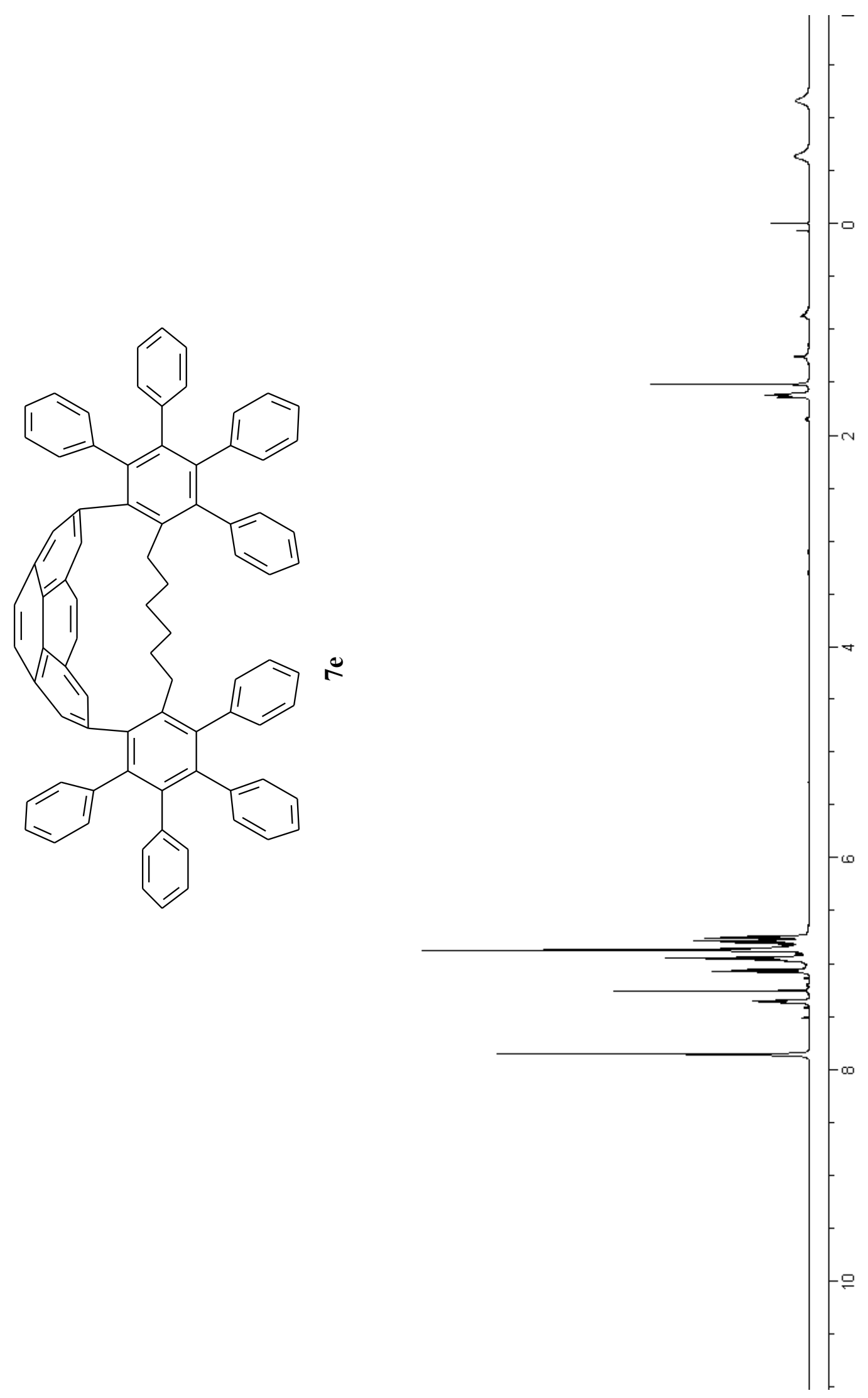


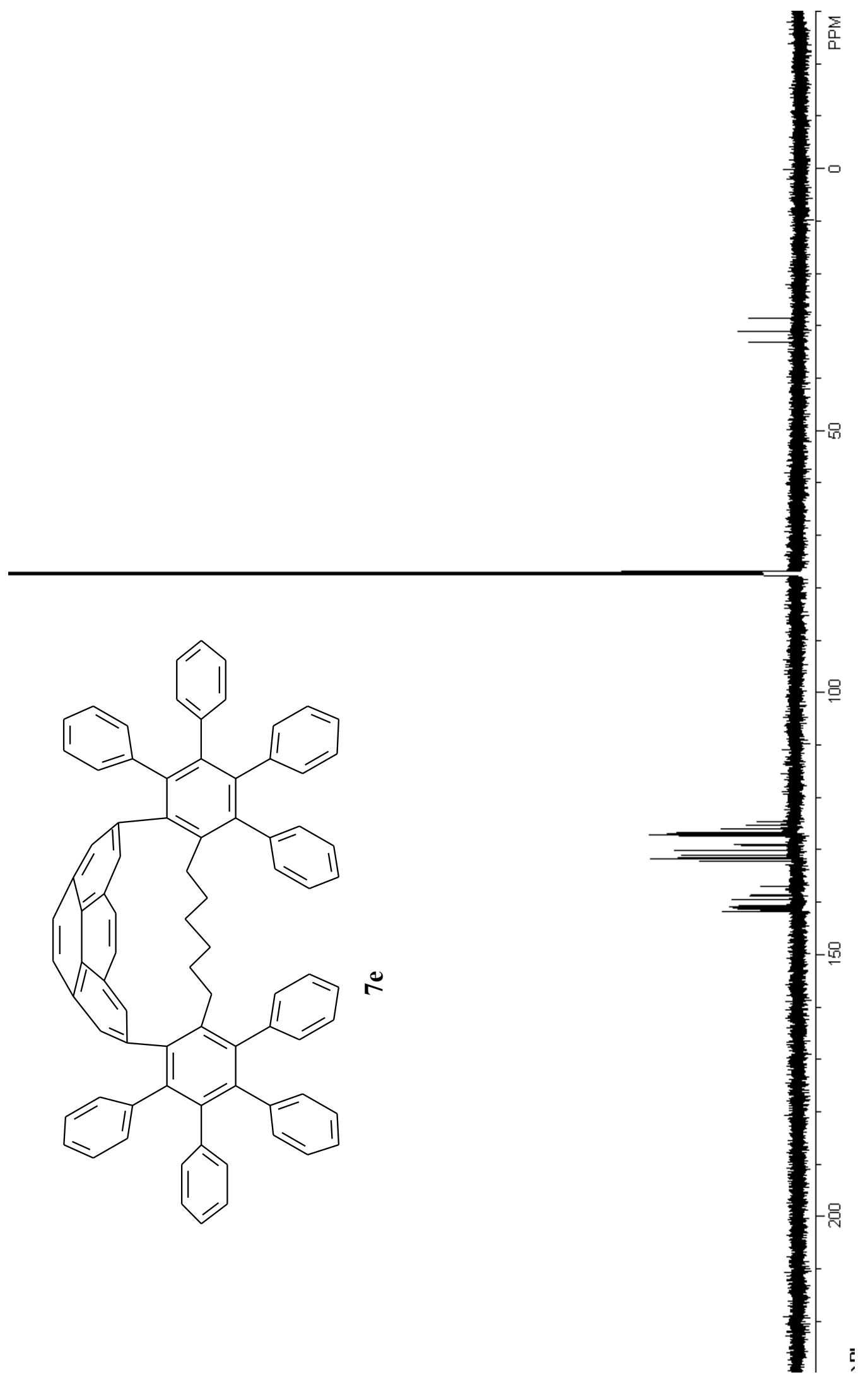


Table S1. Chemical shifts ( $\delta$ ppm) of the protons on 11a-e.

\begin{tabular}{|c|c|c|c|c|c|c|}
\hline Compound & $\mathrm{H}_{\mathrm{A}}$ & $\mathrm{H}_{\mathrm{B}}$ & $\mathrm{H}_{\mathrm{C}}$ & $\mathrm{H}_{\mathrm{D}}$ & $\mathrm{H}_{\mathrm{E}}$ & $\mathrm{H}_{\mathrm{F}}$ \\
\hline $11 a$ & 6.94 & 6.66 (br) & $4.43(\mathrm{de})$ & $\begin{array}{l}2.49(2.92)^{\mathrm{b}} \\
\Delta \delta=0.43\end{array}$ & - & - \\
\hline $11 \mathrm{~b}$ & 6.97 & $7.08^{\mathrm{a}}$ & $4.36(\mathrm{de})$ & $\begin{array}{l}2.05(2.62) \\
\Delta \delta=0.57\end{array}$ & $\begin{array}{l}1.21(1.96) \\
\Delta \delta=0.75\end{array}$ & - \\
\hline 11c & 7.00 & 6.87 & 4.43 & $\begin{array}{l}1.73(2.62) \\
\Delta \delta=0.89\end{array}$ & $\begin{array}{l}0.79(1.59) \\
\Delta \delta=0.80\end{array}$ & - \\
\hline 11d & $7.02 *$ & 6.90 & $4.35(\mathrm{de})$ & $\begin{array}{l}2.05(2.62) \\
\Delta \delta=0.57\end{array}$ & $\begin{array}{l}0.88(1.59) \\
\Delta \delta=0.71\end{array}$ & $\begin{array}{l}0.22(1.29) \\
\Delta \delta=1.07\end{array}$ \\
\hline $11 \mathrm{e}$ & $7.09 *$ & 6.96 & 4.43 & $\begin{array}{l}2.12(2.62) \\
\Delta \delta=0.50\end{array}$ & $\begin{array}{l}0.96(1.59) \\
\Delta \delta=0.63\end{array}$ & $\begin{array}{l}0.27(1.29) \\
\Delta \delta=1.02\end{array}$ \\
\hline
\end{tabular}

Notes: "br" denotes a broad signal; "de" denotes a virtually degenerate AB system; (a) denotes that the chemical shifts were determined by a low cut of the ${ }^{1} \mathrm{H},{ }^{1} \mathrm{H}-\mathrm{COSY}$ spectrum (for overlapping signals); (b) numbers in brackets are calculated values for the corresponding protons in the analogous $1, n$-diphenylalkanes.

Table S2. Chemical shifts ( $\delta$ ppm) of the protons on 12a-e.

\begin{tabular}{|c|c|c|c|c|c|c|}
\hline Compound & $\mathrm{H}_{\mathrm{A}}$ & $\mathrm{H}_{\mathrm{B}}$ & $\mathrm{H}_{\mathrm{C}}$ & $\mathrm{H}_{\mathrm{D}}$ & $\mathrm{H}_{\mathrm{E}}$ & $\mathrm{H}_{\mathrm{F}}$ \\
\hline $12 a$ & - & - & $\begin{array}{l}4.22,4.16 \\
\text { (AB) }\end{array}$ & $\begin{array}{l}2.46(2.92)^{\mathrm{a}} \\
\Delta \delta=0.46\end{array}$ & - & - \\
\hline $12 \mathrm{~b}$ & 7.15 & 7.06 & $4.39(\mathrm{~s})$ & $\begin{array}{l}1.86(2.62) \\
\Delta \delta=0.76\end{array}$ & $\begin{array}{l}1.21(1.96) \\
\Delta \delta=0.75\end{array}$ & - \\
\hline $12 \mathrm{c}$ & 7.01 & 6.96 & $4.26(\mathrm{~s})$ & $\begin{array}{l}1.70(2.62) \\
\Delta \delta=0.92\end{array}$ & $\begin{array}{l}0.81(1.59) \\
\Delta \delta=0.78\end{array}$ & - \\
\hline 12d & - & - & $\begin{array}{l}4.26,4.25 \\
(\mathrm{AB})\end{array}$ & $\begin{array}{l}2.05(2.62) \\
\Delta \delta=0.57\end{array}$ & $\begin{array}{l}0.88(1.59) \\
\Delta \delta=0.72\end{array}$ & $\begin{array}{l}0.28(1.29) \\
\Delta \delta=1.01\end{array}$ \\
\hline $12 \mathrm{e}$ & - & - & $\begin{array}{l}4.28,4.26 \\
\text { (AB) }\end{array}$ & $\begin{array}{l}2.10(2.62) \\
\Delta \delta=0.52\end{array}$ & $\begin{array}{l}0.97(1.59) \\
\Delta \delta=0.62\end{array}$ & $\begin{array}{l}0.33(1.29) \\
\Delta \delta=0.96\end{array}$ \\
\hline
\end{tabular}

Note: "AB" denotes an $\mathrm{AB}$ system; (a) numbers in brackets are calculated values for the corresponding protons in the analogous $1, n$-diphenylalkanes. 


\section{Calculated Structures of 2a-e, 3a-e and 13a-e}
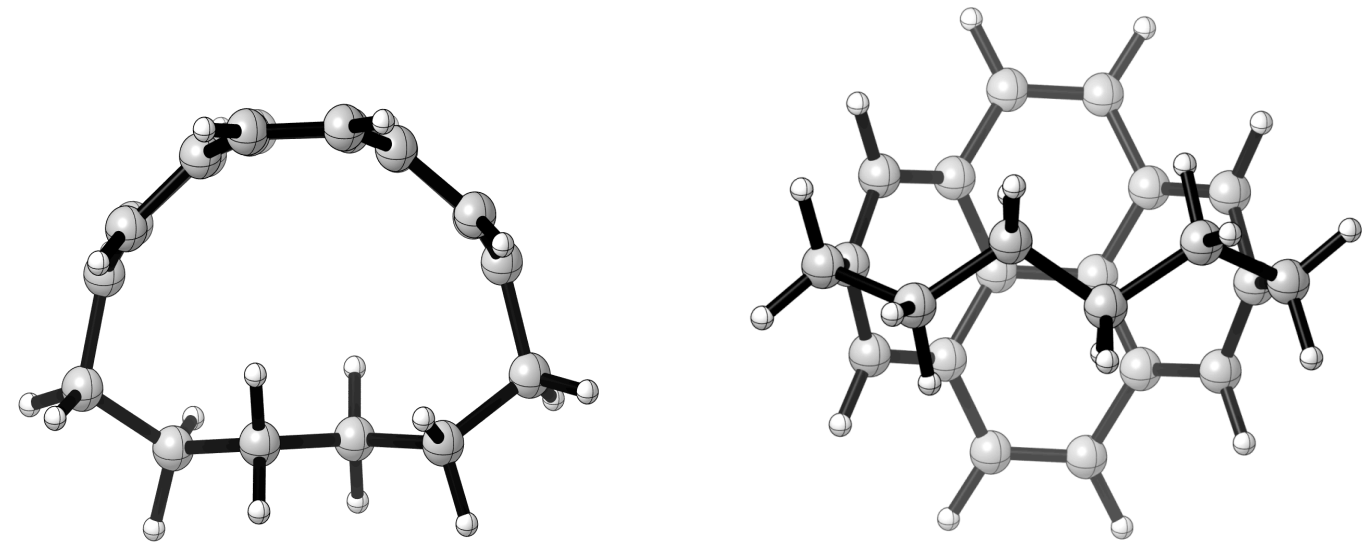

Figure S1. Calculated Structure of $\mathbf{2 a}$.
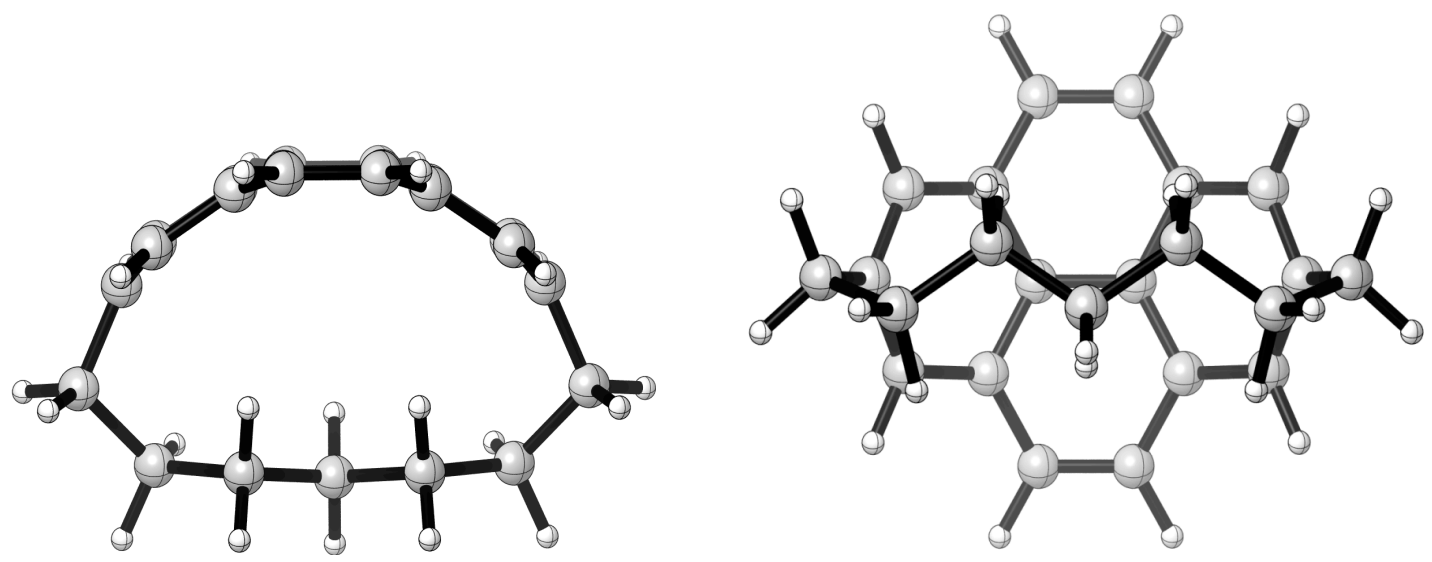

Figure S2. Calculated Structure of $\mathbf{2 b}$.
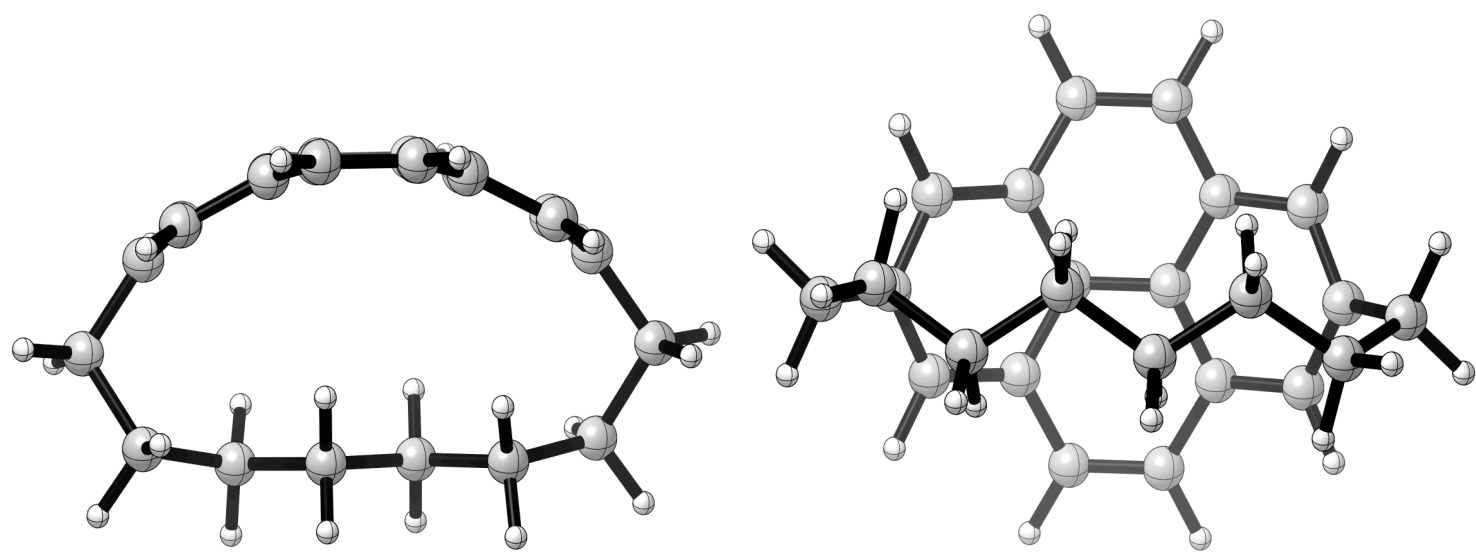

Figure S3. Calculated Structure of 2c. 

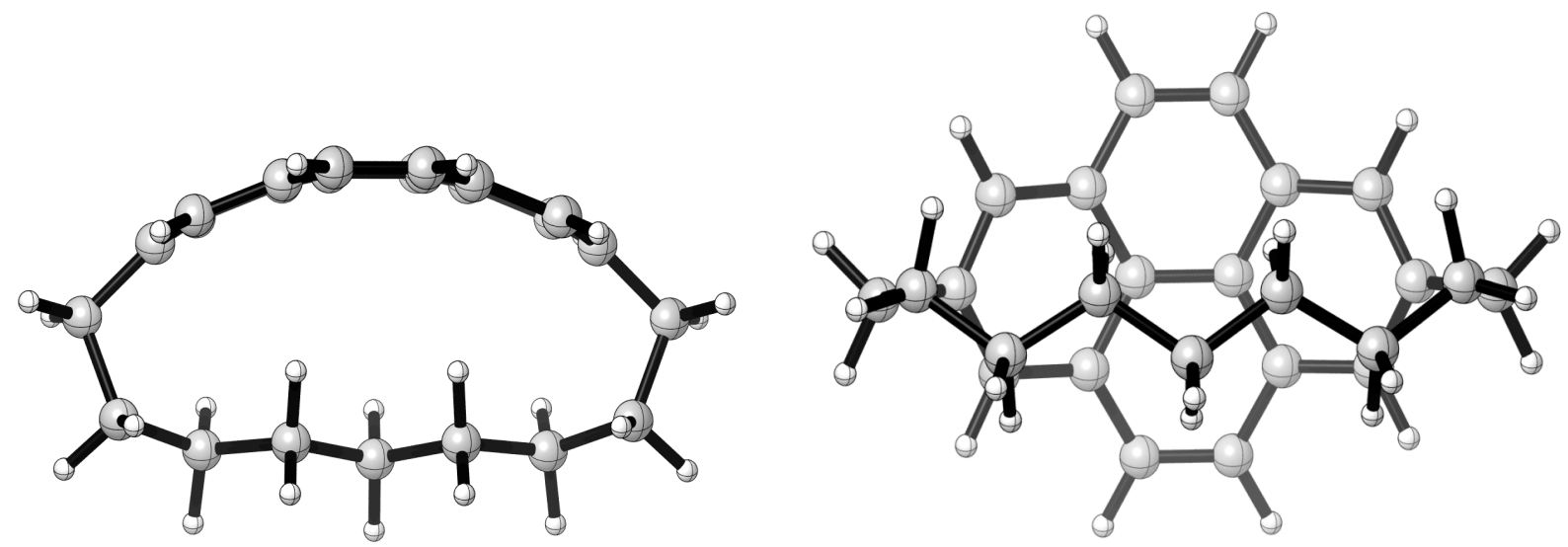

Figure S4. Calculated Structure of 2d.
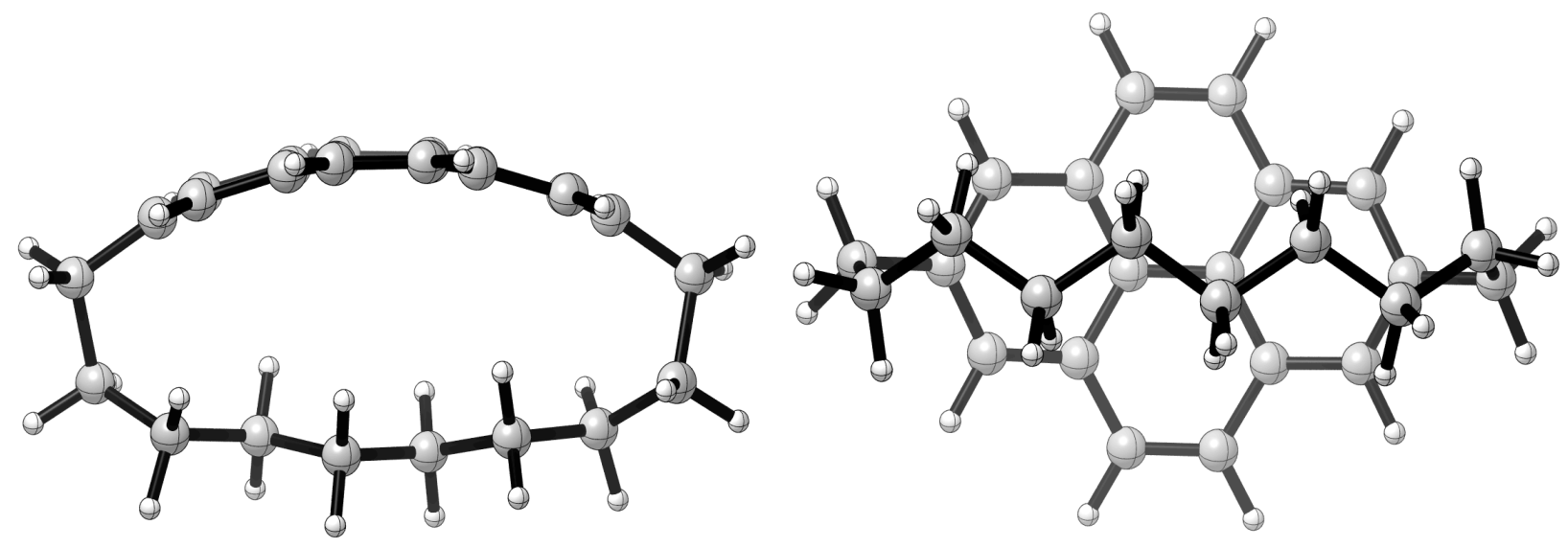

Figure S5. Calculated Structure of 2 e.
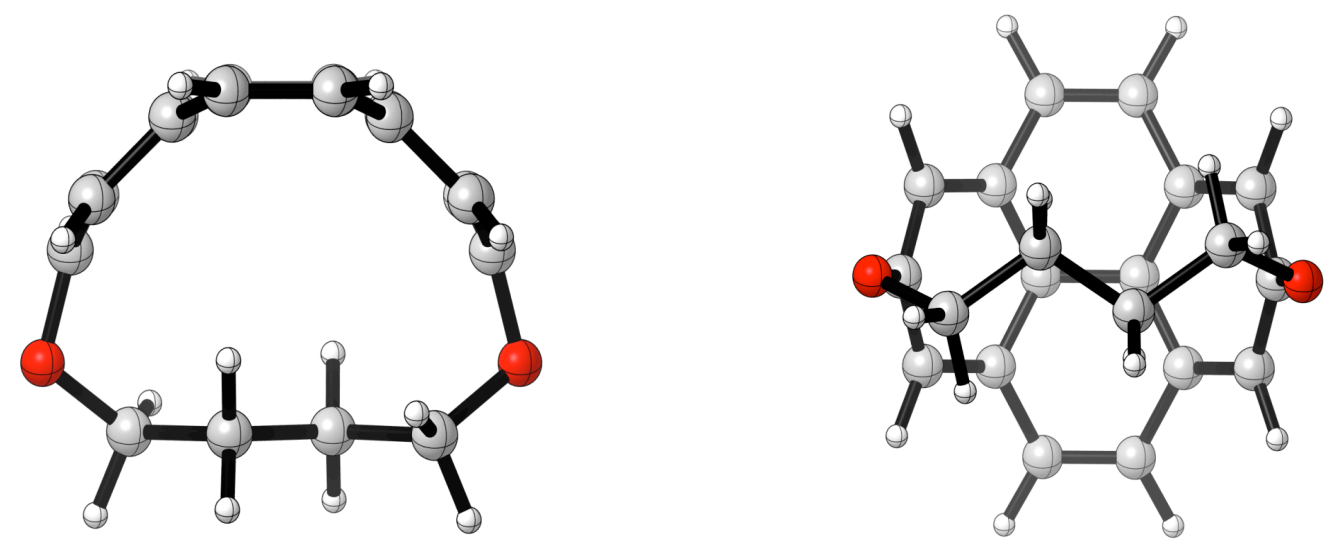

Figure S6. Calculated Structure of 3a. 

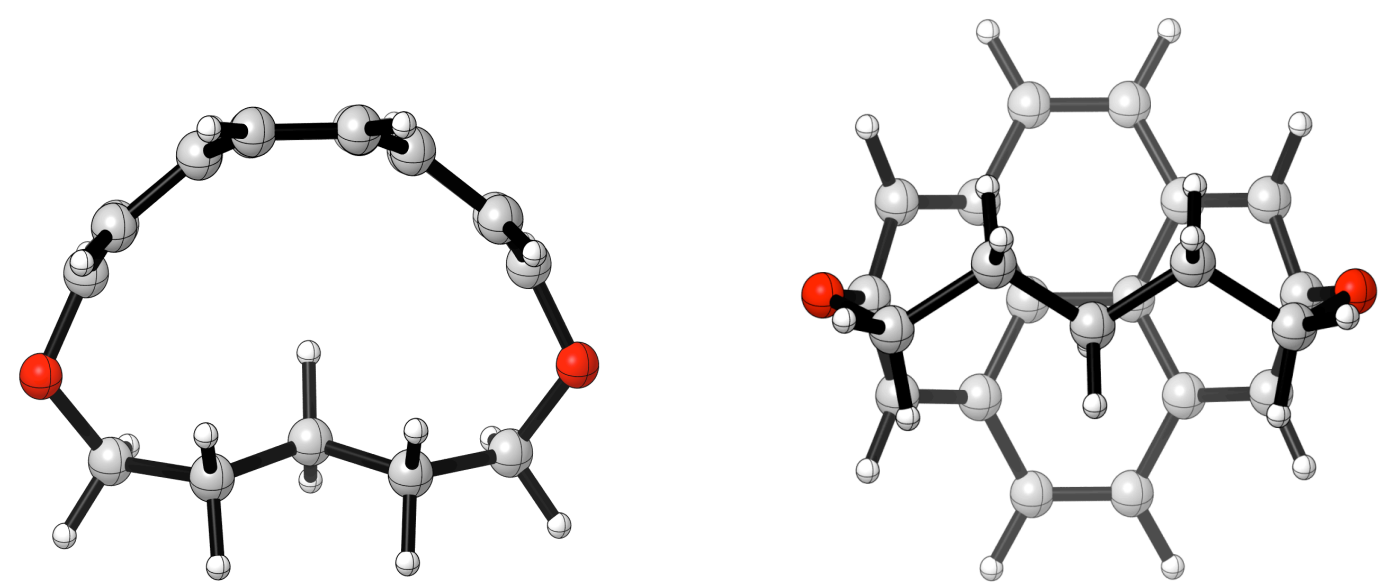

Figure S7. Calculated Structure of $\mathbf{3 b}$.
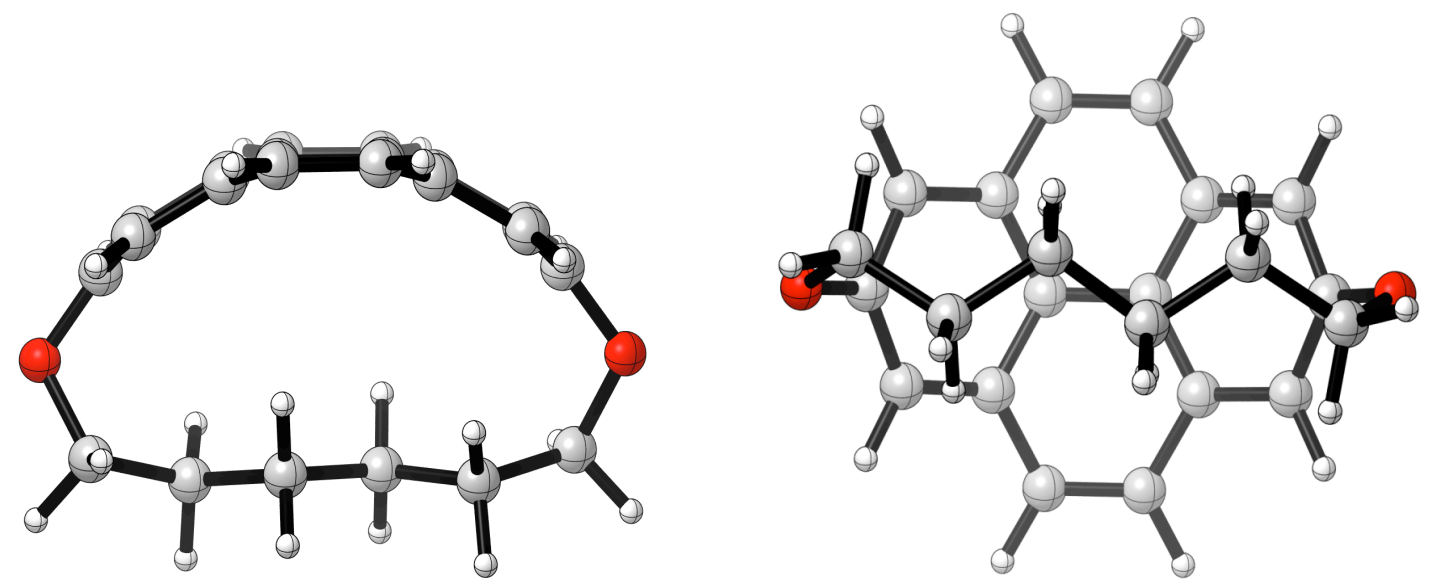

Figure S8. Calculated Structure of $\mathbf{3 c}$.
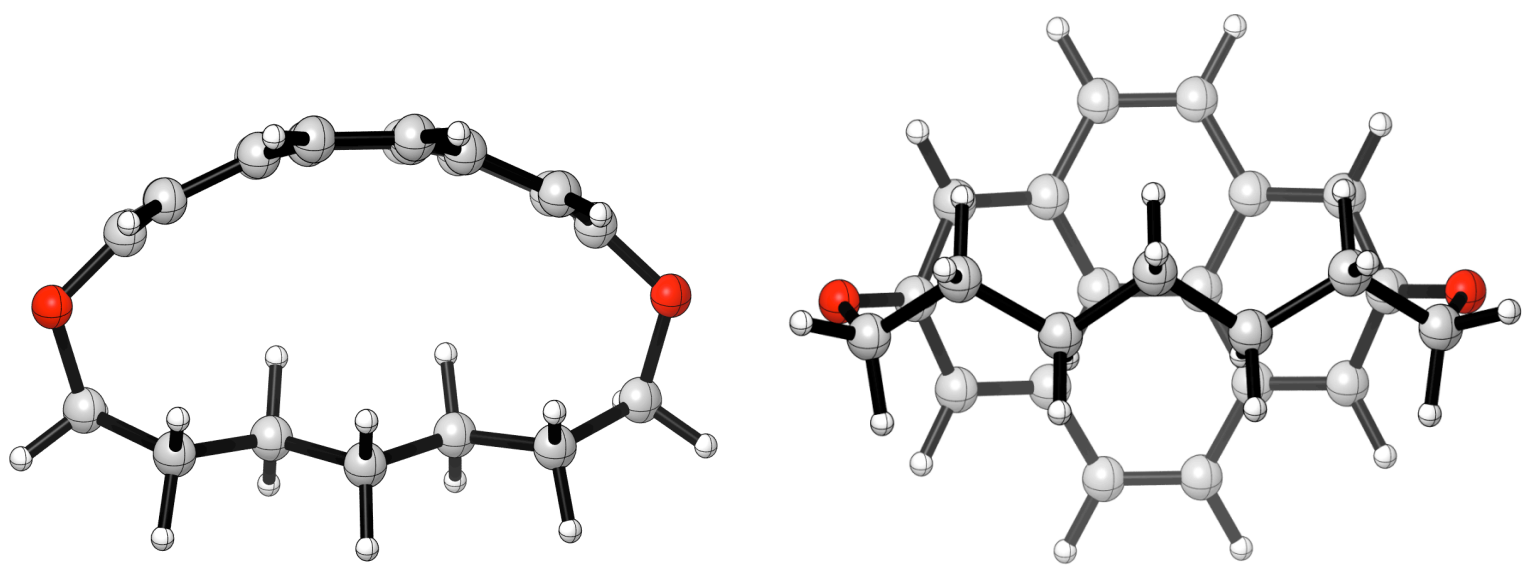

Figure S9. Calculated Structure of 3d. 

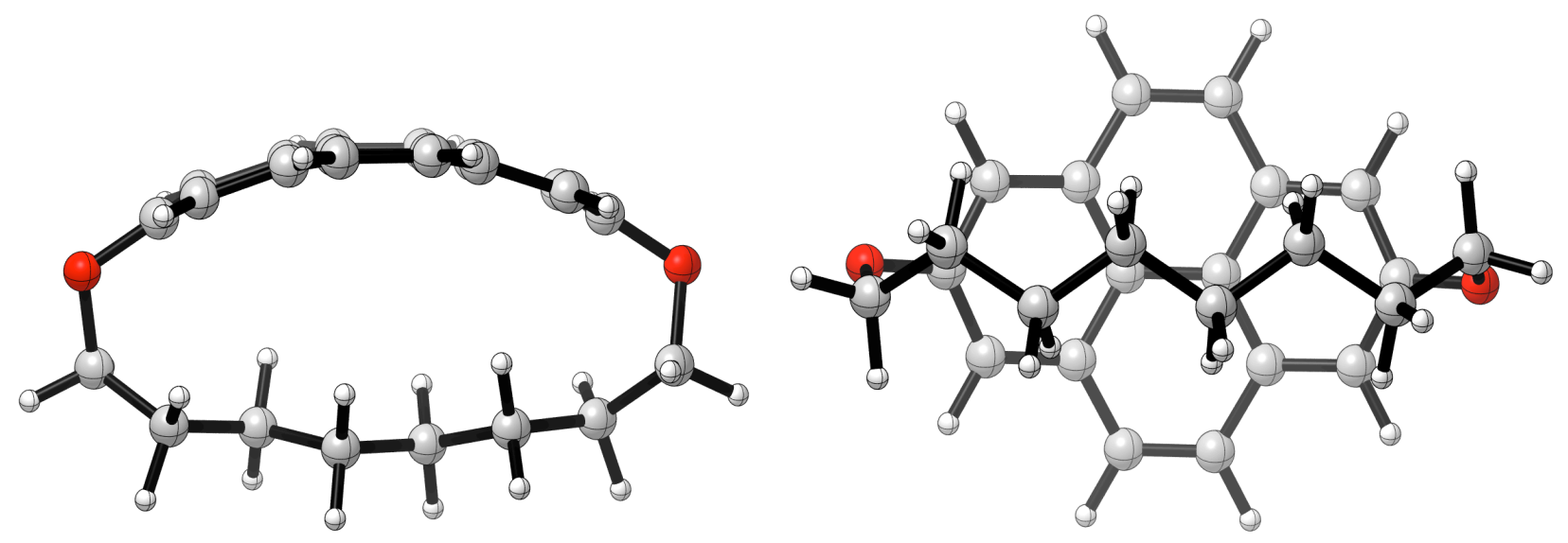

Figure S10. Calculated Structure of 3e.

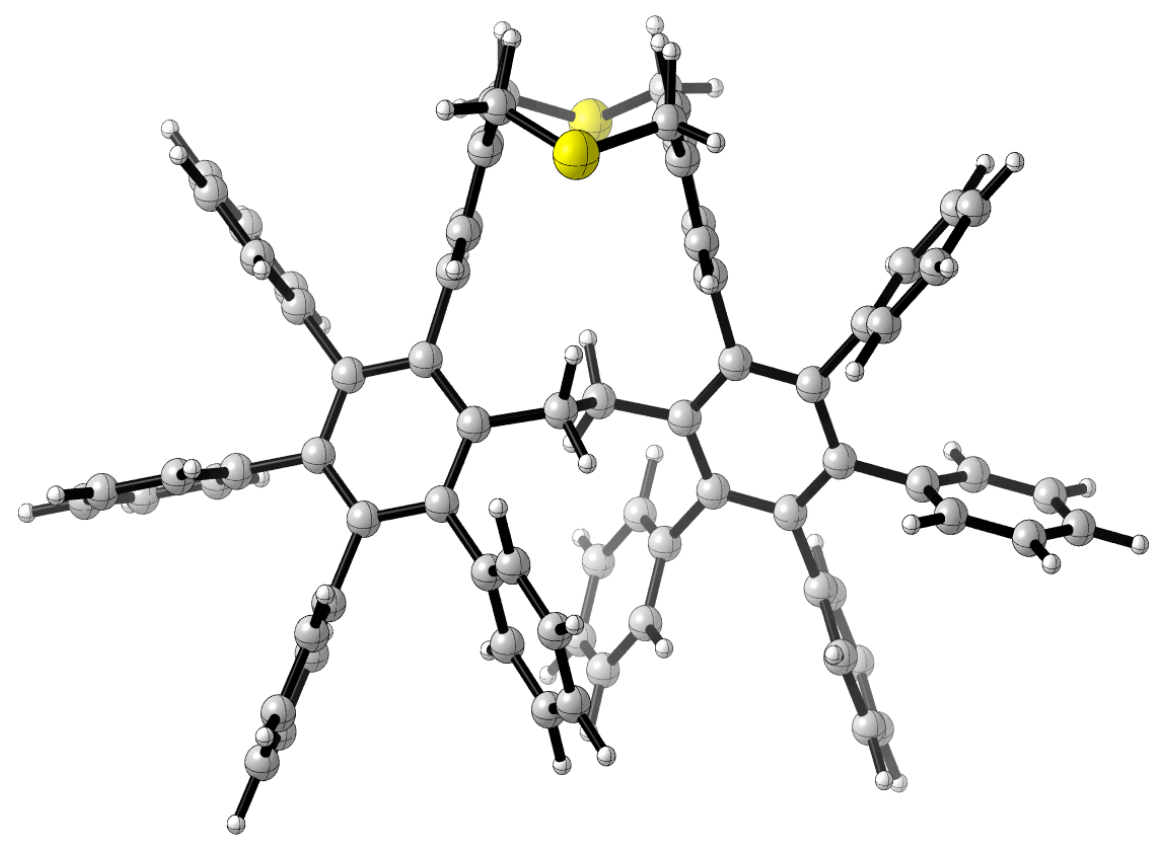

Figure S11. Calculated Structure of 13a. 


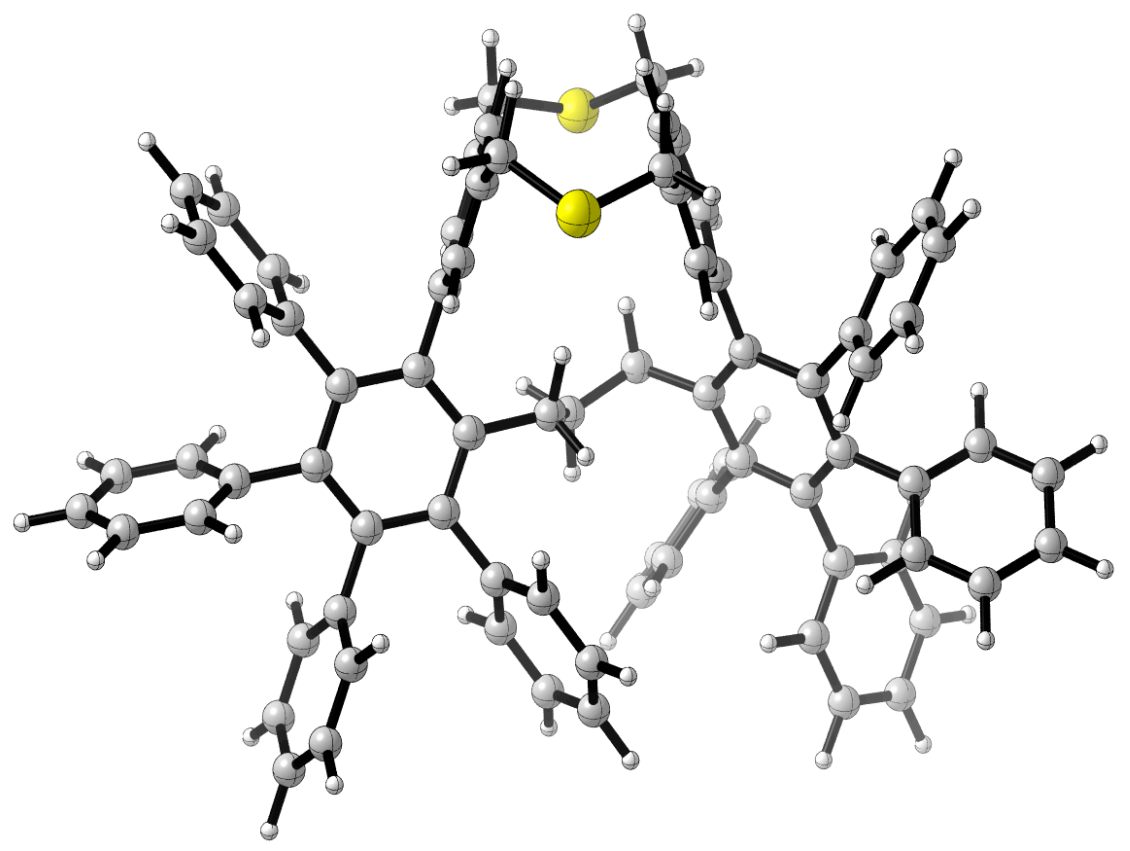

Figure S12. Calculated Structure of 13b.

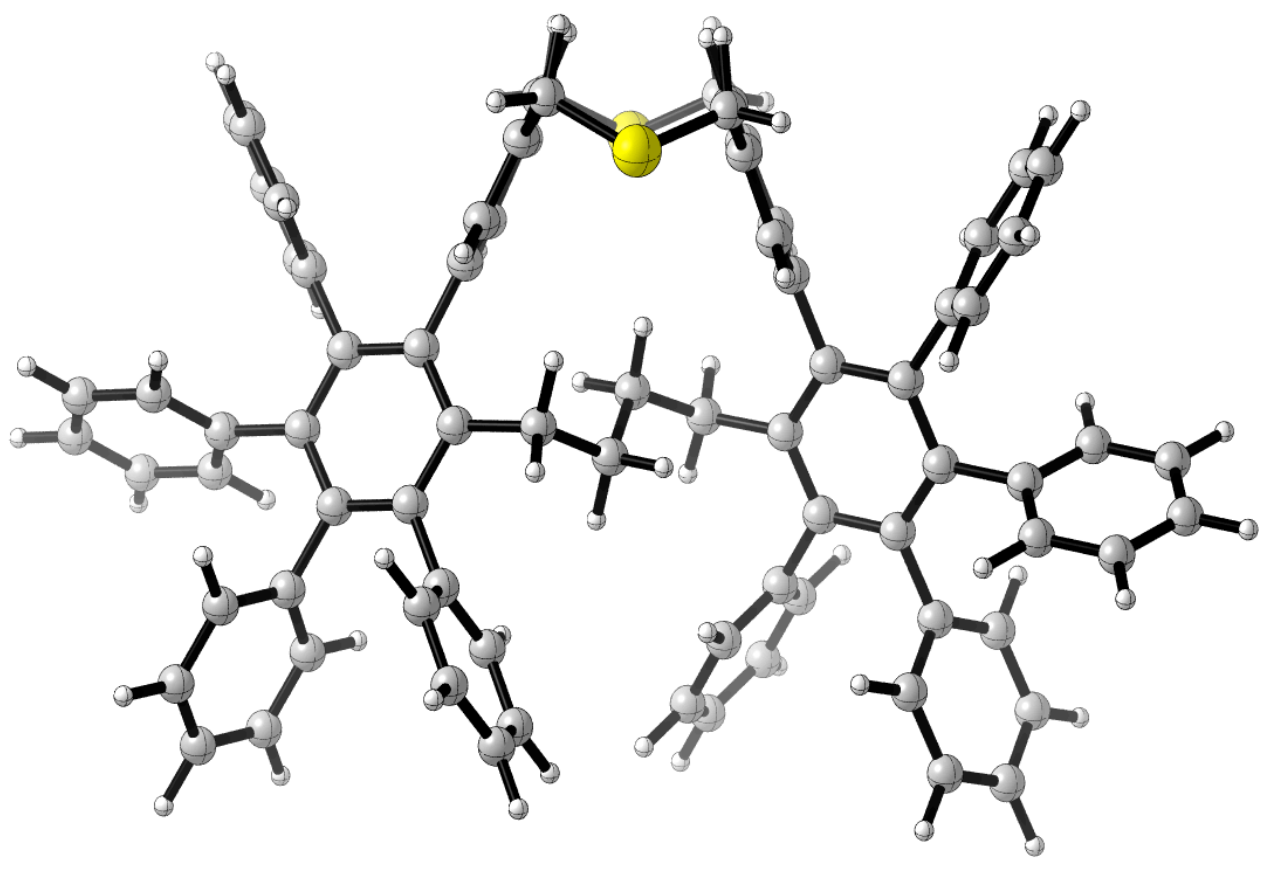

Figure S13. Calculated Structure of 13c. 


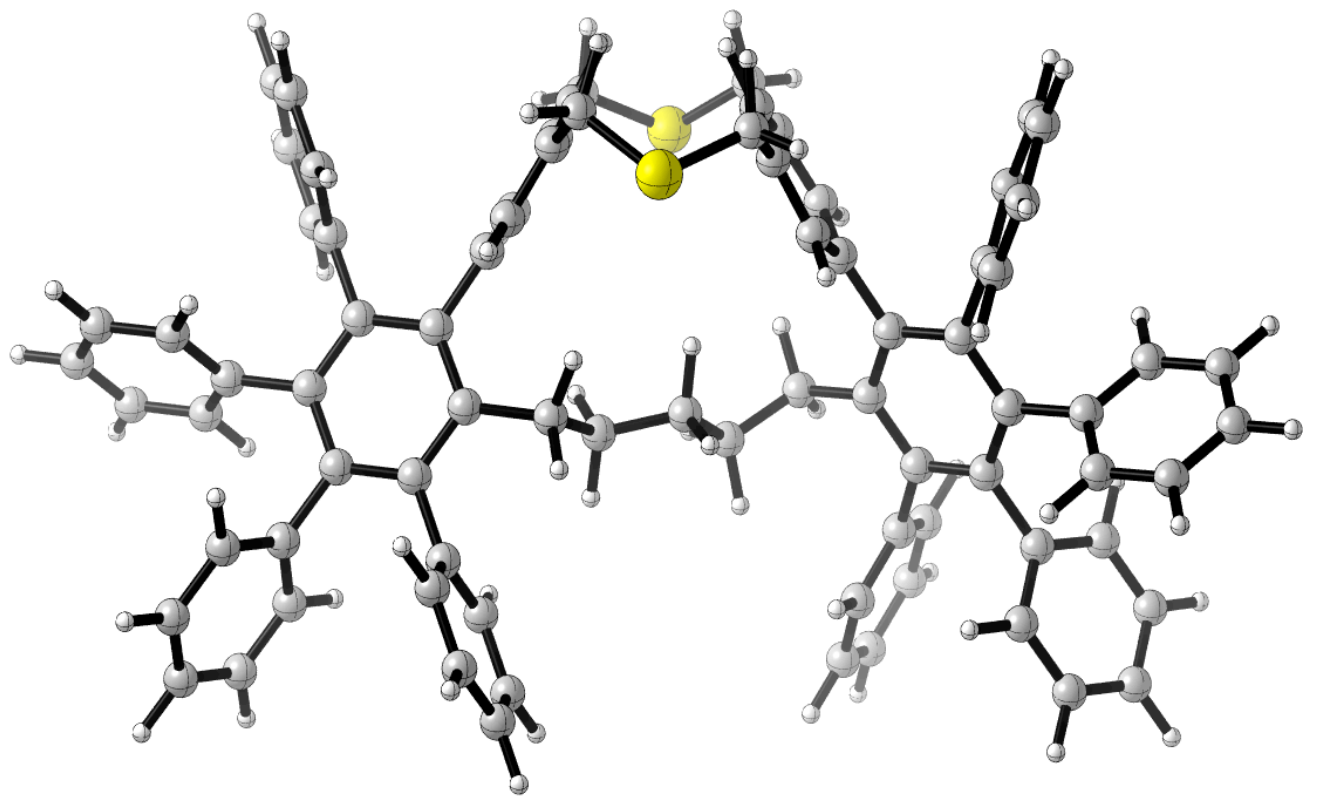

Figure S14. Calculated Structure of 13d.

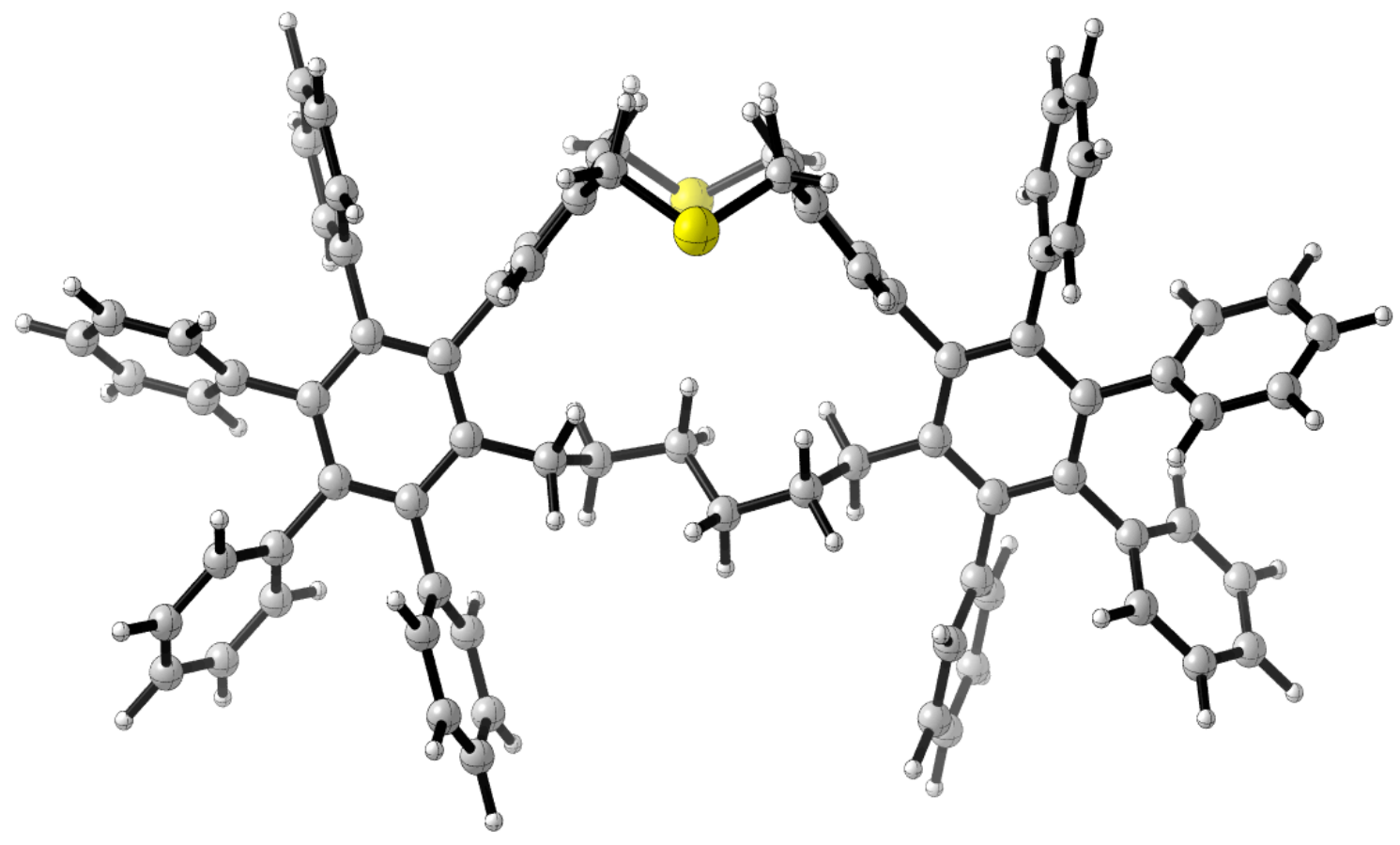

Figure S15. Calculated Structure of 13e. 


\section{Computational Details and Cartesian Co-ordinates for Calculated Structures of 2a-e, 3a- e, 13a-e and 7a-e}

\section{DFT Calculations of Pyrenophanes 7a-e}

The molecular structures of pyrenophanes 2a-e, 3a-e, 4, 7a-e, and compounds 13a-e were first constructed and pre-optimized at the AM1 level using Spartan'10 software package (Wavefun, Inc.). The structures were then optimized by DFT calculations at the B3LYP/6$31 \mathrm{G}(\mathrm{d})$ level of theory using Gaussian'09 software package. Visualization of molecular structures and frontier molecular orbitals was done by CYLview and GaussView 5.0 software packages. The optimized Cartesian coordinates and total energies of the compounds are provided below.

Compound 2a: $\quad E(R B 3 L Y P)=-850.33990530$ a.u.

$\begin{array}{lrrr}\text { C } & -0.42765300 & 0.57309100 & -1.60678100 \\ \mathrm{C} & -1.71358600 & 2.27415800 & 0.16156200 \\ \mathrm{C} & 0.15703300 & 1.84380100 & -1.33396700 \\ \mathrm{C} & -1.81715500 & 0.37565600 & -1.35137300 \\ \mathrm{C} & -2.45167800 & 1.26240600 & -0.47238600 \\ \mathrm{C} & -0.51256500 & 2.68497800 & -0.43099100 \\ \mathrm{H} & -3.43589400 & 0.99632100 & -0.09011700 \\ \mathrm{H} & 0.02820300 & 3.53298500 & -0.01456000 \\ \mathrm{C} & 0.42765300 & -0.57309100 & -1.60678100 \\ \mathrm{C} & 1.71358600 & -2.27415800 & 0.16156200 \\ \mathrm{C} & 1.81715500 & -0.37565600 & -1.35137300 \\ \mathrm{C} & -0.15703300 & -1.84380100 & -1.33396700 \\ \mathrm{C} & 0.51256500 & -2.68497800 & -0.43099100 \\ \mathrm{C} & 2.45167800 & -1.26240600 & -0.47238600 \\ \mathrm{H} & -0.02820300 & -3.53298500 & -0.01456000 \\ \mathrm{H} & 3.43589400 & -0.99632100 & -0.09011700 \\ \mathrm{C} & -1.56691300 & -2.01765800 & -1.61746100 \\ \mathrm{H} & -1.97947100 & -3.02285600 & -1.66010000 \\ \mathrm{C} & -2.38062600 & -0.92925600 & -1.63320200 \\ \mathrm{H} & -3.46084500 & -1.04051400 & -1.68901300 \\ \mathrm{C} & 2.38062600 & 0.92925600 & -1.63320200 \\ \mathrm{H} & 3.46084500 & 1.04051400 & -1.68901300 \\ \mathrm{C} & 1.56691300 & 2.01765800 & -1.61746100 \\ \mathrm{H} & 1.97947100 & 3.02285600 & -1.66010000 \\ \mathrm{C} & 1.95956400 & -2.53885500 & 1.63417600 \\ \mathrm{C} & -1.95956400 & 2.53885500 & 1.63417600 \\ \mathrm{C} & 1.56691300 & -1.25609400 & 2.46062600 \\ \mathrm{H} & 2.21547400 & -0.44445500 & 2.10770200 \\ \mathrm{H} & 1.84992000 & -1.43364900 & 3.50708700 \\ \mathrm{C} & 0.08531200 & -0.77329100 & 2.40205800 \\ \mathrm{H} & -0.39754100 & -1.15762200 & 1.49680900 \\ \mathrm{H} & -0.46924500 & -1.20536100 & 3.24692800\end{array}$




$\begin{array}{lrrr}\mathrm{C} & -0.08531200 & 0.77329100 & 2.40205800 \\ \mathrm{H} & 0.39754100 & 1.15762200 & 1.49680900 \\ \mathrm{H} & 0.46924500 & 1.20536100 & 3.24692800 \\ \mathrm{C} & -1.56691300 & 1.25609400 & 2.46062600 \\ \mathrm{H} & -1.84992000 & 1.43364900 & 3.50708700 \\ \mathrm{H} & -2.21547400 & 0.44445500 & 2.10770200 \\ \mathrm{H} & -1.37672700 & 3.40367400 & 1.97553200 \\ \mathrm{H} & -3.01537000 & 2.75473900 & 1.84616500 \\ \mathrm{H} & 1.37672700 & -3.40367400 & 1.97553200 \\ \mathrm{H} & 3.01537000 & -2.75473900 & 1.84616500\end{array}$

Compound 2b: $\quad \mathrm{E}(\mathrm{RB} 3 \mathrm{LYP})=-889.68153441$ a.u.

$\begin{array}{lrrr}\mathrm{C} & 0.11710700 & -0.01882300 & 3.05894400 \\ \mathrm{H} & 0.15376700 & 2.13938700 & 3.01165700 \\ \mathrm{C} & 0.56518300 & 1.22489600 & 2.58739600 \\ \mathrm{C} & 1.50001800 & -1.14519600 & 1.40007000 \\ \mathrm{C} & 1.30238100 & 1.31019400 & 1.40021600 \\ \mathrm{C} & 0.74527600 & -1.17540700 & 2.58422800 \\ \mathrm{C} & 1.61190500 & 0.09803400 & 0.71312500 \\ \mathrm{C} & 1.44183200 & 2.55614900 & 0.67985300 \\ \mathrm{H} & 0.46868300 & -2.14288400 & 2.99951000 \\ \mathrm{C} & 1.44183200 & 2.55614900 & -0.67985300 \\ \mathrm{H} & 1.40688300 & 3.49060800 & 1.23486300 \\ \mathrm{H} & 1.40688300 & 3.49060800 & -1.23486300 \\ \mathrm{C} & 1.30238100 & 1.31019400 & -1.40021600 \\ \mathrm{C} & 0.56518300 & 1.22489600 & -2.58739600 \\ \mathrm{C} & 1.61190500 & 0.09803400 & -0.71312500 \\ \mathrm{C} & 0.11710700 & -0.01882300 & -3.05894400 \\ \mathrm{H} & 0.15376700 & 2.13938700 & -3.01165700 \\ \mathrm{C} & 0.74527600 & -1.17540700 & -2.58422800 \\ \mathrm{H} & 0.46868300 & -2.14288400 & -2.99951000 \\ \mathrm{C} & 1.50001800 & -1.14519600 & -1.40007000 \\ \mathrm{C} & 1.82683800 & -2.35346200 & 0.68058300 \\ \mathrm{H} & 1.93145200 & -3.28336400 & 1.23457100 \\ \mathrm{C} & 1.82683800 & -2.35346200 & -0.68058300 \\ \mathrm{H} & 1.93145200 & -3.28336400 & -1.23457100 \\ \mathrm{C} & -1.25574700 & -0.11381700 & -3.69210300 \\ \mathrm{C} & -1.25574700 & -0.11381700 & 3.69210300 \\ \mathrm{C} & -2.34131900 & 0.23193500 & -2.61005600 \\ \mathrm{H} & -3.32646100 & 0.17467400 & -3.09155100 \\ \mathrm{H} & -2.19770400 & 1.28681200 & -2.34392100 \\ \mathrm{C} & -2.33995900 & -0.62045400 & -1.30279500 \\ \mathrm{H} & -1.39851300 & -1.17436000 & -1.22563800 \\ \mathrm{H} & -3.13059900 & -1.38115000 & -1.36097700\end{array}$




$\begin{array}{lrrr}\mathrm{C} & -2.34131900 & 0.23193500 & 2.61005600 \\ \mathrm{H} & -2.19770400 & 1.28681200 & 2.34392100 \\ \mathrm{H} & -3.32646100 & 0.17467400 & 3.09155100 \\ \mathrm{C} & -2.33995900 & -0.62045400 & 1.30279500 \\ \mathrm{H} & -3.13059900 & -1.38115000 & 1.36097700 \\ \mathrm{H} & -1.39851300 & -1.17436000 & 1.22563800 \\ \mathrm{C} & -2.50821500 & 0.20221300 & 0.00000000 \\ \mathrm{H} & -3.48583000 & 0.70739100 & 0.00000000 \\ \mathrm{H} & -1.75230100 & 0.99960900 & 0.00000000 \\ \mathrm{H} & -1.42525700 & -1.12264000 & 4.08881700 \\ \mathrm{H} & -1.37963600 & 0.58847900 & 4.52723600 \\ \mathrm{H} & -1.37963600 & 0.58847900 & -4.52723600 \\ \mathrm{H} & -1.42525700 & -1.12264000 & -4.08881700\end{array}$

Compound 2c: $\quad \mathrm{E}(\mathrm{RB} 3 \mathrm{LYP})=-929.01832661$ a.u.

$\begin{array}{lrrr}\mathrm{C} & -0.00034900 & 0.71197600 & -1.60489300 \\ \mathrm{C} & -0.00002800 & 3.21576800 & -0.36185700 \\ \mathrm{C} & 1.23274300 & 1.41158000 & -1.43874800 \\ \mathrm{C} & -1.23295400 & 1.40752700 & -1.43699000 \\ \mathrm{C} & -1.20359800 & 2.66560500 & -0.81736200 \\ \mathrm{C} & 1.20488700 & 2.67120600 & -0.82553600 \\ \mathrm{H} & -2.14544300 & 3.12756800 & -0.52707100 \\ \mathrm{H} & 2.14788800 & 3.13547500 & -0.54161100 \\ \mathrm{C} & 0.00034900 & -0.71197600 & -1.60489300 \\ \mathrm{C} & 0.00002800 & -3.21576800 & -0.36185700 \\ \mathrm{C} & 1.23295400 & -1.40752700 & -1.43699000 \\ \mathrm{C} & -1.23274300 & -1.41158000 & -1.43874800 \\ \mathrm{C} & -1.20488700 & -2.67120600 & -0.82553600 \\ \mathrm{C} & 1.20359800 & -2.66560500 & -0.81736200 \\ \mathrm{H} & -2.14788800 & -3.13547500 & -0.54161100 \\ \mathrm{H} & 2.14544300 & -3.12756800 & -0.52707100 \\ \mathrm{C} & -2.46232300 & -0.68268600 & -1.63074600 \\ \mathrm{H} & -3.39835700 & -1.23552200 & -1.65811600 \\ \mathrm{C} & -2.46232300 & 0.67838500 & -1.62754400 \\ \mathrm{H} & -3.39854200 & 1.23108300 & -1.65225400 \\ \mathrm{C} & 2.46232300 & -0.67838500 & -1.62754400 \\ \mathrm{H} & 3.39854200 & -1.23108300 & -1.65225400 \\ \mathrm{C} & 2.46232300 & 0.68268600 & -1.63074600 \\ \mathrm{H} & 3.39835700 & 1.23552200 & -1.65811600 \\ \mathrm{C} & -0.00249500 & -4.10130100 & 0.86322000 \\ \mathrm{C} & 0.00249500 & 4.10130100 & 0.86322000 \\ \mathrm{C} & -0.39345500 & -3.25676300 & 2.12444400 \\ \mathrm{H} & -1.45613500 & -2.99878400 & 2.03039100 \\ \mathrm{C} & 0.41725100 & 0.64969900 & 2.36077900\end{array}$




$\begin{array}{lrrr}\mathrm{H} & 1.05367700 & 0.65196900 & 1.46614900 \\ \mathrm{C} & -0.42409100 & 1.95073400 & 2.37921700 \\ \mathrm{H} & 1.10223200 & 0.65168200 & 3.22224700 \\ \mathrm{C} & 0.42409100 & -1.95073400 & 2.37921700 \\ \mathrm{H} & 1.21786000 & -1.85835400 & 1.62895700 \\ \mathrm{H} & 0.93682200 & -2.02602400 & 3.34734000 \\ \mathrm{C} & -0.41725100 & -0.64969900 & 2.36077900 \\ \mathrm{H} & -1.05367700 & -0.65196900 & 1.46614900 \\ \mathrm{H} & -1.10223200 & -0.65168200 & 3.22224700 \\ \mathrm{H} & -0.93682200 & 2.02602400 & 3.34734000 \\ \mathrm{H} & -1.21786000 & 1.85835400 & 1.62895700 \\ \mathrm{C} & 0.39345500 & 3.25676300 & 2.12444400 \\ \mathrm{H} & 1.45613500 & 2.99878400 & 2.03039100 \\ \mathrm{H} & 0.32263600 & 3.91915600 & 2.99687000 \\ \mathrm{H} & -0.32263600 & -3.91915600 & 2.99687000 \\ \mathrm{H} & -0.71820600 & -4.92924600 & 0.77345700 \\ \mathrm{H} & 0.98948400 & -4.54665700 & 1.00973400 \\ \mathrm{H} & 0.71820600 & 4.92924600 & 0.77345700 \\ \mathrm{H} & -0.98948400 & 4.54665700 & 1.00973400\end{array}$

Compound 2d: $\quad \mathrm{E}(\mathrm{RB} 3 \mathrm{LYP})=-968.35170567$ a.u.

$\begin{array}{lrrr}\mathrm{C} & 1.58272200 & 0.13305300 & 0.71181300 \\ \mathrm{C} & 0.57544600 & 0.02790300 & 3.32813200 \\ \mathrm{C} & 1.58774500 & -1.10645000 & 1.41976900 \\ \mathrm{C} & 1.31610700 & 1.34540900 & 1.41269900 \\ \mathrm{C} & 0.82148100 & 1.26361000 & 2.72161400 \\ \mathrm{C} & 1.09076900 & -1.13052100 & 2.73087100 \\ \mathrm{H} & 0.48929400 & 2.17552900 & 3.21392800 \\ \mathrm{H} & 0.96268600 & -2.09167800 & 3.22590900 \\ \mathrm{C} & 1.58272200 & 0.13305300 & -0.71181300 \\ \mathrm{C} & 0.57544600 & 0.02790300 & -3.32813200 \\ \mathrm{C} & 1.58774500 & -1.10645000 & -1.41976900 \\ \mathrm{C} & 1.31610700 & 1.34540900 & -1.41269900 \\ \mathrm{C} & 0.82148100 & 1.26361000 & -2.72161400 \\ \mathrm{C} & 1.09076900 & -1.13052100 & -2.73087100 \\ \mathrm{H} & 0.48929400 & 2.17552900 & -3.21392800 \\ \mathrm{H} & 0.96268600 & -2.09167800 & -3.22590900 \\ \mathrm{C} & 1.34148400 & 2.58486300 & -0.68059900 \\ \mathrm{H} & 1.26268500 & 3.51780500 & -1.23371200 \\ \mathrm{C} & 1.34148400 & 2.58486300 & 0.68059900 \\ \mathrm{H} & 1.26268500 & 3.51780500 & 1.23371200 \\ \mathrm{C} & 1.87774400 & -2.30988500 & -0.68086800 \\ \mathrm{H} & 2.00185400 & -3.23969200 & -1.23105500 \\ \mathrm{C} & 1.87774400 & -2.30988500 & 0.68086800\end{array}$




$\begin{array}{lrrr}\mathrm{H} & 2.00185400 & -3.23969200 & 1.23105500 \\ \mathrm{C} & -0.45864500 & -0.06640100 & -4.42533300 \\ \mathrm{C} & -0.45864500 & -0.06640100 & 4.42533300 \\ \mathrm{C} & -2.38480900 & 0.26491500 & -2.61691200 \\ \mathrm{H} & -3.45887000 & 0.45177100 & -2.75122600 \\ \mathrm{C} & -2.17502200 & -0.47914100 & -1.27911100 \\ \mathrm{H} & -2.77329800 & -1.40299800 & -1.29791500 \\ \mathrm{H} & -1.13118500 & -0.80209300 & -1.21251500 \\ \mathrm{C} & -2.38480900 & 0.26491500 & 2.61691200 \\ \mathrm{H} & -3.45887000 & 0.45177100 & 2.75122600 \\ \mathrm{C} & -1.84931500 & -0.50506300 & 3.86054300 \\ \mathrm{H} & -1.91374200 & 1.25517300 & 2.56198700 \\ \mathrm{C} & -2.51343400 & 0.31520900 & 0.00000000 \\ \mathrm{H} & -3.57637700 & 0.60055800 & 0.00000000 \\ \mathrm{H} & -1.94098500 & 1.25410700 & 0.00000000 \\ \mathrm{C} & -2.17502200 & -0.47914100 & 1.27911100 \\ \mathrm{H} & -2.77329800 & -1.40299800 & 1.29791500 \\ \mathrm{H} & -1.13118500 & -0.80209300 & 1.21251500 \\ \mathrm{H} & -1.79948400 & -1.57502000 & 3.61783200 \\ \mathrm{H} & -1.91374200 & 1.25517300 & -2.56198700 \\ \mathrm{C} & -1.84931500 & -0.50506300 & -3.86054300 \\ \mathrm{H} & -2.56855100 & -0.41825100 & -4.68528100 \\ \mathrm{H} & -1.79948400 & -1.57502000 & -3.61783200 \\ \mathrm{H} & -2.56855100 & -0.41825100 & 4.68528100 \\ \mathrm{H} & -0.16258900 & -0.78565500 & 5.20030300 \\ \mathrm{H} & -0.56404500 & 0.90839800 & 4.91807600 \\ \mathrm{H} & -0.56404500 & 0.90839800 & -4.91807600 \\ \mathrm{H} & -0.16258900 & -0.78565500 & -5.20030300\end{array}$

Compound 2e: $\quad$ E(RB3LYP $)=-1007.67786298$ a.u.

$\begin{array}{lrrr}\mathrm{C} & -0.13547300 & 0.69863500 & -1.56175100 \\ \mathrm{C} & -0.65387300 & 3.36000700 & -0.79971000 \\ \mathrm{C} & 0.94099700 & 1.62684800 & -1.45559200 \\ \mathrm{C} & -1.48223700 & 1.16561600 & -1.48039700 \\ \mathrm{C} & -1.71273600 & 2.50028000 & -1.11713200 \\ \mathrm{C} & 0.65387300 & 2.94551200 & -1.07374700 \\ \mathrm{H} & -2.73765800 & 2.83063500 & -0.95700400 \\ \mathrm{H} & 1.48003800 & 3.62876800 & -0.88684100 \\ \mathrm{C} & 0.13547300 & -0.69863500 & -1.56175100 \\ \mathrm{C} & 0.65387300 & -3.36000700 & -0.79971000 \\ \mathrm{C} & 1.48223700 & -1.16561600 & -1.48039700 \\ \mathrm{C} & -0.94099700 & -1.62684800 & -1.45559200 \\ \mathrm{C} & -0.65387300 & -2.94551200 & -1.07374700 \\ \mathrm{C} & 1.71273600 & -2.50028000 & -1.11713200\end{array}$




$\begin{array}{lrrr}\mathrm{H} & -1.48003800 & -3.62876800 & -0.88684100 \\ \mathrm{H} & 2.73765800 & -2.83063500 & -0.95700400 \\ \mathrm{C} & -2.28755300 & -1.13344200 & -1.57815900 \\ \mathrm{H} & -3.10339900 & -1.85242400 & -1.58798100 \\ \mathrm{C} & -2.54675800 & 0.20352500 & -1.59699500 \\ \mathrm{H} & -3.57200700 & 0.56516500 & -1.62233400 \\ \mathrm{C} & 2.54675800 & -0.20352500 & -1.59699500 \\ \mathrm{H} & 3.57200700 & -0.56516500 & -1.62233400 \\ \mathrm{C} & 2.28755300 & 1.13344200 & -1.57815900 \\ \mathrm{H} & 3.10339900 & 1.85242400 & -1.58798100 \\ \mathrm{C} & 0.89433500 & -4.60013200 & 0.03102400 \\ \mathrm{C} & -0.89433500 & 4.60013200 & 0.03102400 \\ \mathrm{C} & 0.23524100 & -3.24718200 & 2.20252300 \\ \mathrm{H} & 0.00604300 & -3.59474700 & 3.21851600 \\ \mathrm{C} & 0.78107600 & -1.80234800 & 2.27879800 \\ \mathrm{H} & 1.54692900 & -1.75160200 & 3.06733100 \\ \mathrm{H} & 1.29850700 & -1.56625100 & 1.34269800 \\ \mathrm{C} & -0.78107600 & 1.80234800 & 2.27879800 \\ \mathrm{H} & -1.54692900 & 1.75160200 & 3.06733100 \\ \mathrm{C} & -0.23524100 & 3.24718200 & 2.20252300 \\ \mathrm{H} & -1.29850700 & 1.56625100 & 1.34269800 \\ \mathrm{C} & -0.28530200 & -0.71761100 & 2.51337500 \\ \mathrm{H} & -0.82297300 & -0.90589900 & 3.45452400 \\ \mathrm{H} & -1.03470100 & -0.79839600 & 1.71330400 \\ \mathrm{C} & 0.28530200 & 0.71761100 & 2.51337500 \\ \mathrm{H} & 0.82297300 & 0.90589900 & 3.45452400 \\ \mathrm{H} & 1.03470100 & 0.79839600 & 1.71330400 \\ \mathrm{H} & 0.72325700 & 3.24286600 & 1.66690900 \\ \mathrm{H} & -0.00604300 & 3.59474700 & 3.21851600 \\ \mathrm{C} & -1.20332500 & 4.25489800 & 1.52180600 \\ \mathrm{H} & -1.20972500 & 5.20228900 & 2.07599100 \\ \mathrm{H} & -0.72325700 & -3.24286600 & 1.66690900 \\ \mathrm{C} & 1.20332500 & -4.25489800 & 1.52180600 \\ \mathrm{H} & 1.20972500 & -5.20228900 & 2.07599100 \\ \mathrm{H} & 2.22750200 & -3.86356300 & 1.58517100 \\ \mathrm{H} & -2.22750200 & 3.86356300 & 1.58517100 \\ \mathrm{H} & -0.00561300 & 5.24258500 & -0.00778600 \\ \mathrm{H} & -1.73016100 & 5.19082500 & -0.36676000 \\ & 0.00561300 & -5.24258500 & -0.00778600 \\ & 1.73016100 & -5.19082500 & -0.36676000\end{array}$

Compound 3a: $\quad E(R B 3 L Y P)=-922.10440831$ a.u.
C
0.01345000
$0.71717200-1.63601500$
C
0.05359000
$\begin{array}{ll}2.70161100 & 0.25089500\end{array}$ 


$\begin{array}{lrrr}\mathrm{C} & 1.25647100 & 1.35437200 & -1.34371700 \\ \mathrm{C} & -1.20475500 & 1.39795600 & -1.33865300 \\ \mathrm{C} & -1.16241300 & 2.42397800 & -0.38409300 \\ \mathrm{C} & 1.25826300 & 2.37072400 & -0.37543700 \\ \mathrm{H} & -2.08883800 & 2.79780100 & 0.04391400 \\ \mathrm{H} & 2.19417100 & 2.68744500 & 0.07649300 \\ \mathrm{C} & -0.01345000 & -0.71717200 & -1.63601500 \\ \mathrm{C} & -0.05359000 & -2.70161100 & 0.25089500 \\ \mathrm{C} & 1.20475500 & -1.39795600 & -1.33865300 \\ \mathrm{C} & -1.25647100 & -1.35437200 & -1.34371700 \\ \mathrm{C} & -1.25826300 & -2.37072400 & -0.37543700 \\ \mathrm{C} & 1.16241300 & -2.42397800 & -0.38409300 \\ \mathrm{H} & -2.19417100 & -2.68744500 & 0.07649300 \\ \mathrm{H} & 2.08883800 & -2.79780100 & 0.04391400 \\ \mathrm{C} & -2.47646100 & -0.63254900 & -1.65574200 \\ \mathrm{H} & -3.41589100 & -1.17562300 & -1.72210300 \\ \mathrm{C} & -2.45114500 & 0.72515200 & -1.65385900 \\ \mathrm{H} & -3.36955700 & 1.30327300 & -1.71896700 \\ \mathrm{C} & 2.45114500 & -0.72515200 & -1.65385900 \\ \mathrm{H} & 3.36955700 & -1.30327300 & -1.71896700 \\ \mathrm{C} & 2.47646100 & 0.63254900 & -1.65574200 \\ \mathrm{H} & 3.41589100 & 1.17562300 & -1.72210300 \\ \mathrm{O} & -0.07329400 & -3.05653700 & 1.58869700 \\ \mathrm{O} & 0.07329400 & 3.05653700 & 1.58869700 \\ \mathrm{C} & 0.42367600 & -1.96387400 & 2.42831100 \\ \mathrm{H} & 1.45511500 & -1.74115200 & 2.13022200 \\ \mathrm{H} & 0.45175400 & -2.40349400 & 3.42843300 \\ \mathrm{C} & -0.42367600 & -0.65267700 & 2.41743600 \\ \mathrm{H} & -1.05416400 & -0.64704800 & 1.52284200 \\ \mathrm{H} & -1.10901900 & -0.66045200 & 3.27483500 \\ \mathrm{C} & 0.42367600 & 0.65267700 & 2.41743600 \\ \mathrm{H} & 1.05416400 & 0.64704800 & 1.52284200 \\ \mathrm{H} & 1.10901900 & 0.66045200 & 3.27483500 \\ \mathrm{C} & -0.42367600 & 1.96387400 & 2.42831100 \\ \mathrm{H} & -0.45175400 & 2.40349400 & 3.42843300 \\ & -1.45511500 & 1.74115200 & 2.13022200\end{array}$

Compound 3b: $\quad \mathrm{E}(\mathrm{RB} 3 \mathrm{LYP})=-961.44975577$ a.u.

$\begin{array}{lrrr}\mathrm{C} & 0.00347300 & -0.02786000 & 2.91206300 \\ \mathrm{H} & 0.07173600 & 2.12371800 & 2.93402800 \\ \mathrm{C} & 0.50425500 & 1.21679900 & 2.51997800 \\ \mathrm{C} & 1.48064800 & -1.17228300 & 1.38906800 \\ \mathrm{C} & 1.33137100 & 1.28939900 & 1.39022200 \\ \mathrm{C} & 0.63984200 & -1.20351600 & 2.51042500\end{array}$




$\begin{array}{lrrr}\mathrm{C} & 1.65261400 & 0.07382100 & 0.71490700 \\ \mathrm{C} & 1.52591300 & 2.53462500 & 0.67959500 \\ \mathrm{H} & 0.29662100 & -2.15288100 & 2.91173000 \\ \mathrm{C} & 1.52591300 & 2.53462500 & -0.67959500 \\ \mathrm{H} & 1.51768600 & 3.46834300 & 1.23652100 \\ \mathrm{H} & 1.51768600 & 3.46834300 & -1.23652100 \\ \mathrm{C} & 1.33137100 & 1.28939900 & -1.39022200 \\ \mathrm{C} & 0.50425500 & 1.21679900 & -2.51997800 \\ \mathrm{C} & 1.65261400 & 0.07382100 & -0.71490700 \\ \mathrm{C} & 0.00347300 & -0.02786000 & -2.91206300 \\ \mathrm{H} & 0.07173600 & 2.12371800 & -2.93402800 \\ \mathrm{C} & 0.63984200 & -1.20351600 & -2.51042500 \\ \mathrm{H} & 0.29662100 & -2.15288100 & -2.91173000 \\ \mathrm{C} & 1.48064800 & -1.17228300 & -1.38906800 \\ \mathrm{C} & 1.82330100 & -2.38535200 & 0.67945900 \\ \mathrm{H} & 1.92700700 & -3.31274400 & 1.23728200 \\ \mathrm{C} & 1.82330100 & -2.38535200 & -0.67945900 \\ \mathrm{H} & 1.92700700 & -3.31274400 & -1.23728200 \\ \mathrm{O} & -1.22633200 & -0.12530200 & -3.53070200 \\ \mathrm{O} & -1.22633200 & -0.12530200 & 3.53070200 \\ \mathrm{C} & -2.31831600 & 0.25384300 & -2.64197800 \\ \mathrm{H} & -3.19986500 & 0.09256000 & -3.26720300 \\ \mathrm{H} & -2.24970900 & 1.32978300 & -2.43370600 \\ \mathrm{C} & -2.46307100 & -0.54045800 & -1.30322800 \\ \mathrm{H} & -1.85951600 & -1.45333400 & -1.36480900 \\ \mathrm{H} & -3.50724100 & -0.87707200 & -1.23892100 \\ \mathrm{C} & -2.31831600 & 0.25384300 & 2.64197800 \\ \mathrm{H} & -2.24970900 & 1.32978300 & 2.43370600 \\ \mathrm{H} & -3.19986500 & 0.09256000 & 3.26720300 \\ \mathrm{C} & -2.46307100 & -0.54045800 & 1.30322800 \\ \mathrm{H} & -3.50724100 & -0.87707200 & 1.23892100 \\ \mathrm{H} & -1.85951600 & -1.45333400 & 1.36480900 \\ \mathrm{C} & -2.12715600 & 0.23435500 & 0.00000000 \\ \mathrm{H} & -2.69827100 & 1.17509400 & 0.00000000 \\ \mathrm{H} & -1.07127800 & 0.51866500 & 0.00000000\end{array}$

Compound 3c: $\quad \mathrm{E}(\mathrm{RB} 3 \mathrm{LYP})=-1000.79019555$ a.u.

$\begin{array}{lrrr}\mathrm{C} & 0.00125700 & 0.71265200 & -1.62089100 \\ \mathrm{C} & -0.00125700 & 3.11937900 & -0.26176700 \\ \mathrm{C} & 1.23628500 & 1.40226500 & -1.42563200 \\ \mathrm{C} & -1.23256800 & 1.40662600 & -1.43897700 \\ \mathrm{C} & -1.21157900 & 2.63202100 & -0.75824900 \\ \mathrm{C} & 1.21361400 & 2.62918100 & -0.74718100 \\ \mathrm{H} & -2.13794400 & 3.09483200 & -0.42992400\end{array}$




$\begin{array}{lrrr}\mathrm{H} & 2.14371800 & 3.09028100 & -0.42578500 \\ \mathrm{C} & -0.00125700 & -0.71265200 & -1.62089100 \\ \mathrm{C} & 0.00125700 & -3.11937900 & -0.26176700 \\ \mathrm{C} & 1.23256800 & -1.40662600 & -1.43897700 \\ \mathrm{C} & -1.23628500 & -1.40226500 & -1.42563200 \\ \mathrm{C} & -1.21361400 & -2.62918100 & -0.74718100 \\ \mathrm{C} & 1.21157900 & -2.63202100 & -0.75824900 \\ \mathrm{H} & -2.14371800 & -3.09028100 & -0.42578500 \\ \mathrm{H} & 2.13794400 & -3.09483200 & -0.42992400 \\ \mathrm{C} & -2.46494600 & -0.67763000 & -1.64287900 \\ \mathrm{H} & -3.40073400 & -1.23038100 & -1.67334200 \\ \mathrm{C} & -2.46293700 & 0.68284700 & -1.65090000 \\ \mathrm{H} & -3.39695400 & 1.23805900 & -1.68813400 \\ \mathrm{C} & 2.46293700 & -0.68284700 & -1.65090000 \\ \mathrm{H} & 3.39695400 & -1.23805900 & -1.68813400 \\ \mathrm{C} & 2.46494600 & 0.67763000 & -1.64287900 \\ \mathrm{H} & 3.40073400 & 1.23038100 & -1.67334200 \\ \mathrm{O} & 0.03312100 & -3.95651900 & 0.83375700 \\ \mathrm{O} & -0.03312100 & 3.95651900 & 0.83375700 \\ \mathrm{C} & -0.36574200 & -3.27037800 & 2.05763400 \\ \mathrm{H} & -1.44658400 & -3.07818000 & 2.01952400 \\ \mathrm{C} & 0.42574000 & 0.64587100 & 2.28172700 \\ \mathrm{H} & 1.02626500 & 0.66433100 & 1.36434500 \\ \mathrm{C} & -0.40170600 & 1.95436500 & 2.38692600 \\ \mathrm{H} & 1.14327400 & 0.61391600 & 3.11518400 \\ \mathrm{C} & 0.40170600 & -1.95436500 & 2.38692600 \\ \mathrm{H} & 1.27859900 & -1.89102700 & 1.73345300 \\ \mathrm{H} & 0.79828300 & -2.03573400 & 3.40757500 \\ \mathrm{C} & -0.42574000 & -0.64587100 & 2.28172700 \\ \mathrm{H} & -1.02626500 & -0.66433100 & 1.36434500 \\ \mathrm{H} & -1.14327400 & -0.61391600 & 3.11518400 \\ \mathrm{H} & -0.79828300 & 2.03573400 & 3.40757500 \\ \mathrm{H} & -1.27859900 & 1.89102700 & 1.73345300 \\ \mathrm{C} & 0.36574200 & 3.27037800 & 2.05763400 \\ \mathrm{H} & 1.44658400 & 3.07818000 & 2.01952400 \\ \mathrm{H} & 0.18876500 & 4.02472900 & 2.82817400 \\ \mathrm{H} & -0.18876500 & -4.02472900 & 2.82817400\end{array}$

Compound 3d: $\quad E(R B 3 L Y P)=-1040.12647949$ a.u.

$\begin{array}{lrrr}\mathrm{C} & -1.60410600 & -0.05118400 & 0.71243800 \\ \mathrm{C} & -0.48860200 & -0.01783500 & 3.25553900 \\ \mathrm{C} & -1.50051900 & 1.18694900 & 1.41675500 \\ \mathrm{C} & -1.41102000 & -1.28173000 & 1.41046200 \\ \mathrm{C} & -0.85445700 & -1.24206500 & 2.69518200\end{array}$




\begin{tabular}{|c|c|c|c|}
\hline $\mathrm{C}$ & -0.95176100 & 1.18165900 & 2.70876000 \\
\hline $\mathrm{H}$ & -0.54953500 & -2.15810800 & 3.19280200 \\
\hline $\mathrm{H}$ & -0.72721100 & 2.11962400 & 3.20947200 \\
\hline $\mathrm{C}$ & -1.60410600 & -0.05118400 & -0.71243800 \\
\hline $\mathrm{C}$ & -0.48860200 & -0.01783500 & -3.25553900 \\
\hline $\mathrm{C}$ & -1.50051900 & 1.18694900 & -1.41675500 \\
\hline $\mathrm{C}$ & -1.41102000 & -1.28173000 & -1.41046200 \\
\hline $\mathrm{C}$ & -0.85445700 & -1.24206500 & -2.69518200 \\
\hline $\mathrm{C}$ & -0.95176100 & 1.18165900 & -2.70876000 \\
\hline $\mathrm{H}$ & -0.54953500 & -2.15810800 & -3.19280200 \\
\hline $\mathrm{H}$ & -0.72721100 & 2.11962400 & -3.20947200 \\
\hline $\mathrm{C}$ & -1.54533000 & -2.51662400 & -0.68015700 \\
\hline $\mathrm{H}$ & -1.54107800 & -3.45216900 & -1.23401400 \\
\hline $\mathrm{C}$ & -1.54533000 & -2.51662400 & 0.68015700 \\
\hline $\mathrm{H}$ & -1.54107800 & -3.45216900 & 1.23401400 \\
\hline $\mathrm{C}$ & -1.71702000 & 2.40741500 & -0.68092000 \\
\hline $\mathrm{H}$ & -1.77533100 & 3.34339100 & -1.23120900 \\
\hline $\mathrm{C}$ & -1.71702000 & 2.40741500 & 0.68092000 \\
\hline $\mathrm{H}$ & -1.77533100 & 3.34339100 & 1.23120900 \\
\hline $\mathrm{O}$ & 0.43494300 & -0.02296600 & -4.27884000 \\
\hline $\mathrm{O}$ & 0.43494300 & -0.02296600 & 4.27884000 \\
\hline $\mathrm{C}$ & 2.35463900 & -0.34196200 & -2.61984400 \\
\hline $\mathrm{H}$ & 3.42832300 & -0.48557100 & -2.80455900 \\
\hline $\mathrm{C}$ & 2.17036000 & 0.39868300 & -1.27521100 \\
\hline $\mathrm{H}$ & 2.76013700 & 1.32728600 & -1.30557800 \\
\hline $\mathrm{H}$ & 1.12776600 & 0.71262600 & -1.18013600 \\
\hline $\mathrm{C}$ & 2.35463900 & -0.34196200 & 2.61984400 \\
\hline $\mathrm{H}$ & 3.42832300 & -0.48557100 & 2.80455900 \\
\hline $\mathrm{C}$ & 1.76614600 & 0.38448800 & 3.85894600 \\
\hline $\mathrm{H}$ & 1.92133800 & -1.34842200 & 2.56799500 \\
\hline $\mathrm{C}$ & 2.53902700 & -0.39143800 & 0.00000000 \\
\hline $\mathrm{H}$ & 3.60910900 & -0.64663500 & 0.00000000 \\
\hline $\mathrm{H}$ & 1.99264300 & -1.34527700 & 0.00000000 \\
\hline $\mathrm{C}$ & 2.17036000 & 0.39868300 & 1.27521100 \\
\hline $\mathrm{H}$ & 2.76013700 & 1.32728600 & 1.30557800 \\
\hline $\mathrm{H}$ & 1.12776600 & 0.71262600 & 1.18013600 \\
\hline $\mathrm{H}$ & 1.77399600 & 1.47158400 & 3.69494800 \\
\hline $\mathrm{H}$ & 1.92133800 & -1.34842200 & -2.56799500 \\
\hline $\mathrm{C}$ & 1.76614600 & 0.38448800 & -3.85894600 \\
\hline $\mathrm{H}$ & 2.37412100 & 0.17699400 & -4.74333700 \\
\hline $\mathrm{H}$ & 1.77399600 & 1.47158400 & -3.69494800 \\
\hline $\mathrm{H}$ & 2.37412100 & 0.17699400 & 4.74333700 \\
\hline
\end{tabular}


Compound 3e: $\quad E(R B 3 L Y P)=-1079.45472186$ a.u.

$\begin{array}{lrrr}\mathrm{C} & -0.01867300 & -0.71157100 & -1.57434000 \\ \mathrm{C} & -0.08776800 & -3.36444100 & -0.71518500 \\ \mathrm{C} & -1.27241000 & -1.38456100 & -1.45995600 \\ \mathrm{C} & 1.19700600 & -1.45509400 & -1.47249200 \\ \mathrm{C} & 1.13977300 & -2.79469300 & -1.06092200 \\ \mathrm{C} & -1.28422700 & -2.71825600 & -1.02861500 \\ \mathrm{H} & 2.05699800 & -3.34740900 & -0.87619200 \\ \mathrm{H} & -2.22253800 & -3.22020200 & -0.81097100 \\ \mathrm{C} & 0.01867300 & 0.71157100 & -1.57434000 \\ \mathrm{C} & 0.08776800 & 3.36444100 & -0.71518500 \\ \mathrm{C} & -1.19700600 & 1.45509400 & -1.47249200 \\ \mathrm{C} & 1.27241000 & 1.38456100 & -1.45995600 \\ \mathrm{C} & 1.28422700 & 2.71825600 & -1.02861500 \\ \mathrm{C} & -1.13977300 & 2.79469300 & -1.06092200 \\ \mathrm{H} & 2.22253800 & 3.22020200 & -0.81097100 \\ \mathrm{H} & -2.05699800 & 3.34740900 & -0.87619200 \\ \mathrm{C} & 2.48018300 & 0.61299800 & -1.59804000 \\ \mathrm{H} & 3.43161400 & 1.13890900 & -1.61673300 \\ \mathrm{C} & 2.44392300 & -0.74811300 & -1.60808600 \\ \mathrm{H} & 3.36623200 & -1.32336500 & -1.63549400 \\ \mathrm{C} & -2.44392300 & 0.74811300 & -1.60808600 \\ \mathrm{H} & -3.36623200 & 1.32336500 & -1.63549400 \\ \mathrm{C} & -2.48018300 & -0.61299800 & -1.59804000 \\ \mathrm{H} & -3.43161400 & -1.13890900 & -1.61673300 \\ \mathrm{O} & 0.14834700 & 4.52981700 & 0.01759000 \\ \mathrm{O} & -0.14834700 & -4.52981700 & 0.01759000 \\ \mathrm{C} & 0.39815200 & 3.23098500 & 2.18140300 \\ \mathrm{H} & 0.60519600 & 3.58972200 & 3.19842400 \\ \mathrm{C} & -0.41847900 & 1.91739600 & 2.26232700 \\ \mathrm{H} & -1.18637500 & 2.02128300 & 3.04297200 \\ \mathrm{H} & -0.96274800 & 1.77112800 & 1.32441700 \\ \mathrm{C} & 0.41847900 & -1.91739600 & 2.26232700 \\ \mathrm{H} & 1.18637500 & -2.02128300 & 3.04297200 \\ \mathrm{C} & -0.39815200 & -3.23098500 & 2.18140300 \\ \mathrm{H} & 0.96274800 & -1.77112800 & 1.32441700 \\ \mathrm{C} & 0.41847900 & 0.64976600 & 2.51706600 \\ \mathrm{H} & 0.97227200 & 0.73864700 & 3.46310100 \\ \mathrm{H} & 1.17726000 & 0.58014600 & 1.72497800 \\ \mathrm{C} & -0.41847900 & -0.64976600 & 2.51706600 \\ \mathrm{H} & -0.97227200 & -0.73864700 & 3.46310100 \\ \mathrm{H} & -1.17726000 & -0.58014600 & 1.72497800 \\ \mathrm{H} & -1.37871300 & -3.04185500 & 1.72758900 \\ \mathrm{H} & -0.60519600 & -3.58972200 & 3.19842400 \\ \mathrm{C} & 0.27654700 & -4.38332700 & 1.39796300\end{array}$




$\begin{array}{lrrr}\mathrm{H} & 0.02132000 & -5.35092900 & 1.83778700 \\ \mathrm{H} & 1.37871300 & 3.04185500 & 1.72758900 \\ \mathrm{C} & -0.27654700 & 4.38332700 & 1.39796300 \\ \mathrm{H} & -0.02132000 & 5.35092900 & 1.83778700 \\ \mathrm{H} & -1.37049800 & 4.27827600 & 1.43266500 \\ \mathrm{H} & 1.37049800 & -4.27827600 & 1.43266500\end{array}$

Compound 7a: $\quad \mathrm{E}(\mathrm{RB} 3 \mathrm{LYP})=-3003.55455908$ a.u.

$\begin{array}{lrrr}\mathrm{C} & -1.30418600 & 4.45010300 & 1.29259900 \\ \mathrm{C} & -2.29440200 & 3.41921000 & -1.13115700 \\ \mathrm{C} & -0.67046200 & 4.75909200 & 0.05605600 \\ \mathrm{C} & -2.28241900 & 3.44971700 & 1.27707300 \\ \mathrm{C} & -2.59102700 & 2.78363300 & 0.08175300 \\ \mathrm{C} & -1.33335300 & 4.43839000 & -1.16604800 \\ \mathrm{H} & -2.60365200 & 3.01920600 & 2.22278400 \\ \mathrm{H} & -2.62923900 & 2.98084800 & -2.06730000 \\ \mathrm{C} & 0.76511600 & 4.76658400 & 0.03679200 \\ \mathrm{C} & 2.68399300 & 2.79454400 & -0.03847700 \\ \mathrm{C} & 1.42882200 & 4.41758100 & 1.25086600 \\ \mathrm{C} & 1.40164900 & 4.49350000 & -1.20665900 \\ \mathrm{C} & 2.38133600 & 3.49346100 & -1.21640200 \\ \mathrm{C} & 2.38981400 & 3.39995000 & 1.19041600 \\ \mathrm{H} & 2.69819900 & 3.08402100 & -2.17287200 \\ \mathrm{H} & 2.72141800 & 2.93522500 & 2.11506700 \\ \mathrm{C} & -0.59422800 & 4.76111500 & 2.52215200 \\ \mathrm{H} & -1.14408700 & 4.83215300 & 3.45739600 \\ \mathrm{C} & 0.76169700 & 4.73426400 & 2.50235000 \\ \mathrm{H} & 1.34067500 & 4.78325700 & 3.42128200 \\ \mathrm{C} & -0.66756900 & 4.78940400 & -2.40887100 \\ \mathrm{H} & -1.24645000 & 4.85557300 & -3.32679900 \\ \mathrm{C} & 0.68819800 & 4.82843700 & -2.42804800 \\ \mathrm{H} & 1.23674800 & 4.92535100 & -3.36172200 \\ \mathrm{C} & 2.96903700 & 1.32824000 & -0.15915700 \\ \mathrm{C} & 3.58612900 & -1.33765300 & -0.77990300 \\ \mathrm{C} & 4.26222000 & 0.83123000 & 0.07974200 \\ \mathrm{C} & 1.91315800 & 0.46761700 & -0.59902300 \\ \mathrm{C} & 2.27758200 & -0.84354000 & -1.00681000 \\ \mathrm{C} & 4.56355400 & -0.51937300 & -0.18018900 \\ \mathrm{C} & -2.91294700 & 1.32339300 & 0.17223900 \\ \mathrm{C} & -3.62136600 & -1.32545000 & 0.76880900 \\ \mathrm{C} & -1.89055000 & 0.42889700 & 0.62239800 \\ \mathrm{C} & -4.21540500 & 0.86575000 & -0.09414700 \\ \mathrm{C} & -4.56187200 & -0.47650400 & 0.15341100 \\ \mathrm{C} & -2.30225500 & -0.87205100 & 1.02002200 \\ & & & \\ & & & \end{array}$




\begin{tabular}{|c|c|c|c|}
\hline $\mathrm{C}$ & -0.38288200 & 0.79802400 & 0.71113300 \\
\hline $\mathrm{H}$ & -0.25293100 & 1.74257900 & 1.24423900 \\
\hline $\mathrm{H}$ & 0.10354800 & 0.03620500 & 1.31926300 \\
\hline $\mathrm{C}$ & 0.41805900 & 0.88730600 & -0.65620500 \\
\hline $\mathrm{H}$ & 0.32506200 & 1.90212500 & -1.04474600 \\
\hline $\mathrm{H}$ & -0.08460000 & 0.23757300 & -1.37226600 \\
\hline $\mathrm{C}$ & 5.32724300 & 1.77026600 & 0.56675800 \\
\hline $\mathrm{C}$ & 7.30426800 & 3.55772800 & 1.47168300 \\
\hline $\mathrm{C}$ & 5.65977700 & 1.84275400 & 1.92651800 \\
\hline $\mathrm{C}$ & 6.00225500 & 2.60781500 & -0.33201600 \\
\hline $\mathrm{C}$ & 6.98331300 & 3.49326500 & 0.11492900 \\
\hline $\mathrm{C}$ & 6.63893100 & 2.72947600 & 2.37657200 \\
\hline $\mathrm{H}$ & 5.14560400 & 1.19923400 & 2.63537600 \\
\hline $\mathrm{H}$ & 5.75416700 & 2.56346600 & -1.38907000 \\
\hline $\mathrm{H}$ & 7.49631400 & 4.13374600 & -0.59790900 \\
\hline $\mathrm{H}$ & 6.88207800 & 2.77179000 & 3.43522700 \\
\hline $\mathrm{H}$ & 8.06754600 & 4.24798300 & 1.82109600 \\
\hline $\mathrm{C}$ & 5.94474200 & -1.04256000 & 0.08646500 \\
\hline $\mathrm{C}$ & 8.54070000 & -2.00434900 & 0.59435000 \\
\hline $\mathrm{C}$ & 6.29575300 & -1.52407100 & 1.35514300 \\
\hline $\mathrm{C}$ & 6.91610300 & -1.05091900 & -0.92360800 \\
\hline $\mathrm{C}$ & 8.20312900 & -1.52716700 & -0.67293400 \\
\hline $\mathrm{C}$ & 7.58187100 & -2.00209400 & 1.60835300 \\
\hline $\mathrm{H}$ & 5.55225000 & -1.52607100 & 2.14810900 \\
\hline $\mathrm{H}$ & 6.65836800 & -0.68273500 & -1.91275400 \\
\hline $\mathrm{H}$ & 8.94265600 & -1.52516300 & -1.46958700 \\
\hline $\mathrm{H}$ & 7.83419000 & -2.37221100 & 2.59886400 \\
\hline $\mathrm{H}$ & 9.54326600 & -2.37549600 & 0.79007800 \\
\hline $\mathrm{C}$ & 3.97308500 & -2.72236900 & -1.21580400 \\
\hline $\mathrm{C}$ & 4.74047700 & -5.30382700 & -2.03596100 \\
\hline $\mathrm{C}$ & 4.13137400 & -3.75460500 & -0.28063700 \\
\hline $\mathrm{C}$ & 4.20913200 & -3.00477900 & -2.56871600 \\
\hline $\mathrm{C}$ & 4.58987400 & -4.28321900 & -2.97614800 \\
\hline $\mathrm{C}$ & 4.50911700 & -5.03507500 & -0.68620000 \\
\hline $\mathrm{H}$ & 3.95485800 & -3.55050800 & 0.77175600 \\
\hline $\mathrm{H}$ & 4.08697000 & -2.21627100 & -3.30612300 \\
\hline $\mathrm{H}$ & 4.76857600 & -4.48157600 & -4.02989000 \\
\hline $\mathrm{H}$ & 4.62500900 & -5.82224400 & 0.05452500 \\
\hline $\mathrm{H}$ & 5.03673200 & -6.30042400 & -2.35269100 \\
\hline $\mathrm{C}$ & 1.33238500 & -1.69559800 & -1.80581600 \\
\hline $\mathrm{C}$ & -0.32073200 & -3.30225200 & -3.43313300 \\
\hline $\mathrm{C}$ & 0.93916100 & -1.26826200 & -3.08620000 \\
\hline $\mathrm{C}$ & 0.88565500 & -2.94684700 & -1.36316600 \\
\hline $\mathrm{C}$ & 0.06779500 & -3.74234100 & -2.16756900 \\
\hline $\mathrm{C}$ & 0.11942200 & -2.05900600 & -3.89080000 \\
\hline $\mathrm{H}$ & 1.28964100 & -0.30685200 & -3.45345900 \\
\hline
\end{tabular}




\begin{tabular}{|c|c|c|c|}
\hline $\mathrm{H}$ & 1.18618100 & -3.30140800 & -0.38420600 \\
\hline $\mathrm{H}$ & -0.26068400 & -4.71301500 & -1.80426400 \\
\hline $\mathrm{H}$ & -0.16819700 & -1.70632200 & -4.87804100 \\
\hline $\mathrm{H}$ & -0.95434900 & -3.92444900 & -4.05975300 \\
\hline $\mathrm{C}$ & -5.24538400 & 1.83880400 & -0.59029000 \\
\hline $\mathrm{C}$ & -7.15583100 & 3.68986600 & -1.51030800 \\
\hline $\mathrm{C}$ & -5.53426600 & 1.95122000 & -1.95728400 \\
\hline $\mathrm{C}$ & -5.93044600 & 2.66848700 & 0.30806700 \\
\hline $\mathrm{C}$ & -6.87841300 & 3.58561500 & -0.14645400 \\
\hline $\mathrm{C}$ & -6.48040800 & 2.86940900 & -2.41484600 \\
\hline $\mathrm{H}$ & -5.01174300 & 1.31410800 & -2.66592500 \\
\hline $\mathrm{H}$ & -5.71600500 & 2.59341100 & 1.37072900 \\
\hline $\mathrm{H}$ & -7.39961200 & 4.21973200 & 0.56612500 \\
\hline $\mathrm{H}$ & -6.68995100 & 2.94236600 & -3.47901000 \\
\hline $\mathrm{H}$ & -7.89325600 & 4.40482200 & -1.86551900 \\
\hline $\mathrm{C}$ & -5.95303400 & -0.95583800 & -0.14188300 \\
\hline $\mathrm{C}$ & -8.56723700 & -1.83397000 & -0.70479000 \\
\hline $\mathrm{C}$ & -6.93455600 & -0.97172500 & 0.85830800 \\
\hline $\mathrm{C}$ & -6.30336600 & -1.38684900 & -1.42882800 \\
\hline $\mathrm{C}$ & -7.59841900 & -1.82381300 & -1.70918400 \\
\hline $\mathrm{C}$ & -8.23071900 & -1.40609400 & 0.58025200 \\
\hline $\mathrm{H}$ & -6.67763100 & -0.64232800 & 1.86120200 \\
\hline $\mathrm{H}$ & -5.55218400 & -1.38233700 & -2.21447900 \\
\hline $\mathrm{H}$ & -7.84994700 & -2.15550400 & -2.71341000 \\
\hline $\mathrm{H}$ & -8.97809200 & -1.41034100 & 1.36953500 \\
\hline $\mathrm{H}$ & -9.57680300 & -2.17295000 & -0.92175100 \\
\hline $\mathrm{C}$ & -4.05819800 & -2.69880100 & 1.19276900 \\
\hline $\mathrm{C}$ & -4.91879000 & -5.25852600 & 1.98831800 \\
\hline $\mathrm{C}$ & -4.30760300 & -2.98456900 & 2.54268700 \\
\hline $\mathrm{C}$ & -4.25105900 & -3.71646800 & 0.24806000 \\
\hline $\mathrm{C}$ & -4.67459800 & -4.98632100 & 0.64149800 \\
\hline $\mathrm{C}$ & -4.73465800 & -4.25211200 & 2.93783800 \\
\hline $\mathrm{H}$ & -4.15893300 & -2.20766300 & 3.28748300 \\
\hline $\mathrm{H}$ & -4.06541300 & -3.50906100 & -0.80208500 \\
\hline $\mathrm{H}$ & -4.81648900 & -5.76234900 & -0.10641600 \\
\hline $\mathrm{H}$ & -4.92299200 & -4.45319400 & 3.98938200 \\
\hline $\mathrm{H}$ & -5.25082000 & -6.24677500 & 2.29558100 \\
\hline $\mathrm{C}$ & -1.39824600 & -1.75314100 & 1.83537000 \\
\hline $\mathrm{C}$ & 0.18501400 & -3.40369300 & 3.48896500 \\
\hline $\mathrm{C}$ & -1.00090900 & -1.33011700 & 3.11587100 \\
\hline $\mathrm{C}$ & -0.99252000 & -3.02365500 & 1.40689800 \\
\hline $\mathrm{C}$ & -0.20837500 & -3.84020700 & 2.22342400 \\
\hline $\mathrm{C}$ & -0.21596700 & -2.14268000 & 3.93348800 \\
\hline $\mathrm{H}$ & -1.31877000 & -0.35350200 & 3.47241100 \\
\hline $\mathrm{H}$ & -1.29876800 & -3.37556200 & 0.42882400 \\
\hline $\mathrm{H}$ & 0.08985200 & -4.82407600 & 1.86973400 \\
\hline
\end{tabular}



$\mathrm{H}$
$0.07580000 \quad-1.79217400$
4.92028500
$\mathrm{H}$
$0.79219000-4.04214600$
4.12532400

Compound 7b: $\quad \mathrm{E}(\mathrm{RB} 3 \mathrm{LYP})=-3042.91074870$ a.u.

$\begin{array}{lrrr}\mathrm{C} & -1.59877700 & -3.93394600 & -0.64779300 \\ \mathrm{C} & -2.50432700 & -2.94382300 & 1.82855800 \\ \mathrm{C} & -0.85162100 & -4.16718900 & 0.54284800 \\ \mathrm{C} & -2.68389000 & -3.05831900 & -0.57355900 \\ \mathrm{C} & -3.00240200 & -2.41733400 & 0.63319300 \\ \mathrm{C} & -1.42090700 & -3.83708100 & 1.80802000 \\ \mathrm{H} & -3.14117600 & -2.70857600 & -1.49536600 \\ \mathrm{H} & -2.81436500 & -2.51784400 & 2.78005300 \\ \mathrm{C} & 0.56927700 & -4.25799000 & 0.44204400 \\ \mathrm{C} & 2.85934600 & -2.71811200 & 0.26351000 \\ \mathrm{C} & 1.16380100 & -4.05706200 & -0.84034100 \\ \mathrm{C} & 1.34827100 & -4.05500300 & 1.61591900 \\ \mathrm{C} & 2.51890200 & -3.29232400 & 1.49478000 \\ \mathrm{C} & 2.31365800 & -3.26308400 & -0.90695400 \\ \mathrm{H} & 3.00739000 & -2.93783900 & 2.39988200 \\ \mathrm{H} & 2.65222400 & -2.90463200 & -1.87526900 \\ \mathrm{C} & -0.99337900 & -4.26607500 & -1.91980600 \\ \mathrm{H} & -1.62030800 & -4.31485600 & -2.80671500 \\ \mathrm{C} & 0.36001300 & -4.31621100 & -2.01567400 \\ \mathrm{H} & 0.85059900 & -4.40491600 & -2.98189000 \\ \mathrm{C} & -0.64298000 & -4.11330200 & 2.99485500 \\ \mathrm{H} & -1.13166900 & -4.08158000 & 3.96574000 \\ \mathrm{C} & 0.70869100 & -4.23369900 & 2.90097400 \\ \mathrm{H} & 1.32475600 & -4.29730600 & 3.79468000 \\ \mathrm{C} & 3.48211100 & -1.35657900 & 0.24616900 \\ \mathrm{C} & 4.64476800 & 1.19905000 & 0.32935200 \\ \mathrm{C} & 4.76512000 & -1.14894400 & -0.29837500 \\ \mathrm{C} & 2.71554500 & -0.27205600 & 0.76903300 \\ \mathrm{C} & 3.35992200 & 0.98554500 & 0.87976900 \\ \mathrm{C} & 5.33647300 & 0.14072100 & -0.29776300 \\ \mathrm{C} & -3.57919300 & -1.03992000 & 0.49413100 \\ \mathrm{H} & -4.54205000 & 1.45107000 & -0.36641400 \\ \mathrm{C} & -2.67039500 & 0.06140700 & 0.44465600 \\ \mathrm{C} & -4.94325200 & -0.88099000 & 0.18988700 \\ \mathrm{C} & -5.44035300 & 0.37650800 & -0.21495200 \\ \mathrm{C} & -3.17083300 & 1.29078000 & -0.04116800 \\ \mathrm{C} & -1.25454900 & -0.00131000 & 1.07662100 \\ \mathrm{C} & -1.12479800 & 0.95084800 & 1.60645200 \\ \mathrm{C} & 1.28748900 & -0.77032400 & 1.85309700 \\ \mathrm{C} & -0.40589900 & 1.28369100 \\ \mathrm{C} & & -1.36855200 & 1.77708900\end{array}$




\begin{tabular}{|c|c|c|c|}
\hline $\mathrm{C}$ & 5.49864200 & -2.31248100 & -0.89567200 \\
\hline $\mathrm{C}$ & 6.84862000 & -4.51263500 & -2.02224100 \\
\hline $\mathrm{C}$ & 5.75220300 & -2.37061500 & -2.27427500 \\
\hline $\mathrm{C}$ & 5.93231600 & -3.37762900 & -0.09246700 \\
\hline $\mathrm{C}$ & 6.60475800 & -4.46597000 & -0.6487760 \\
\hline $\mathrm{C}$ & 6.41840100 & -3.46112400 & -2.8333150 \\
\hline $\mathrm{H}$ & 5.42909600 & -1.55099800 & -2.9096590 \\
\hline $\mathrm{H}$ & 5.74238000 & -3.34963100 & 0.9768600 \\
\hline $\mathrm{H}$ & 6.93635600 & -5.27877900 & -0.0077330 \\
\hline $\mathrm{H}$ & 6.60340500 & -3.48718900 & -3.9041320 \\
\hline $\mathrm{H}$ & 7.37020700 & -5.36121900 & -2.4569900 \\
\hline $\mathrm{C}$ & 6.67957500 & 0.37733600 & -0.9203370 \\
\hline $\mathrm{C}$ & 9.19559100 & 0.82560400 & -2.1040550 \\
\hline $\mathrm{C}$ & 6.80571300 & 1.19613200 & -2.0523410 \\
\hline $\mathrm{C}$ & 7.83756400 & -0.21212100 & -0.3920580 \\
\hline $\mathrm{C}$ & 9.08426100 & 0.01156900 & -0.9757070 \\
\hline $\mathrm{C}$ & 8.05072500 & 1.41596200 & -2.6414560 \\
\hline $\mathrm{H}$ & 5.91965500 & 1.66477600 & -2.4712170 \\
\hline $\mathrm{H}$ & 7.75812200 & -0.85311800 & 0.4812300 \\
\hline $\mathrm{H}$ & 9.96936800 & -0.45278400 & -0.5484440 \\
\hline $\mathrm{H}$ & 8.12572000 & 2.05184700 & -3.5199010 \\
\hline $\mathrm{H}$ & 10.16658600 & 0.99858800 & -2.5606780 \\
\hline $\mathrm{C}$ & 5.29033800 & 2.54874700 & 0.44212000 \\
\hline $\mathrm{C}$ & 6.50678200 & 5.08079200 & 0.64765300 \\
\hline $\mathrm{C}$ & 4.77047900 & 3.65400900 & -0.2467930 \\
\hline $\mathrm{C}$ & 6.43150700 & 2.73572500 & 1.23558200 \\
\hline $\mathrm{C}$ & 7.03309800 & 3.98936300 & 1.34044000 \\
\hline $\mathrm{C}$ & 5.37356300 & 4.90798300 & -0.1482230 \\
\hline $\mathrm{H}$ & 3.88717000 & 3.52646800 & -0.8663070 \\
\hline $\mathrm{H}$ & 6.85042800 & 1.88958100 & 1.7724960 \\
\hline $\mathrm{H}$ & 7.91527400 & 4.11250900 & 1.96345900 \\
\hline $\mathrm{H}$ & 4.95604200 & 5.75084000 & -0.6932330 \\
\hline $\mathrm{H}$ & 6.97626800 & 6.05780800 & 0.72705600 \\
\hline $\mathrm{C}$ & 2.77924400 & 2.06187900 & 1.75714300 \\
\hline $\mathrm{C}$ & 1.79927200 & 4.02387700 & 3.52526100 \\
\hline $\mathrm{C}$ & 3.27345000 & 2.16652200 & 3.06825600 \\
\hline $\mathrm{C}$ & 1.78202800 & 2.95547300 & 1.34932000 \\
\hline $\mathrm{C}$ & 1.29964400 & 3.92980700 & 2.22654700 \\
\hline $\mathrm{C}$ & 2.78932900 & 3.13514400 & 3.94596500 \\
\hline $\mathrm{H}$ & 4.04727200 & 1.47806700 & 3.3971910 \\
\hline $\mathrm{H}$ & 1.37936200 & 2.89693800 & 0.3424890 \\
\hline $\mathrm{H}$ & 0.52839600 & 4.61288800 & 1.8869470 \\
\hline $\mathrm{H}$ & 3.18789900 & 3.19602900 & 4.9555370 \\
\hline $\mathrm{H}$ & 1.41999900 & 4.78354500 & 4.2039360 \\
\hline $\mathrm{C}$ & -5.84775800 & -2.07314400 & 0.2759060 \\
\hline $\mathrm{C}$ & -7.51328200 & -4.33812400 & 0.4470040 \\
\hline
\end{tabular}




$\begin{array}{lccc}\mathrm{C} & -6.08290800 & -2.70194500 & 1.50759900 \\ \mathrm{C} & -6.46421100 & -2.59986500 & -0.86879500 \\ \mathrm{C} & -7.28651100 & -3.72305100 & -0.78536600 \\ \mathrm{C} & -6.91024100 & -3.82154700 & 1.59469100 \\ \mathrm{H} & -5.61742800 & -2.30290000 & 2.40475000 \\ \mathrm{H} & -6.29762000 & -2.12369200 & -1.83063100 \\ \mathrm{H} & -7.75123200 & -4.11747500 & -1.68532800 \\ \mathrm{H} & -7.08229500 & -4.29072400 & 2.56001600 \\ \mathrm{H} & -8.15578100 & -5.21221500 & 0.51241700 \\ \mathrm{C} & -6.90345700 & 0.55180400 & -0.49181000 \\ \mathrm{C} & -9.65793000 & 0.86693900 & -0.98742900 \\ \mathrm{C} & -7.37461300 & 0.81795500 & -1.78586300 \\ \mathrm{C} & -7.83809700 & 0.44445800 & 0.54872000 \\ \mathrm{C} & -9.20209100 & 0.60389100 & 0.30523800 \\ \mathrm{C} & -8.73865800 & 0.97173700 & -2.03254100 \\ \mathrm{H} & -6.66533000 & 0.90792800 & -2.60342600 \\ \mathrm{H} & -7.49039300 & 0.23231900 & 1.55587100 \\ \mathrm{H} & -9.90893600 & 0.51954600 & 1.12671000 \\ \mathrm{H} & -9.08279400 & 1.17518500 & -3.04335900 \\ \mathrm{H} & -10.72079700 & 0.98868200 & -1.17903300 \\ \mathrm{C} & -5.05565900 & 2.77938600 & -0.84394100 \\ \mathrm{C} & -6.03723000 & 5.26386000 & -1.73587200 \\ \mathrm{C} & -4.73895000 & 3.25946400 & -2.12288600 \\ \mathrm{H} & -5.26114400 & 0.62242300 & -0.38316400 \\ \mathrm{C} & -5.87558300 & 3.56523500 & -0.02127800 \\ \mathrm{C} & -6.36035500 & 4.79705900 & -0.46093100 \\ \mathrm{C} & -5.22589300 & 4.48905500 & -2.56631000 \\ \mathrm{H} & -4.10390600 & 2.66483000 & -2.77341600 \\ \mathrm{H} & -6.13762000 & 3.20375200 & 0.96916700 \\ \mathrm{H} & -6.99328700 & 5.39094400 & 0.19351700 \\ \mathrm{H} & -4.96968800 & 4.84188800 & -3.56207000 \\ \mathrm{H} & -6.19023800 & 5.66413200 & -1.11106600 \\ \mathrm{C} & -1.13288300 & 0.34982100 & 2.06286100 \\ \mathrm{C} & -2.26713700 & 2.46895800 & -0.25901600 \\ \mathrm{C} & -0.75913200 & 4.77054900 & -0.86838400 \\ \mathrm{C} & -1.39011000 & 2.48643900 & -1.35474600 \\ \mathrm{H} & -2.35846500 & 3.61897800 & 0.53829600 \\ \mathrm{H} & -1.61686000 & 4.76057800 & 0.23382100 \\ \mathrm{H} & -0.64194400 & 3.62560800 & -1.65834700 \\ \mathrm{H} & -3.03985900 & 3.62736900 & 1.38383200 \\ \mathrm{H} & -1.11994800 & 5.64907200 & 0.85171800 \\ \mathrm{H} & & & -0.36491600\end{array}$




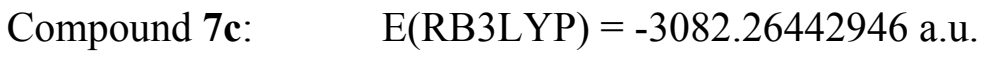

$\begin{array}{lrrr}\mathrm{C} & -1.43510500 & -1.19890200 & -3.54495600 \\ \mathrm{C} & 0.78443900 & -2.77860600 & -2.84447900 \\ \mathrm{C} & -0.11014900 & -0.70380900 & -3.72429400 \\ \mathrm{C} & -1.59999200 & -2.41834400 & -2.87305400 \\ \mathrm{C} & -0.49137200 & -3.10799200 & -2.36837100 \\ \mathrm{C} & 1.00147200 & -1.57590700 & -3.52895600 \\ \mathrm{H} & -2.60055300 & -2.71874700 & -2.56950000 \\ \mathrm{H} & 1.64146700 & -3.36366900 & -2.52101500 \\ \mathrm{C} & 0.11014900 & 0.70380900 & -3.72429400 \\ \mathrm{C} & 0.49137200 & 3.10799200 & -2.36837100 \\ \mathrm{C} & -1.00147200 & 1.57590700 & -3.52895600 \\ \mathrm{C} & 1.43510500 & 1.19890200 & -3.54495600 \\ \mathrm{C} & 1.59999200 & 2.41834400 & -2.87305400 \\ \mathrm{C} & -0.78443900 & 2.77860600 & -2.84447900 \\ \mathrm{H} & 2.60055300 & 2.71874700 & -2.56950000 \\ \mathrm{H} & -1.64146700 & 3.36366900 & -2.52101500 \\ \mathrm{C} & -2.53905400 & -0.29288300 & -3.75361000 \\ \mathrm{H} & -3.54845000 & -0.69584800 & -3.78850400 \\ \mathrm{C} & -2.32911500 & 1.05139700 & -3.74144700 \\ \mathrm{H} & -3.16707400 & 1.74382600 & -3.76607200 \\ \mathrm{C} & 2.32911500 & -1.05139700 & -3.74144700 \\ \mathrm{H} & 3.16707400 & -1.74382600 & -3.76607200 \\ \mathrm{C} & 2.53905400 & 0.29288300 & -3.75361000 \\ \mathrm{H} & 3.54845000 & 0.69584800 & -3.78850400 \\ \mathrm{C} & 0.59989300 & 3.92724800 & -1.11667300 \\ \mathrm{C} & 0.58304400 & 5.37315800 & 1.29428500 \\ \mathrm{C} & 0.43440600 & 5.32440800 & -1.13861100 \\ \mathrm{C} & 0.75675500 & 3.22247800 & 0.11071300 \\ \mathrm{C} & 0.73800100 & 3.96733400 & 1.31120500 \\ \mathrm{C} & 0.43307200 & 6.05671700 & 0.06929700 \\ \mathrm{C} & -0.59989300 & -3.92724800 & -1.11667300 \\ \mathrm{C} & -0.58304400 & -5.37315800 & 1.29428500 \\ \mathrm{C} & -0.75675500 & -3.22247800 & 0.11071300 \\ \mathrm{C} & -0.43440600 & -5.32440800 & -1.13861100 \\ \mathrm{C} & -0.43307200 & -6.05671700 & 0.06929700 \\ \mathrm{C} & -0.73800100 & -3.96733400 & 1.31120500 \\ \mathrm{C} & -1.01517200 & -1.70650200 & 0.16938600 \\ \mathrm{C} & 1.01517200 & 1.70650200 & 0.16938600 \\ \mathrm{C} & 0.23659100 & 6.01274200 & -2.45550400 \\ \mathrm{C} & -0.14483500 & 7.27345600 & -4.94506800 \\ \mathrm{C} & -0.97843800 & 6.64324800 & -2.76295200 \\ \mathrm{C} & 1.25579000 & 6.02446500 & -3.41924000 \\ \mathrm{C} & 1.06953600 & 6.65162600 & -4.65126300 \\ \mathrm{C} & -1.16878400 & 7.26574600 & -3.99640800 \\ & & & \end{array}$




\begin{tabular}{|c|c|c|c|}
\hline $\mathrm{H}$ & -1.77712000 & 6.64815100 & -2.02683100 \\
\hline $\mathrm{H}$ & 2.20344700 & 5.54174000 & -3.19706200 \\
\hline $\mathrm{H}$ & 1.87420800 & 6.65292200 & -5.38205000 \\
\hline $\mathrm{H}$ & -2.11879500 & 7.74638500 & -4.21543600 \\
\hline $\mathrm{H}$ & -0.29216300 & 7.76033700 & -5.90547800 \\
\hline$C$ & 0.27900400 & 7.54797600 & 0.04564900 \\
\hline $\mathrm{C}$ & -0.00911000 & 10.34912300 & -0.01163200 \\
\hline $\mathrm{C}$ & -0.85622200 & 8.16489000 & 0.59164700 \\
\hline $\mathrm{C}$ & 1.26689200 & 8.36059100 & -0.52985500 \\
\hline $\mathrm{C}$ & 1.12671700 & 9.74797100 & -0.55620000 \\
\hline $\mathrm{C}$ & -1.00111000 & 9.55173000 & 0.56139900 \\
\hline $\mathrm{H}$ & -1.62999800 & 7.55142400 & 1.04414900 \\
\hline $\mathrm{H}$ & 2.15007300 & 7.89899300 & -0.96223200 \\
\hline $\mathrm{H}$ & 1.90570200 & 10.35908800 & -1.00486900 \\
\hline $\mathrm{H}$ & -1.89020500 & 10.00916200 & 0.98785800 \\
\hline $\mathrm{H}$ & -0.12041400 & 11.43003000 & -0.03363900 \\
\hline $\mathrm{C}$ & 0.61796000 & 6.14434300 & 2.58156400 \\
\hline $\mathrm{C}$ & 0.69666400 & 7.59766100 & 4.99240700 \\
\hline $\mathrm{C}$ & -0.43307200 & 6.05951200 & 3.50589700 \\
\hline $\mathrm{C}$ & 1.70867500 & 6.97128500 & 2.88666600 \\
\hline $\mathrm{C}$ & 1.75017800 & 7.68953100 & 4.08151000 \\
\hline $\mathrm{C}$ & -0.39641400 & 6.78100400 & 4.69917700 \\
\hline $\mathrm{H}$ & -1.28588800 & 5.42359500 & 3.28604800 \\
\hline $\mathrm{H}$ & 2.52824700 & 7.05319300 & 2.17828000 \\
\hline $\mathrm{H}$ & 2.60603100 & 8.32337700 & 4.29907300 \\
\hline $\mathrm{H}$ & -1.22315300 & 6.70332000 & 5.40070600 \\
\hline $\mathrm{H}$ & 0.72678000 & 8.15866400 & 5.92278900 \\
\hline $\mathrm{C}$ & 0.93462700 & 3.27256300 & 2.62926500 \\
\hline $\mathrm{C}$ & 1.32237500 & 1.97889500 & 5.10100800 \\
\hline $\mathrm{C}$ & 2.18699500 & 3.29112500 & 3.26144200 \\
\hline $\mathrm{C}$ & -0.11902800 & 2.59409000 & 3.25819200 \\
\hline $\mathrm{C}$ & 0.07138200 & 1.95213200 & 4.48299800 \\
\hline $\mathrm{C}$ & 2.38045500 & 2.65013100 & 4.48519500 \\
\hline $\mathrm{H}$ & 3.01242400 & 3.81618000 & 2.78861100 \\
\hline $\mathrm{H}$ & -1.09778300 & 2.57663700 & 2.78652100 \\
\hline $\mathrm{H}$ & -0.75818200 & 1.42808800 & 4.94983600 \\
\hline $\mathrm{H}$ & 3.35822900 & 2.67844300 & 4.95927800 \\
\hline $\mathrm{H}$ & 1.47141400 & 1.48415800 & 6.05737700 \\
\hline $\mathrm{C}$ & -0.23659100 & -6.01274200 & -2.45550400 \\
\hline $\mathrm{C}$ & 0.14483500 & -7.27345600 & -4.94506800 \\
\hline $\mathrm{C}$ & 0.97843800 & -6.64324800 & -2.76295200 \\
\hline $\mathrm{C}$ & -1.25579000 & -6.02446500 & -3.41924000 \\
\hline $\mathrm{C}$ & -1.06953600 & -6.65162600 & -4.65126300 \\
\hline $\mathrm{C}$ & 1.16878400 & -7.26574600 & -3.99640800 \\
\hline $\mathrm{H}$ & 1.77712000 & -6.64815100 & -2.02683100 \\
\hline $\mathrm{H}$ & -2.20344700 & -5.54174000 & -3.19706200 \\
\hline
\end{tabular}




\begin{tabular}{|c|c|c|c|}
\hline $\mathrm{H}$ & -1.87420800 & -6.65292200 & -5.38205000 \\
\hline $\mathrm{H}$ & 2.11879500 & -7.74638500 & -4.21543600 \\
\hline $\mathrm{H}$ & 0.29216300 & -7.76033700 & -5.90547800 \\
\hline $\mathrm{C}$ & -0.27900400 & -7.54797600 & 0.04564900 \\
\hline $\mathrm{C}$ & 0.00911000 & -10.34912300 & -0.01163200 \\
\hline $\mathrm{C}$ & -1.26689200 & -8.36059100 & -0.52985500 \\
\hline $\mathrm{C}$ & 0.85622200 & -8.16489000 & 0.59164700 \\
\hline $\mathrm{C}$ & 1.00111000 & -9.55173000 & 0.56139900 \\
\hline $\mathrm{C}$ & -1.12671700 & -9.74797100 & -0.55620000 \\
\hline $\mathrm{H}$ & -2.15007300 & -7.89899300 & -0.96223200 \\
\hline $\mathrm{H}$ & 1.62999800 & -7.55142400 & 1.04414900 \\
\hline $\mathrm{H}$ & 1.89020500 & -10.00916200 & 0.98785800 \\
\hline $\mathrm{H}$ & -1.90570200 & -10.35908800 & -1.00486900 \\
\hline $\mathrm{H}$ & 0.12041400 & -11.43003000 & -0.03363900 \\
\hline $\mathrm{C}$ & -0.61796000 & -6.14434300 & 2.58156400 \\
\hline $\mathrm{C}$ & -0.69666400 & -7.59766100 & 4.99240700 \\
\hline $\mathrm{C}$ & -1.70867500 & -6.97128500 & 2.88666600 \\
\hline $\mathrm{C}$ & 0.43307200 & -6.05951200 & 3.50589700 \\
\hline $\mathrm{C}$ & 0.39641400 & -6.78100400 & 4.69917700 \\
\hline $\mathrm{C}$ & -1.75017800 & -7.68953100 & 4.08151000 \\
\hline $\mathrm{H}$ & -2.52824700 & -7.05319300 & 2.17828000 \\
\hline $\mathrm{H}$ & 1.28588800 & -5.42359500 & 3.28604800 \\
\hline $\mathrm{H}$ & 1.22315300 & -6.70332000 & 5.40070600 \\
\hline $\mathrm{H}$ & -2.60603100 & -8.32337700 & 4.29907300 \\
\hline $\mathrm{H}$ & -0.72678000 & -8.15866400 & 5.92278900 \\
\hline $\mathrm{C}$ & -0.93462700 & -3.27256300 & 2.62926500 \\
\hline $\mathrm{C}$ & -1.32237500 & -1.97889500 & 5.10100800 \\
\hline $\mathrm{C}$ & -2.18699500 & -3.29112500 & 3.26144200 \\
\hline $\mathrm{C}$ & 0.11902800 & -2.59409000 & 3.25819200 \\
\hline $\mathrm{C}$ & -0.07138200 & -1.95213200 & 4.48299800 \\
\hline $\mathrm{C}$ & -2.38045500 & -2.65013100 & 4.48519500 \\
\hline $\mathrm{H}$ & -3.01242400 & -3.81618000 & 2.78861100 \\
\hline $\mathrm{H}$ & 1.09778300 & -2.57663700 & 2.78652100 \\
\hline $\mathrm{H}$ & 0.75818200 & -1.42808800 & 4.94983600 \\
\hline $\mathrm{H}$ & -3.35822900 & -2.67844300 & 4.95927800 \\
\hline $\mathrm{H}$ & -1.47141400 & -1.48415800 & 6.05737700 \\
\hline $\mathrm{H}$ & 1.65436600 & 1.42402600 & -0.66991000 \\
\hline $\mathrm{H}$ & 1.59657600 & 1.50339400 & 1.07395200 \\
\hline $\mathrm{C}$ & -0.20808200 & 0.74662700 & 0.17035000 \\
\hline $\mathrm{H}$ & -0.83520700 & 0.94795900 & -0.70543000 \\
\hline $\mathrm{H}$ & -1.59657600 & -1.50339400 & 1.07395200 \\
\hline $\mathrm{C}$ & 0.20808200 & -0.74662700 & 0.17035000 \\
\hline $\mathrm{H}$ & 0.83520700 & -0.94795900 & -0.70543000 \\
\hline $\mathrm{H}$ & 0.82794500 & -0.94797000 & 1.05345900 \\
\hline $\mathrm{H}$ & -1.65436600 & -1.42402600 & -0.66991000 \\
\hline $\mathrm{H}$ & -0.82794500 & 0.94797000 & 1.05345900 \\
\hline
\end{tabular}


Compound 7d: $\quad \mathrm{E}(\mathrm{RB} 3 \mathrm{LYP})=--3121.59761365$ a.u.

$\begin{array}{lrrr}\mathrm{C} & -2.77482400 & 1.88778100 & 1.41516700 \\ \mathrm{C} & -2.43983800 & -0.58793200 & 2.70668600 \\ \mathrm{C} & -3.01365300 & 0.67070200 & 0.71162700 \\ \mathrm{C} & -2.24912900 & 1.81643200 & 2.71412700 \\ \mathrm{C} & -1.94329400 & 0.58195800 & 3.29738800 \\ \mathrm{C} & -2.97541600 & -0.57080300 & 1.41319300 \\ \mathrm{H} & -1.93285400 & 2.73450700 & 3.20444700 \\ \mathrm{H} & -2.26425300 & -1.54406700 & 3.19264300 \\ \mathrm{C} & -3.01365300 & 0.67070200 & -0.71162700 \\ \mathrm{C} & -1.94329400 & 0.58195800 & -3.29738800 \\ \mathrm{C} & -2.77482400 & 1.88778100 & -1.41516700 \\ \mathrm{C} & -2.97541600 & -0.57080300 & -1.41319300 \\ \mathrm{C} & -2.43983800 & -0.58793200 & -2.70668600 \\ \mathrm{C} & -2.24912900 & 1.81643200 & -2.71412700 \\ \mathrm{H} & -2.26425300 & -1.54406700 & -3.19264300 \\ \mathrm{H} & -1.93285400 & 2.73450700 & -3.20444700 \\ \mathrm{C} & -2.84228300 & 3.12584100 & 0.68105100 \\ \mathrm{H} & -2.79094100 & 4.06187400 & 1.23222400 \\ \mathrm{C} & -2.84228300 & 3.12584100 & -0.68105100 \\ \mathrm{H} & -2.79094100 & 4.06187400 & -1.23222400 \\ \mathrm{C} & -3.23959900 & -1.78334300 & 0.68029500 \\ \mathrm{H} & -3.33831900 & -2.71391000 & 1.23394800 \\ \mathrm{C} & -3.23959900 & -1.78334300 & -0.68029500 \\ \mathrm{H} & -3.33831900 & -2.71391000 & -1.23394800 \\ \mathrm{C} & -0.87896300 & 0.44570500 & -4.34593100 \\ \mathrm{C} & 1.18548600 & -0.10304200 & -6.17547400 \\ \mathrm{C} & -1.19370600 & 0.04355700 & -5.65706300 \\ \mathrm{C} & 0.47188900 & 0.59574400 & -3.92859000 \\ \mathrm{C} & 1.49523500 & 0.29345200 & -4.85319000 \\ \mathrm{C} & -0.15979800 & -0.21701200 & -6.58503700 \\ \mathrm{C} & -0.87896300 & 0.44570500 & 4.34593100 \\ \mathrm{C} & 1.18548600 & -0.10304200 & 6.17547400 \\ \mathrm{C} & 0.47188900 & 0.59574400 & 3.92859000 \\ \mathrm{C} & -1.19370600 & 0.04355700 & 5.65706300 \\ \mathrm{C} & -0.15979800 & -0.21701200 & 6.58503700 \\ \mathrm{C} & 1.49523500 & 0.29345200 & 4.85319000 \\ \mathrm{C} & -2.63243800 & -0.12308100 & -6.04265200 \\ \mathrm{C} & -5.34634200 & -0.43857000 & -6.72730200 \\ \mathrm{C} & -3.50365400 & 0.97637000 & -6.05465400 \\ \mathrm{C} & -3.14660000 & -1.38395000 & -6.38024100 \\ \mathrm{C} & -4.49086100 & -1.54158900 & -6.71639700 \\ \mathrm{C} & -4.84714700 & 0.82207400 & -6.39670900 \\ \mathrm{H} & -3.12184300 & 1.96063900 & -5.79805200 \\ \mathrm{H} & -2.48536200 & -2.24561100 & -6.38122900 \\ & & & \end{array}$




\begin{tabular}{|c|c|c|c|}
\hline $\mathrm{H}$ & -4.86939000 & -2.52795200 & -6.97187400 \\
\hline $\mathrm{H}$ & -5.50396000 & 1.68817300 & -6.40356800 \\
\hline $\mathrm{H}$ & -6.39339100 & -0.56045800 & -6.99178900 \\
\hline $\mathrm{C}$ & -0.49565400 & -0.60868700 & -7.99295900 \\
\hline $\mathrm{C}$ & -1.13777600 & -1.33327500 & -10.63788700 \\
\hline $\mathrm{C}$ & -1.14366300 & 0.29435400 & -8.84878700 \\
\hline $\mathrm{C}$ & -0.17634300 & -1.88217300 & -8.48670400 \\
\hline $\mathrm{C}$ & -0.49613100 & -2.24264500 & -9.79556300 \\
\hline $\mathrm{C}$ & -1.45939500 & -0.06214500 & -10.15971200 \\
\hline $\mathrm{H}$ & -1.40354300 & 1.28281600 & -8.48056500 \\
\hline $\mathrm{H}$ & 0.32932900 & -2.59318700 & -7.83975700 \\
\hline $\mathrm{H}$ & -0.24173300 & -3.23557100 & -10.15747300 \\
\hline $\mathrm{H}$ & -1.95911900 & 0.65422200 & -10.80675600 \\
\hline $\mathrm{H}$ & -1.38536200 & -1.61316800 & -11.65848400 \\
\hline $\mathrm{C}$ & 2.29249500 & -0.36657600 & -7.15418100 \\
\hline $\mathrm{C}$ & 4.36827000 & -0.84701700 & -8.99549100 \\
\hline $\mathrm{C}$ & 2.51315200 & 0.50400300 & -8.23135300 \\
\hline $\mathrm{C}$ & 3.13095800 & -1.48199300 & -7.01595200 \\
\hline $\mathrm{C}$ & 4.15766000 & -1.72255900 & -7.92915600 \\
\hline $\mathrm{C}$ & 3.54229500 & 0.26837100 & -9.14270200 \\
\hline $\mathrm{H}$ & 1.86855800 & 1.36942800 & -8.35628500 \\
\hline $\mathrm{H}$ & 2.97591600 & -2.16481600 & -6.18552300 \\
\hline $\mathrm{H}$ & 4.79430500 & -2.59493300 & -7.80551500 \\
\hline $\mathrm{H}$ & 3.69694500 & 0.95655400 & -9.96977900 \\
\hline $\mathrm{H}$ & 5.16929300 & -1.03286100 & -9.70617400 \\
\hline $\mathrm{C}$ & 2.93456200 & 0.41912500 & -4.44216600 \\
\hline $\mathrm{C}$ & 5.63031000 & 0.65643800 & -3.66003200 \\
\hline $\mathrm{C}$ & 3.53137100 & -0.54540200 & -3.61670800 \\
\hline $\mathrm{C}$ & 3.71348300 & 1.50444100 & -4.86944500 \\
\hline $\mathrm{C}$ & 5.04804800 & 1.62367900 & -4.48123000 \\
\hline $\mathrm{C}$ & 4.86736600 & -0.42981300 & -3.22965400 \\
\hline $\mathrm{H}$ & 2.94357100 & -1.39672200 & -3.28363200 \\
\hline $\mathrm{H}$ & 3.26749600 & 2.25808900 & -5.51236400 \\
\hline $\mathrm{H}$ & 5.63401900 & 2.47298600 & -4.82299100 \\
\hline $\mathrm{H}$ & 5.31153200 & -1.19060400 & -2.59276100 \\
\hline $\mathrm{H}$ & 6.67058900 & 0.74815600 & -3.35923000 \\
\hline $\mathrm{C}$ & -2.63243800 & -0.12308100 & 6.04265200 \\
\hline $\mathrm{C}$ & -5.34634200 & -0.43857000 & 6.72730200 \\
\hline $\mathrm{C}$ & -3.14660000 & -1.38395000 & 6.38024100 \\
\hline $\mathrm{C}$ & -3.50365400 & 0.97637000 & 6.05465400 \\
\hline $\mathrm{C}$ & -4.84714700 & 0.82207400 & 6.39670900 \\
\hline $\mathrm{C}$ & -4.49086100 & -1.54158900 & 6.71639700 \\
\hline $\mathrm{H}$ & -2.48536200 & -2.24561100 & 6.38122900 \\
\hline $\mathrm{H}$ & -3.12184300 & 1.96063900 & 5.79805200 \\
\hline $\mathrm{H}$ & -5.50396000 & 1.68817300 & 6.40356800 \\
\hline $\mathrm{H}$ & -4.86939000 & -2.52795200 & 6.97187400 \\
\hline
\end{tabular}




\begin{tabular}{|c|c|c|c|}
\hline $\mathrm{H}$ & -6.39339100 & -0.56045800 & 6.99178900 \\
\hline $\mathrm{C}$ & -0.49565400 & -0.60868700 & 7.99295900 \\
\hline $\mathrm{C}$ & -1.13777600 & -1.33327500 & 10.63788700 \\
\hline $\mathrm{C}$ & -1.14366300 & 0.29435400 & 8.84878700 \\
\hline $\mathrm{C}$ & -0.17634300 & -1.88217300 & 8.48670400 \\
\hline $\mathrm{C}$ & -0.49613100 & -2.24264500 & 9.79556300 \\
\hline $\mathrm{C}$ & -1.45939500 & -0.06214500 & 10.15971200 \\
\hline $\mathrm{H}$ & -1.40354300 & 1.28281600 & 8.48056500 \\
\hline $\mathrm{H}$ & 0.32932900 & -2.59318700 & 7.83975700 \\
\hline $\mathrm{H}$ & -0.24173300 & -3.23557100 & 10.15747300 \\
\hline $\mathrm{H}$ & -1.95911900 & 0.65422200 & 10.80675600 \\
\hline $\mathrm{H}$ & -1.38536200 & -1.61316800 & 11.65848400 \\
\hline $\mathrm{C}$ & 2.29249500 & -0.36657600 & 7.15418100 \\
\hline $\mathrm{C}$ & 4.36827000 & -0.84701700 & 8.99549100 \\
\hline $\mathrm{C}$ & 2.51315200 & 0.50400300 & 8.23135300 \\
\hline $\mathrm{C}$ & 3.13095800 & -1.48199300 & 7.01595200 \\
\hline $\mathrm{C}$ & 4.15766000 & -1.72255900 & 7.92915600 \\
\hline $\mathrm{C}$ & 3.54229500 & 0.26837100 & 9.14270200 \\
\hline $\mathrm{H}$ & 1.86855800 & 1.36942800 & 8.35628500 \\
\hline $\mathrm{H}$ & 2.97591600 & -2.16481600 & 6.18552300 \\
\hline $\mathrm{H}$ & 4.79430500 & -2.59493300 & 7.80551500 \\
\hline $\mathrm{H}$ & 3.69694500 & 0.95655400 & 9.96977900 \\
\hline $\mathrm{H}$ & 5.16929300 & -1.03286100 & 9.70617400 \\
\hline $\mathrm{C}$ & 2.93456200 & 0.41912500 & 4.44216600 \\
\hline $\mathrm{C}$ & 5.63031000 & 0.65643800 & 3.66003200 \\
\hline $\mathrm{C}$ & 3.71348300 & 1.50444100 & 4.86944500 \\
\hline $\mathrm{C}$ & 3.53137100 & -0.54540200 & 3.61670800 \\
\hline $\mathrm{C}$ & 4.86736600 & -0.42981300 & 3.22965400 \\
\hline $\mathrm{C}$ & 5.04804800 & 1.62367900 & 4.48123000 \\
\hline $\mathrm{H}$ & 3.26749600 & 2.25808900 & 5.51236400 \\
\hline $\mathrm{H}$ & 2.94357100 & -1.39672200 & 3.28363200 \\
\hline $\mathrm{H}$ & 5.31153200 & -1.19060400 & 2.59276100 \\
\hline $\mathrm{H}$ & 5.63401900 & 2.47298600 & 4.82299100 \\
\hline $\mathrm{H}$ & 6.67058900 & 0.74815600 & 3.35923000 \\
\hline $\mathrm{C}$ & 0.83684800 & 1.15672000 & 2.54721500 \\
\hline $\mathrm{H}$ & 1.84421500 & 1.57819400 & 2.60935900 \\
\hline $\mathrm{C}$ & 0.78809400 & 0.21888700 & 1.30990500 \\
\hline $\mathrm{H}$ & -0.17391600 & -0.30177300 & 1.27362500 \\
\hline $\mathrm{C}$ & 0.83684800 & 1.15672000 & -2.54721500 \\
\hline $\mathrm{H}$ & 1.84421500 & 1.57819400 & -2.60935900 \\
\hline $\mathrm{C}$ & 0.78809400 & 0.21888700 & -1.30990500 \\
\hline $\mathrm{H}$ & -0.17391600 & -0.30177300 & -1.27362500 \\
\hline $\mathrm{C}$ & 0.98816200 & 1.01361500 & 0.00000000 \\
\hline $\mathrm{H}$ & 1.99008700 & 1.46807100 & 0.00000000 \\
\hline $\mathrm{H}$ & 0.27668200 & 1.85190500 & 0.00000000 \\
\hline $\mathrm{H}$ & 0.17361500 & 2.00066900 & -2.33822600 \\
\hline
\end{tabular}




$\begin{array}{lrrr}\mathrm{H} & 1.56064400 & -0.55568500 & 1.40348200 \\ \mathrm{H} & 1.56064400 & -0.55568500 & -1.40348200 \\ \mathrm{H} & 0.17361500 & 2.00066900 & 2.33822600\end{array}$

Compound 7e: $\quad \mathrm{E}(\mathrm{RB} 3 \mathrm{LYP})=-3160.92635404$ a.u.

$\begin{array}{lrrr}\mathrm{C} & -1.12565600 & -1.50765600 & -2.30882900 \\ \mathrm{C} & 1.40759100 & -2.66838400 & -1.91526800 \\ \mathrm{C} & 0.05238100 & -0.70944700 & -2.41396000 \\ \mathrm{C} & -0.99803400 & -2.84277400 & -1.89948700 \\ \mathrm{C} & 0.25385000 & -3.39050800 & -1.58865300 \\ \mathrm{C} & 1.33501200 & -1.32572700 & -2.31558200 \\ \mathrm{H} & -1.89556900 & -3.42287200 & -1.70142300 \\ \mathrm{H} & 2.38446200 & -3.11167700 & -1.73549200 \\ \mathrm{C} & -0.05238100 & 0.70944700 & -2.41396000 \\ \mathrm{C} & -0.25385000 & 3.39050800 & -1.58865300 \\ \mathrm{C} & -1.33501200 & 1.32572700 & -2.31558200 \\ \mathrm{C} & 1.12565600 & 1.50765600 & -2.30882900 \\ \mathrm{C} & 0.99803400 & 2.84277400 & -1.89948700 \\ \mathrm{C} & -1.40759100 & 2.66838400 & -1.91526800 \\ \mathrm{H} & 1.89556900 & 3.42287200 & -1.70142300 \\ \mathrm{H} & -2.38446200 & 3.11167700 & -1.73549200 \\ \mathrm{C} & -2.40557300 & -0.86117200 & -2.43686600 \\ \mathrm{H} & -3.29979600 & -1.47943000 & -2.45222700 \\ \mathrm{C} & -2.50577800 & 0.49770100 & -2.44309300 \\ \mathrm{H} & -3.48155100 & 0.97729700 & -2.46320600 \\ \mathrm{C} & 2.50577800 & -0.49770100 & -2.44309300 \\ \mathrm{H} & 3.48155100 & -0.97729700 & -2.46320600 \\ \mathrm{C} & 2.40557300 & 0.86117200 & -2.43686600 \\ \mathrm{H} & 3.29979600 & 1.47943000 & -2.45222700 \\ \mathrm{C} & -0.31917300 & 4.60796400 & -0.71323200 \\ \mathrm{C} & -0.22311100 & 6.81609600 & 1.02766000 \\ \mathrm{C} & -0.00191200 & 5.88454900 & -1.21689600 \\ \mathrm{C} & -0.59804400 & 4.41535400 & 0.66570800 \\ \mathrm{C} & -0.52063200 & 5.52722600 & 1.53040700 \\ \mathrm{C} & 0.03090100 & 6.99926500 & -0.34827000 \\ \mathrm{C} & 0.31917300 & -4.60796400 & -0.71323200 \\ \mathrm{C} & 0.22311100 & -6.81609600 & 1.02766000 \\ \mathrm{C} & 0.59804400 & -4.41535400 & 0.66570800 \\ \mathrm{C} & 0.00191200 & -5.88454900 & -1.21689600 \\ \mathrm{C} & -0.03090100 & -6.99926500 & -0.34827000 \\ \mathrm{C} & 0.52063200 & -5.52722600 & 1.53040700 \\ \mathrm{C} & 0.31917300 & 6.04011800 & -2.67301000 \\ \mathrm{C} & 0.93287200 & 6.30643800 & -5.40913900 \\ \mathrm{C} & -0.64984000 & 5.78758700 & -3.65522000 \\ & & & \\ & & & \end{array}$




$\begin{array}{lrcc}\mathrm{C} & 1.60220300 & 6.42765300 & -3.08764600 \\ \mathrm{C} & 1.90778800 & 6.55690900 & -4.44216700 \\ \mathrm{C} & -0.34823100 & 5.92283600 & -5.01056700 \\ \mathrm{H} & -1.64904300 & 5.48702400 & -3.35270700 \\ \mathrm{H} & 2.36347600 & 6.63267000 & -2.34070800 \\ \mathrm{H} & 2.90899800 & 6.85575800 & -4.74173300 \\ \mathrm{H} & -1.11549500 & 5.72656500 & -5.75510100 \\ \mathrm{H} & 1.16972500 & 6.40940300 & -6.46482900 \\ \mathrm{C} & 0.32880900 & 8.36498700 & -0.88992500 \\ \mathrm{C} & 0.88353600 & 10.92720300 & -1.92005000 \\ \mathrm{C} & -0.54733400 & 8.98105000 & -1.79549500 \\ \mathrm{C} & 1.48939600 & 9.05625200 & -0.51144300 \\ \mathrm{C} & 1.76610700 & 10.32398400 & -1.02248700 \\ \mathrm{C} & -0.27543800 & 10.25086400 & -2.30374900 \\ \mathrm{H} & -1.44747900 & 8.45757300 & -2.10489900 \\ \mathrm{H} & 2.17793100 & 8.59554000 & 0.19079400 \\ \mathrm{H} & 2.67231400 & 10.84094800 & -0.71740100 \\ \mathrm{H} & -0.96984900 & 10.71081900 & -3.00217200 \\ \mathrm{H} & 1.09752500 & 11.91592300 & -2.31745500 \\ \mathrm{C} & -0.22139900 & 7.99531400 & 1.95565900 \\ \mathrm{C} & -0.24945200 & 10.21070700 & 3.69452400 \\ \mathrm{C} & -1.22442800 & 8.97157100 & 1.86046000 \\ \mathrm{C} & 0.76563200 & 8.14810900 & 2.93984200 \\ \mathrm{C} & 0.75455800 & 9.24701600 & 3.79940800 \\ \mathrm{C} & -1.60220300 & -6.42765300 & -3.08764600 \\ \mathrm{C} & -1.90778800 & -6.55690900 & -4.44216700 \\ \mathrm{H} & -1.24025300 & 10.06811000 & 2.72199200 \\ \mathrm{H} & -1.99499100 & 8.87016500 & 1.10150800 \\ \mathrm{H} & 1.54741900 & 7.39958100 & 3.03175700 \\ \mathrm{H} & 1.53149500 & 9.34814200 & 4.55288500 \\ \mathrm{H} & -2.02722100 & 10.81253500 & 2.63164800 \\ \mathrm{H} & -0.25973800 & 11.06571800 & 4.36543800 \\ \mathrm{C} & -0.78539200 & 5.35007900 & 2.99763200 \\ \mathrm{C} & -1.27808000 & 4.99829500 & 5.74813700 \\ \mathrm{C} & 0.17504500 & 4.75636100 & 3.82996700 \\ \mathrm{C} & -1.99618100 & 5.76789800 & 3.56918900 \\ \mathrm{C} & -2.24177600 & 5.59177400 & 4.93087600 \\ \mathrm{H} & -0.06721900 & 4.58233700 & 5.19336500 \\ \mathrm{H} & 1.12156000 & 4.43534700 & 3.40326300 \\ \mathrm{H} & -2.74832400 & 6.23498600 & 2.93965400 \\ \mathrm{H} & -3.18735500 & 5.92071800 & 5.35412600 \\ \mathrm{H} & -0.319170800 & 4.12261000 & 5.82135400 \\ \mathrm{H} & 0.6498900 & 4.86334600 & 6.80953700 \\ \mathrm{H} & -6.04011800 & -2.67301000 \\ \mathrm{H} & -6.30643800 & -5.40913900 \\ \mathrm{H} & & & \\ \mathrm{H} & & & \end{array}$




\begin{tabular}{|c|c|c|c|}
\hline $\mathrm{C}$ & 0.34823100 & -5.92283600 & -5.01056700 \\
\hline $\mathrm{H}$ & 1.64904300 & -5.48702400 & -3.35270700 \\
\hline $\mathrm{H}$ & -2.36347600 & -6.63267000 & -2.34070800 \\
\hline $\mathrm{H}$ & -2.90899800 & -6.85575800 & -4.74173300 \\
\hline $\mathrm{H}$ & 1.11549500 & -5.72656500 & -5.75510100 \\
\hline $\mathrm{H}$ & -1.16972500 & -6.40940300 & -6.46482900 \\
\hline $\mathrm{C}$ & -0.32880900 & -8.36498700 & -0.88992500 \\
\hline $\mathrm{C}$ & -0.88353600 & -10.92720300 & -1.92005000 \\
\hline $\mathrm{C}$ & -1.48939600 & -9.05625200 & -0.51144300 \\
\hline $\mathrm{C}$ & 0.54733400 & -8.98105000 & -1.79549500 \\
\hline $\mathrm{C}$ & 0.27543800 & -10.25086400 & -2.30374900 \\
\hline $\mathrm{C}$ & -1.76610700 & -10.32398400 & -1.02248700 \\
\hline $\mathrm{H}$ & -2.17793100 & -8.59554000 & 0.19079400 \\
\hline $\mathrm{H}$ & 1.44747900 & -8.45757300 & -2.10489900 \\
\hline $\mathrm{H}$ & 0.96984900 & -10.71081900 & -3.00217200 \\
\hline $\mathrm{H}$ & -2.67231400 & -10.84094800 & -0.71740100 \\
\hline $\mathrm{H}$ & -1.09752500 & -11.91592300 & -2.31745500 \\
\hline $\mathrm{C}$ & 0.22139900 & -7.99531400 & 1.95565900 \\
\hline $\mathrm{C}$ & 0.24945200 & -10.21070700 & 3.69452400 \\
\hline $\mathrm{C}$ & -0.76563200 & -8.14810900 & 2.93984200 \\
\hline $\mathrm{C}$ & 1.22442800 & -8.97157100 & 1.86046000 \\
\hline $\mathrm{C}$ & 1.24025300 & -10.06811000 & 2.72199200 \\
\hline $\mathrm{C}$ & -0.75455800 & -9.24701600 & 3.79940800 \\
\hline $\mathrm{H}$ & -1.54741900 & -7.39958100 & 3.03175700 \\
\hline $\mathrm{H}$ & 1.99499100 & -8.87016500 & 1.10150800 \\
\hline $\mathrm{H}$ & 2.02722100 & -10.81253500 & 2.63164800 \\
\hline $\mathrm{H}$ & -1.53149500 & -9.34814200 & 4.55288500 \\
\hline $\mathrm{H}$ & 0.25973800 & -11.06571800 & 4.36543800 \\
\hline $\mathrm{C}$ & 0.78539200 & -5.35007900 & 2.99763200 \\
\hline $\mathrm{C}$ & 1.27808000 & -4.99829500 & 5.74813700 \\
\hline $\mathrm{C}$ & -0.17504500 & -4.75636100 & 3.82996700 \\
\hline $\mathrm{C}$ & 1.99618100 & -5.76789800 & 3.56918900 \\
\hline $\mathrm{C}$ & 2.24177600 & -5.59177400 & 4.93087600 \\
\hline $\mathrm{C}$ & 0.06721900 & -4.58233700 & 5.19336500 \\
\hline $\mathrm{H}$ & -1.12156000 & -4.43534700 & 3.40326300 \\
\hline $\mathrm{H}$ & 2.74832400 & -6.23498600 & 2.93965400 \\
\hline $\mathrm{H}$ & 3.18735500 & -5.92071800 & 5.35412600 \\
\hline $\mathrm{H}$ & -0.69170800 & -4.12261000 & 5.82135400 \\
\hline $\mathrm{H}$ & 1.46879900 & -4.86334600 & 6.80953700 \\
\hline $\mathrm{C}$ & -0.05706300 & -1.97926600 & 1.42725300 \\
\hline $\mathrm{H}$ & -0.65222500 & -2.24045300 & 2.31225900 \\
\hline $\mathrm{C}$ & 0.52936700 & -0.55972800 & 1.58159600 \\
\hline $\mathrm{H}$ & 1.21958600 & -0.38040000 & 0.74540400 \\
\hline $\mathrm{C}$ & -1.04037100 & 3.05172700 & 1.20610500 \\
\hline $\mathrm{H}$ & -1.57256100 & 3.20515900 & 2.14892700 \\
\hline $\mathrm{C}$ & 0.05706300 & 1.97926600 & 1.42725300 \\
\hline
\end{tabular}




$\begin{array}{lrrr}\mathrm{H} & 0.74785500 & 1.98116800 & 0.57805300 \\ \mathrm{C} & -0.52936700 & 0.55972800 & 1.58159600 \\ \mathrm{H} & -1.13735500 & 0.50412200 & 2.49648700 \\ \mathrm{H} & -1.21958600 & 0.38040000 & 0.74540400 \\ \mathrm{H} & -1.77570600 & 2.63186200 & 0.51378300 \\ \mathrm{H} & 1.13735500 & -0.50412200 & 2.49648700 \\ \mathrm{H} & 0.65222500 & 2.24045300 & 2.31225900 \\ \mathrm{H} & -0.74785500 & -1.98116800 & 0.57805300 \\ \mathrm{C} & 1.04037100 & -3.05172700 & 1.20610500 \\ \mathrm{H} & 1.57256100 & -3.20515900 & 2.14892700 \\ \mathrm{H} & 1.77570600 & -2.63186200 & 0.51378300\end{array}$

Compound 13a: $\quad E(R B 3 L Y P)=-3803.64284607$ a.u

$\begin{array}{lrrr}\mathrm{C} & -1.69339800 & 4.28725500 & 1.12028100 \\ \mathrm{C} & -2.05234400 & 2.83854000 & -1.25286900 \\ \mathrm{C} & -1.55627500 & 4.90820400 & -0.12365000 \\ \mathrm{C} & -2.04720000 & 2.93686700 & 1.15542900 \\ \mathrm{C} & -2.24497900 & 2.20129200 & -0.02162700 \\ \mathrm{C} & -1.70127500 & 4.19172100 & -1.31530000 \\ \mathrm{H} & -2.16566500 & 2.44096100 & 2.11544200 \\ \mathrm{H} & -2.17233800 & 2.27377000 & -2.17298000 \\ \mathrm{C} & 1.55628600 & 4.90820300 & 0.12365300 \\ \mathrm{C} & 2.24498500 & 2.20129000 & 0.02163200 \\ \mathrm{C} & 1.70128500 & 4.19172000 & 1.31530500 \\ \mathrm{C} & 1.69340800 & 4.28725300 & -1.12027700 \\ \mathrm{C} & 2.04720700 & 2.93686400 & -1.15542400 \\ \mathrm{C} & 2.05235200 & 2.83853900 & 1.25287400 \\ \mathrm{H} & 2.16567100 & 2.44095700 & -2.11543600 \\ \mathrm{H} & 2.17234500 & 2.27376900 & 2.17298500 \\ \mathrm{C} & 2.70605100 & 0.77883700 & -0.10900200 \\ \mathrm{C} & 3.73346200 & -1.75802200 & -0.76667800 \\ \mathrm{C} & 4.05418400 & 0.47088900 & 0.16822900 \\ \mathrm{C} & 1.83767900 & -0.20544100 & -0.63516700 \\ \mathrm{C} & 2.37591400 & -1.45455700 & -1.02307400 \\ \mathrm{C} & 4.56146000 & -0.81068400 & -0.13025400 \\ \mathrm{C} & -2.70604900 & 0.77884000 & 0.10900800 \\ \mathrm{C} & -3.73346600 & -1.75801700 & 0.76667900 \\ \mathrm{C} & -1.83768000 & -0.20543900 & 0.63517500 \\ \mathrm{C} & -4.05418100 & 0.47089500 & -0.16822800 \\ \mathrm{C} & -4.56146000 & -0.81067600 & 0.13025300 \\ \mathrm{C} & -2.37591800 & -1.45455500 & 1.02307900 \\ \mathrm{C} & -0.33549600 & 0.04983300 & 0.70630300 \\ \mathrm{H} & -0.12377900 & 1.00371400 & 1.19825600 \\ \mathrm{H} & 0.13643700 & -0.72730800 & 1.30816100\end{array}$




\begin{tabular}{|c|c|c|c|}
\hline $\mathrm{C}$ & 0.33549600 & 0.04983500 & -0.70629400 \\
\hline $\mathrm{H}$ & 0.12378100 & 1.00371800 & -1.19824300 \\
\hline $\mathrm{H}$ & -0.13643900 & -0.72730300 & -1.30815400 \\
\hline $\mathrm{C}$ & 4.96942400 & 1.52559000 & 0.72177700 \\
\hline $\mathrm{C}$ & 6.67876700 & 3.51534200 & 1.74388200 \\
\hline $\mathrm{C}$ & 5.16524800 & 1.66296800 & 2.10286900 \\
\hline $\mathrm{C}$ & 5.64693500 & 2.39999800 & -0.13873700 \\
\hline $\mathrm{C}$ & 6.49395600 & 3.38686400 & 0.36656600 \\
\hline $\mathrm{C}$ & 6.01164100 & 2.64904900 & 2.61128500 \\
\hline $\mathrm{H}$ & 4.64844300 & 0.99084000 & 2.78297400 \\
\hline $\mathrm{H}$ & 5.50508100 & 2.30532300 & -1.21186600 \\
\hline $\mathrm{H}$ & 7.01080600 & 4.05511000 & -0.31754700 \\
\hline $\mathrm{H}$ & 6.15114300 & 2.73829500 & 3.68573500 \\
\hline $\mathrm{H}$ & 7.33925100 & 4.28316700 & 2.13819200 \\
\hline $\mathrm{C}$ & 6.00073700 & -1.13118800 & 0.15162500 \\
\hline $\mathrm{C}$ & 8.70023600 & -1.73369500 & 0.67642100 \\
\hline $\mathrm{C}$ & 6.42174700 & -1.47183400 & 1.44453000 \\
\hline $\mathrm{C}$ & 6.95592000 & -1.09616200 & -0.87365300 \\
\hline $\mathrm{C}$ & 8.29408100 & -1.39320800 & -0.61463300 \\
\hline $\mathrm{C}$ & 7.75881000 & -1.77272100 & 1.70582400 \\
\hline $\mathrm{H}$ & 5.69300700 & -1.50576700 & 2.25001400 \\
\hline $\mathrm{H}$ & 6.64487300 & -0.83703100 & -1.88186300 \\
\hline $\mathrm{H}$ & 9.01932000 & -1.35983900 & -1.42363900 \\
\hline $\mathrm{H}$ & 8.06437200 & -2.03738600 & 2.71495200 \\
\hline $\mathrm{H}$ & 9.74228400 & -1.96706400 & 0.87874300 \\
\hline $\mathrm{C}$ & 4.31022700 & -3.07787800 & -1.18884400 \\
\hline $\mathrm{C}$ & 5.40487400 & -5.54662200 & -1.98546200 \\
\hline $\mathrm{C}$ & 4.64181700 & -4.05545200 & -0.24001200 \\
\hline $\mathrm{C}$ & 4.53799100 & -3.35845600 & -2.54380500 \\
\hline $\mathrm{C}$ & 5.08169800 & -4.58035600 & -2.93944500 \\
\hline $\mathrm{C}$ & 5.18161600 & -5.28008000 & -0.63385600 \\
\hline $\mathrm{H}$ & 4.47377000 & -3.85302500 & 0.81398300 \\
\hline $\mathrm{H}$ & 4.28169100 & -2.61356100 & -3.29193000 \\
\hline $\mathrm{H}$ & 5.25133700 & -4.77749500 & -3.99491400 \\
\hline $\mathrm{H}$ & 5.42969400 & -6.02550900 & 0.11746500 \\
\hline $\mathrm{H}$ & 5.82712000 & -6.49973900 & -2.29301600 \\
\hline $\mathrm{C}$ & 1.54119800 & -2.44583700 & -1.78031800 \\
\hline $\mathrm{C}$ & 0.05223200 & -4.30334400 & -3.28907100 \\
\hline $\mathrm{C}$ & 1.10986600 & -2.13942600 & -3.08194200 \\
\hline $\mathrm{C}$ & 1.21035300 & -3.69953900 & -1.24922800 \\
\hline $\mathrm{C}$ & 0.47380100 & -4.61968500 & -1.99679400 \\
\hline $\mathrm{C}$ & 0.37194900 & -3.05673300 & -3.82984900 \\
\hline $\mathrm{H}$ & 1.36329900 & -1.17253600 & -3.50963700 \\
\hline $\mathrm{H}$ & 1.53294300 & -3.95514100 & -0.24562100 \\
\hline $\mathrm{H}$ & 0.23260300 & -5.58927700 & -1.56818100 \\
\hline $\mathrm{H}$ & 0.05217900 & -2.79886000 & -4.83629800 \\
\hline
\end{tabular}




$\begin{array}{lrrr}\mathrm{H} & -0.51807100 & -5.02289600 & -3.87070900 \\ \mathrm{C} & -4.96941700 & 1.52559900 & -0.72177900 \\ \mathrm{C} & -6.67875000 & 3.51535500 & -1.74389100 \\ \mathrm{C} & -5.16523300 & 1.66297800 & -2.10287200 \\ \mathrm{C} & -5.64693000 & 2.40000700 & 0.13873200 \\ \mathrm{C} & -6.49394700 & 3.38687500 & -0.36657400 \\ \mathrm{C} & -6.01162100 & 2.64906100 & -2.61129100 \\ \mathrm{H} & -4.64842500 & 0.99085000 & -2.78297500 \\ \mathrm{H} & -5.50508100 & 2.30533100 & 1.21186200 \\ \mathrm{H} & -7.01079800 & 4.05512200 & 0.31753700 \\ \mathrm{H} & -6.15111800 & 2.73831000 & -3.68574200 \\ \mathrm{H} & -7.33923100 & 4.28318200 & -2.13820400 \\ \mathrm{C} & -6.00073700 & -1.13117800 & -0.15163100 \\ \mathrm{C} & -8.70023500 & -1.73368000 & -0.67643800 \\ \mathrm{C} & -6.95592400 & -1.09615100 & 0.87364300 \\ \mathrm{C} & -6.42174200 & -1.47182300 & -1.44453800 \\ \mathrm{C} & -7.75880500 & -1.77270700 & -1.70583700 \\ \mathrm{C} & -8.29408500 & -1.39319400 & 0.61461700 \\ \mathrm{H} & -6.64488000 & -0.83702000 & 1.88185300 \\ \mathrm{H} & -5.69300000 & -1.50575600 & -2.25002000 \\ \mathrm{H} & -8.06436400 & -2.03737100 & -2.71496700 \\ \mathrm{H} & -9.01932600 & -1.35982400 & 1.42362100 \\ \mathrm{H} & -9.74228300 & -1.96704600 & -0.87876400 \\ \mathrm{C} & -4.31023600 & -3.07787200 & 1.18884300 \\ \mathrm{C} & -5.40489000 & -5.54661400 & 1.98545500 \\ \mathrm{C} & -4.53800400 & -3.35845100 & 2.54380200 \\ \mathrm{C} & -4.64182500 & -4.05544400 & 0.24000900 \\ \mathrm{H} & -5.18162700 & -5.28007100 & 0.63385000 \\ \mathrm{H} & -5.08171500 & -4.58035000 & 2.93944000 \\ \mathrm{C} & -4.28170400 & -2.61355600 & 3.29192900 \\ \mathrm{H} & -4.47377400 & -3.85301700 & -0.81398600 \\ \mathrm{H} & -5.42970500 & -6.02550000 & -0.11747200 \\ \mathrm{H} & -5.25135700 & -4.77748900 & 3.99490800 \\ \mathrm{C} & -1.36331600 & -1.17254100 & 3.50964900 \\ \mathrm{H} & -1.53294800 & -3.95513900 & 0.24562600 \\ \mathrm{C} & -1.10988300 & -2.13943000 & 3.08195200 \\ \mathrm{C} & -1.21036300 & -3.69953900 & 1.24923500 \\ \mathrm{H} & -0.47381700 & -4.61968800 & 1.99680300 \\ \mathrm{H} & -2.58928000 & 1.56819000 \\ \mathrm{H} & -52713900 & -6.49973000 & 2.29300800 \\ \mathrm{H} & -5.02290500 & 3.87072300 \\ \mathrm{H} & -5.96405300 & -0.16658300\end{array}$




$\begin{array}{lrrr}\mathrm{H} & 1.29664100 & 5.96405200 & 0.16658700 \\ \mathrm{C} & -1.44995400 & 5.06030200 & 2.39720000 \\ \mathrm{H} & -2.33487300 & 5.03739700 & 3.04386700 \\ \mathrm{H} & -1.24012000 & 6.11352800 & 2.18197300 \\ \mathrm{C} & 1.44535800 & 4.87325800 & 2.63727400 \\ \mathrm{H} & 1.46202800 & 5.96225800 & 2.52252400 \\ \mathrm{H} & 2.21502200 & 4.61140200 & 3.37156500 \\ \mathrm{~S} & -0.12201000 & 4.40542000 & 3.50839300 \\ \mathrm{C} & -1.44534700 & 4.87325700 & -2.63727000 \\ \mathrm{H} & -1.46201700 & 5.96225800 & -2.52252100 \\ \mathrm{H} & -2.21501200 & 4.61140200 & -3.37156100 \\ \mathrm{C} & 1.44996400 & 5.06030000 & -2.39719700 \\ \mathrm{H} & 2.33488400 & 5.03739300 & -3.04386400 \\ \mathrm{H} & 1.24013200 & 6.11352500 & -2.18197100 \\ \mathrm{~S} & 0.12201900 & 4.40541800 & -3.50838900\end{array}$

Compound 13b: $\quad \mathrm{E}(\mathrm{RB} 3 \mathrm{LYP})=-3842.95657475$ a.u.

$\begin{array}{lrrr}\mathrm{C} & 1.43423700 & -4.43777900 & 1.44559300 \\ \mathrm{C} & 1.85055100 & -2.96800100 & -0.89759500 \\ \mathrm{C} & 1.12581200 & -4.98501300 & 0.19881600 \\ \mathrm{C} & 2.05159100 & -3.18466100 & 1.49064800 \\ \mathrm{C} & 2.25155900 & -2.42141900 & 0.33181900 \\ \mathrm{C} & 1.32122400 & -4.25989700 & -0.98091100 \\ \mathrm{H} & 2.35493000 & -2.78079600 & 2.45274100 \\ \mathrm{H} & 1.97674800 & -2.38749800 & -1.80606500 \\ \mathrm{C} & -1.97810400 & -4.69341800 & 0.43855700 \\ \mathrm{C} & -2.66029000 & -1.98860900 & 0.26861000 \\ \mathrm{C} & -2.08314000 & -3.93961400 & 1.61189900 \\ \mathrm{C} & -2.14501900 & -4.11093600 & -0.81895000 \\ \mathrm{C} & -2.50068500 & -2.76250900 & -0.88712000 \\ \mathrm{C} & -2.42894700 & -2.58490500 & 1.51558400 \\ \mathrm{H} & -2.63576700 & -2.29409900 & -1.85844100 \\ \mathrm{H} & -2.51703100 & -1.98884600 & 2.42045800 \\ \mathrm{C} & -3.06727800 & -0.55148200 & 0.08504700 \\ \mathrm{C} & -3.86660200 & 2.05333400 & -0.62672200 \\ \mathrm{C} & -4.43836600 & -0.23094900 & 0.01656600 \\ \mathrm{C} & -2.08446400 & 0.43310200 & -0.16388900 \\ \mathrm{C} & -2.49200400 & 1.73077900 & -0.54337100 \\ \mathrm{C} & -4.84158300 & 1.07747500 & -0.32723500 \\ \mathrm{C} & 2.90396600 & -1.07041300 & 0.42007800 \\ \mathrm{C} & 4.23326400 & 1.41557900 & 0.61886000 \\ \mathrm{C} & 2.37098800 & -0.05352000 & 1.25268100 \\ \mathrm{C} & 4.09509900 & -0.83572500 & -0.31209500 \\ \mathrm{C} & 4.74135800 & 0.41580100 & -0.23719200\end{array}$




$\begin{array}{lrrr}\mathrm{C} & 3.07363600 & 1.16775900 & 1.37998100 \\ \mathrm{C} & 1.07097800 & -0.23755000 & 2.02938300 \\ \mathrm{H} & 1.22931600 & 0.09682200 & 3.06053500 \\ \mathrm{C} & -0.61956400 & 0.09688900 & 0.07127500 \\ \mathrm{H} & 0.02297200 & 0.59561600 & -0.65837600 \\ \mathrm{C} & -5.46843400 & -1.29463500 & 0.26280400 \\ \mathrm{C} & -7.39953700 & -3.29282700 & 0.71784100 \\ \mathrm{C} & -5.75151000 & -1.73687800 & 1.56271200 \\ \mathrm{C} & -6.16915800 & -1.87197700 & -0.80532300 \\ \mathrm{C} & -7.12454700 & -2.86347100 & -0.58144100 \\ \mathrm{C} & -6.71019600 & -2.72475200 & 1.79010700 \\ \mathrm{H} & -5.22015700 & -1.29610600 & 2.40206400 \\ \mathrm{H} & -5.96388900 & -1.53728600 & -1.81829000 \\ \mathrm{H} & -7.65653500 & -3.29846100 & -1.42369300 \\ \mathrm{H} & -6.91883100 & -3.04972100 & 2.80635000 \\ \mathrm{H} & -8.14625800 & -4.06277800 & 0.89350500 \\ \mathrm{C} & -6.30201200 & 1.40951200 & -0.41363500 \\ \mathrm{C} & -9.04769800 & 2.01453100 & -0.57655800 \\ \mathrm{C} & -7.09639200 & 1.45952800 & 0.74089300 \\ \mathrm{C} & -6.90856000 & 1.66756900 & -1.65149500 \\ \mathrm{C} & -8.26868300 & 1.96508800 & -1.73368800 \\ \mathrm{C} & -8.45588100 & 1.76179100 & 0.66185500 \\ \mathrm{H} & -6.64316700 & 1.25862100 & 1.70751200 \\ \mathrm{H} & -0.81167200 & 1.80153500 & -2.67958800 \\ \mathrm{H} & -6.30682900 & 1.63684300 & -2.55536500 \\ \mathrm{H} & -8.71934600 & 2.15970500 & -2.70355300 \\ \mathrm{H} & -9.05290800 & 1.79780900 & 1.56955500 \\ \mathrm{H} & -10.10727400 & 2.24822300 & -0.63944100 \\ \mathrm{C} & -4.29525100 & 3.43476500 & -1.02704400 \\ \mathrm{C} & -5.09848400 & 6.02849000 & -1.77429400 \\ \mathrm{C} & -4.91450300 & 4.28910700 & -0.10314400 \\ \mathrm{C} & -4.08767000 & 3.90274300 & -2.33263100 \\ \mathrm{H} & -4.48695500 & 5.18648900 & -2.70441100 \\ \mathrm{C} & -1.30240100 & 3.91281300 & -0.06736900 \\ \mathrm{C} & -5.31002400 & 5.57503700 & -0.47132800 \\ \mathrm{H} & -5.08905300 & 3.93976800 & 0.91055900 \\ \mathrm{H} & -3.60700400 & 3.25505100 & -3.06035900 \\ \mathrm{H} & -5.31827100 & 5.52887900 & -3.72223100 \\ \mathrm{H} & -5.40831400 & 7.02954400 & -2.06265000 \\ \mathrm{H} & -1.47150800 & 2.78000200 & -0.87712900 \\ \mathrm{H} & 0.39083300 & 4.78270800 & -1.56373000 \\ \mathrm{H} & -23854700 & 3.65665400 & -2.37525200 \\ \mathrm{H} & -2.02012900 & 0.82887300\end{array}$




$\begin{array}{lrrr}\mathrm{H} & -0.27890300 & 5.78293300 & 0.22626200 \\ \mathrm{H} & 0.83150800 & 3.55138600 & -3.28016300 \\ \mathrm{H} & 1.10055500 & 5.56052000 & -1.83315200 \\ \mathrm{C} & 4.70471600 & -1.92385100 & -1.14821600 \\ \mathrm{C} & 5.86202700 & -3.96798900 & -2.70305000 \\ \mathrm{C} & 4.74734900 & -1.82609700 & -2.54661500 \\ \mathrm{C} & 5.25802500 & -3.06176100 & -0.54289100 \\ \mathrm{C} & 5.83312900 & -4.07367400 & -1.31134800 \\ \mathrm{C} & 5.31634700 & -2.83974500 & -3.31804800 \\ \mathrm{H} & 4.33182400 & -0.94739100 & -3.03144500 \\ \mathrm{H} & 5.23017600 & -3.15423900 & 0.53932100 \\ \mathrm{H} & 6.25860500 & -4.94597900 & -0.82161600 \\ \mathrm{H} & 5.33664000 & -2.74486100 & -4.40073500 \\ \mathrm{H} & 6.30895800 & -4.75640200 & -3.30302400 \\ \mathrm{C} & 5.99720400 & 0.67475100 & -1.01720800 \\ \mathrm{C} & 8.35401800 & 1.17483600 & -2.47516900 \\ \mathrm{C} & 7.20272600 & 0.04798000 & -0.67043500 \\ \mathrm{C} & 5.99440300 & 1.55810600 & -2.10606900 \\ \mathrm{C} & 7.16064900 & 1.80500900 & -2.83068000 \\ \mathrm{C} & 8.37083400 & 0.29591000 & -1.39105900 \\ \mathrm{H} & 7.22211300 & -0.64149700 & 0.16876900 \\ \mathrm{H} & 5.06953300 & 2.05522400 & -2.38492400 \\ \mathrm{H} & 7.13607200 & 2.49195100 & -3.67282300 \\ \mathrm{H} & 9.93518100 & -0.19921600 & -1.10458100 \\ \mathrm{H} & 3.07462300 & 2.74200400 & 5.76379700 \\ \mathrm{H} & & & \end{array}$




$\begin{array}{lrrr}\mathrm{H} & 1.68946800 & 4.73380500 & 5.20293500 \\ \mathrm{H} & 0.70230600 & -5.98572900 & 0.14530200 \\ \mathrm{H} & -1.73233900 & -5.75072200 & 0.50756600 \\ \mathrm{C} & 1.05827200 & -5.15247700 & 2.72170500 \\ \mathrm{H} & 1.91511300 & -5.23798000 & 3.39974600 \\ \mathrm{H} & 0.70231700 & -6.16836600 & 2.52053900 \\ \mathrm{C} & -1.82518700 & -4.60403000 & 2.94403500 \\ \mathrm{H} & -1.94800100 & -5.68906500 & 2.86093400 \\ \mathrm{H} & -2.53882700 & -4.25585200 & 3.69869600 \\ \mathrm{~S} & -0.19696100 & -4.27577400 & 3.77245400 \\ \mathrm{C} & 0.97643700 & -4.88522500 & -2.31253600 \\ \mathrm{H} & 0.90974800 & -5.97421600 & -2.21587400 \\ \mathrm{H} & 1.75958300 & -4.67370100 & -3.04850700 \\ \mathrm{C} & -1.91996500 & -4.90854500 & -2.08154800 \\ \mathrm{H} & -2.79718200 & -4.86206500 & -2.73678300 \\ \mathrm{H} & -1.74288900 & -5.96568000 & -1.85610200 \\ \mathrm{~S} & -0.55451100 & -4.29617500 & -3.17119300 \\ \mathrm{H} & 0.80948800 & -1.29255800 & 2.08869400 \\ \mathrm{H} & -0.46905300 & -0.97657100 & -0.06185100 \\ \mathrm{C} & -0.17871200 & 0.50155300 & 1.49768400 \\ \mathrm{H} & -0.99190000 & 0.26909300 & 2.19765800 \\ \mathrm{H} & -0.04123700 & 1.58619700 & 1.54319200\end{array}$

Compound 13c: $\quad E(R B 3 L Y P)=-3882.26929970$ a.u.

$\begin{array}{lrrr}\mathrm{C} & 1.70665700 & -4.10648300 & 1.45918000 \\ \mathrm{C} & 2.48499100 & -2.71873800 & -0.83851900 \\ \mathrm{C} & 1.58131300 & -4.69086800 & 0.19698500 \\ \mathrm{C} & 2.29996500 & -2.84373900 & 1.55963600 \\ \mathrm{C} & 2.69332000 & -2.13214600 & 0.41963200 \\ \mathrm{C} & 1.94478600 & -4.00199800 & -0.96387300 \\ \mathrm{H} & 2.44684900 & -2.40521000 & 2.54311000 \\ \mathrm{H} & 2.76349900 & -2.16907200 & -1.73278500 \\ \mathrm{C} & -1.52528800 & -4.67772400 & -0.25930800 \\ \mathrm{C} & -2.63764500 & -2.11284600 & -0.41184300 \\ \mathrm{C} & -1.89033100 & -4.02098100 & 0.91956000 \\ \mathrm{C} & -1.64775700 & -4.05849000 & -1.50501200 \\ \mathrm{C} & -2.23795600 & -2.79216300 & -1.56999900 \\ \mathrm{C} & -2.43291100 & -2.73547400 & 0.82903400 \\ \mathrm{H} & -2.38219000 & -2.32356300 & -2.54043700 \\ \mathrm{H} & -2.71713100 & -2.21435600 & 1.73840600 \\ \mathrm{C} & -3.30173200 & -0.76726900 & -0.49837400 \\ \mathrm{C} & -4.62580500 & 1.72377000 & -0.62913600 \\ \mathrm{C} & -4.63863000 & -0.64464100 & -0.04631100 \\ \mathrm{C} & -2.62224300 & 0.35783400 & -1.02600400\end{array}$




\begin{tabular}{|c|c|c|c|}
\hline $\mathrm{C}$ & -3.30233000 & 1.59653100 & -1.09767000 \\
\hline $\mathrm{C}$ & -5.29696700 & 0.60153300 & -0.09999500 \\
\hline $\mathrm{C}$ & 3.34099000 & -0.78065100 & 0.53016300 \\
\hline $\mathrm{C}$ & 4.60808800 & 1.73604200 & 0.66488600 \\
\hline $\mathrm{C}$ & 2.63958300 & 0.32293100 & 1.07579100 \\
\hline $\mathrm{C}$ & 4.66862200 & -0.62723400 & 0.06757700 \\
\hline $\mathrm{C}$ & 5.30187400 & 0.63309200 & 0.12756200 \\
\hline $\mathrm{C}$ & 3.28800000 & 1.57750100 & 1.14052600 \\
\hline $\mathrm{C}$ & 1.22496500 & 0.17756800 & 1.63444100 \\
\hline $\mathrm{H}$ & 0.94575100 & 1.11790000 & 2.11380700 \\
\hline $\mathrm{C}$ & -1.18363300 & 0.28152200 & -1.52683100 \\
\hline $\mathrm{H}$ & -1.10060200 & 0.90243900 & -2.42406700 \\
\hline $\mathrm{C}$ & -5.37842400 & -1.84460400 & 0.47061800 \\
\hline $\mathrm{C}$ & -6.77612200 & -4.09470100 & 1.42881700 \\
\hline $\mathrm{C}$ & -5.73647400 & -1.94566100 & 1.82275600 \\
\hline $\mathrm{C}$ & -5.73851900 & -2.88887100 & -0.39357900 \\
\hline $\mathrm{C}$ & -6.43266200 & -4.00256900 & 0.07898900 \\
\hline $\mathrm{C}$ & -6.42494100 & -3.06147600 & 2.29929100 \\
\hline $\mathrm{H}$ & -5.47363600 & -1.14191100 & 2.50459300 \\
\hline $\mathrm{H}$ & -5.46777400 & -2.82662300 & -1.44391700 \\
\hline $\mathrm{H}$ & -6.70464200 & -4.79926400 & -0.60882200 \\
\hline $\mathrm{H}$ & -6.69081600 & -3.12050000 & 3.35165700 \\
\hline $\mathrm{H}$ & -7.31603600 & -4.96245500 & 1.79866700 \\
\hline $\mathrm{C}$ & -6.71753700 & 0.73308700 & 0.36505200 \\
\hline $\mathrm{C}$ & -9.38392200 & 0.99179300 & 1.23267000 \\
\hline $\mathrm{C}$ & -7.02657300 & 1.44119300 & 1.53514600 \\
\hline $\mathrm{C}$ & -7.76868600 & 0.15840400 & -0.36375700 \\
\hline $\mathrm{C}$ & -9.08991400 & 0.28758700 & 0.06394200 \\
\hline $\mathrm{C}$ & -8.34672700 & 1.56777400 & 1.96761300 \\
\hline $\mathrm{H}$ & -6.22408500 & 1.89557800 & 2.10940500 \\
\hline $\mathrm{H}$ & -7.54657300 & -0.39648800 & -1.27091500 \\
\hline $\mathrm{H}$ & -9.89016900 & -0.16411800 & -0.51675100 \\
\hline $\mathrm{H}$ & -8.56419300 & 2.11911300 & 2.87885600 \\
\hline $\mathrm{H}$ & -10.41312400 & 1.09147900 & 1.56742300 \\
\hline $\mathrm{C}$ & -5.34373700 & 3.03712400 & -0.74936300 \\
\hline $\mathrm{C}$ & -6.69817100 & 5.49397400 & -0.98931300 \\
\hline $\mathrm{C}$ & -5.10814400 & 4.07831300 & 0.15890300 \\
\hline $\mathrm{C}$ & -6.27067300 & 3.24795900 & -1.77957900 \\
\hline $\mathrm{C}$ & -6.94093000 & 4.46538100 & -1.90072100 \\
\hline $\mathrm{C}$ & -5.77968900 & 5.29568700 & 0.04246700 \\
\hline $\mathrm{H}$ & -4.39584900 & 3.93024400 & 0.96616900 \\
\hline $\mathrm{H}$ & -6.46745600 & 2.44968800 & -2.48996500 \\
\hline $\mathrm{H}$ & -7.65457800 & 4.60895200 & -2.70799700 \\
\hline $\mathrm{H}$ & -5.58407000 & 6.08941000 & 0.75902300 \\
\hline $\mathrm{H}$ & -7.22103900 & 6.44225300 & -1.08200500 \\
\hline $\mathrm{C}$ & -2.65120400 & 2.78250500 & -1.75552200 \\
\hline
\end{tabular}




\begin{tabular}{|c|c|c|c|}
\hline $\mathrm{C}$ & -1.47758000 & 5.00070900 & -3.03890000 \\
\hline $\mathrm{C}$ & -2.76300900 & 2.95479200 & -3.14365700 \\
\hline $\mathrm{C}$ & -1.94378000 & 3.74497200 & -1.02197300 \\
\hline $\mathrm{C}$ & -1.36309700 & 4.84433700 & -1.65714300 \\
\hline $\mathrm{C}$ & -2.18074000 & 4.05080100 & -3.78120100 \\
\hline $\mathrm{H}$ & -3.31478500 & 2.22073900 & -3.72533000 \\
\hline $\mathrm{H}$ & -1.85252600 & 3.63582500 & 0.05502700 \\
\hline $\mathrm{H}$ & -0.82554500 & 5.58447700 & -1.06952500 \\
\hline $\mathrm{H}$ & -2.28056600 & 4.16371000 & -4.85769000 \\
\hline $\mathrm{H}$ & -1.02612700 & 5.85718800 & -3.53255400 \\
\hline $\mathrm{C}$ & 5.42511500 & -1.80800300 & -0.46872000 \\
\hline $\mathrm{C}$ & 6.85153400 & -4.02470500 & -1.46175100 \\
\hline $\mathrm{C}$ & 5.77757200 & -1.88689500 & -1.82387400 \\
\hline $\mathrm{C}$ & 5.80496800 & -2.85757200 & 0.38051100 \\
\hline $\mathrm{C}$ & 6.51353200 & -3.95471100 & -0.10927900 \\
\hline $\mathrm{C}$ & 6.48026400 & -2.98615200 & -2.31756800 \\
\hline $\mathrm{H}$ & 5.49964100 & -1.07862200 & -2.49428000 \\
\hline $\mathrm{H}$ & 5.53878800 & -2.81225300 & 1.43289700 \\
\hline $\mathrm{H}$ & 6.80071600 & -4.75580500 & 0.56714500 \\
\hline $\mathrm{H}$ & 6.74164400 & -3.02813200 & -3.37187400 \\
\hline $\mathrm{H}$ & 7.40259600 & -4.87950200 & -1.84509100 \\
\hline $\mathrm{C}$ & 6.71186100 & 0.79907100 & -0.35759300 \\
\hline $\mathrm{C}$ & 9.35708200 & 1.12065500 & -1.26858000 \\
\hline $\mathrm{C}$ & 7.78499500 & 0.21816700 & 0.33344300 \\
\hline $\mathrm{C}$ & 6.98824500 & 1.54479000 & -1.51256100 \\
\hline $\mathrm{C}$ & 8.29775700 & 1.70261200 & -1.96638000 \\
\hline $\mathrm{C}$ & 9.09568900 & 0.37878800 & -0.11551100 \\
\hline $\mathrm{H}$ & 7.58869000 & -0.36655500 & 1.22770900 \\
\hline $\mathrm{H}$ & 6.16862900 & 2.00510000 & -2.05704900 \\
\hline $\mathrm{H}$ & 8.48959300 & 2.28308100 & -2.86519700 \\
\hline $\mathrm{H}$ & 9.91325300 & -0.07813700 & 0.43628600 \\
\hline $\mathrm{H}$ & 10.37806100 & 1.24463800 & -1.61999000 \\
\hline $\mathrm{C}$ & 5.29216900 & 3.06746100 & 0.77838100 \\
\hline $\mathrm{C}$ & 6.58579500 & 5.55828400 & 1.00429900 \\
\hline $\mathrm{C}$ & 6.28611700 & 3.27534700 & 1.74529000 \\
\hline $\mathrm{C}$ & 4.95829800 & 4.12947800 & -0.07350800 \\
\hline $\mathrm{C}$ & 5.60003600 & 5.36340100 & 0.03581800 \\
\hline $\mathrm{C}$ & 6.92621700 & 4.50932800 & 1.85963000 \\
\hline $\mathrm{H}$ & 6.55970600 & 2.46104200 & 2.41042900 \\
\hline $\mathrm{H}$ & 4.19095400 & 3.98522900 & -0.82900500 \\
\hline $\mathrm{H}$ & 5.32813800 & 6.17304800 & -0.63666300 \\
\hline $\mathrm{H}$ & 7.69293800 & 4.64998700 & 2.61724700 \\
\hline $\mathrm{H}$ & 7.08516500 & 6.51969700 & 1.09135400 \\
\hline $\mathrm{C}$ & 2.61027000 & 2.75794500 & 1.78143700 \\
\hline $\mathrm{C}$ & 1.36788200 & 4.96242900 & 3.02047600 \\
\hline $\mathrm{C}$ & 2.82943600 & 3.03866700 & 3.13797800 \\
\hline
\end{tabular}




$\begin{array}{lccc}\mathrm{C} & 1.75951300 & 3.60335600 & 1.05510800 \\ \mathrm{C} & 1.14444300 & 4.69586100 & 1.66888600 \\ \mathrm{C} & 2.21357800 & 4.12893900 & 3.75365200 \\ \mathrm{H} & 3.48895700 & 2.39421800 & 3.71307700 \\ \mathrm{H} & 1.57640800 & 3.40340800 & 0.00325400 \\ \mathrm{H} & 0.49266700 & 5.34275800 & 1.08705100 \\ \mathrm{H} & 2.39710200 & 4.32752500 & 4.80648700 \\ \mathrm{H} & 0.88888100 & 5.81330700 & 3.49769300 \\ \mathrm{H} & 1.17528400 & -5.69597800 & 0.11541800 \\ \mathrm{H} & -1.12135200 & -5.68558300 & -0.20508800 \\ \mathrm{C} & 1.17301300 & -4.80728400 & 2.68321100 \\ \mathrm{H} & 1.87257200 & -4.72725700 & 3.52248100 \\ \mathrm{H} & 1.01222300 & -5.87406800 & 2.49429800 \\ \mathrm{C} & -1.72467600 & -4.71057300 & 2.25470400 \\ \mathrm{H} & -1.58052600 & -5.78768800 & 2.11763700 \\ \mathrm{H} & -2.62706900 & -4.58260100 & 2.86226200 \\ \mathrm{~S} & -0.39103200 & -4.09748100 & 3.38873900 \\ \mathrm{C} & 1.77799300 & -4.65315100 & -2.31794300 \\ \mathrm{H} & 1.62759900 & -5.73288500 & -2.21178800 \\ \mathrm{H} & 2.68229900 & -4.51301500 & -2.91992200 \\ \mathrm{C} & -1.11608300 & -4.72526800 & -2.74861600 \\ \mathrm{H} & -1.81596600 & -4.61995800 & -3.58480100 \\ \mathrm{H} & -0.95754300 & -5.79734600 & -2.59016800 \\ \mathrm{~S} & 0.44935500 & -3.99923700 & -3.43345900 \\ \mathrm{H} & 1.22893900 & -0.57020700 & 2.43502500 \\ \mathrm{H} & -0.93993500 & -0.73631100 & -1.83498700 \\ \mathrm{C} & -0.09571000 & 0.74140100 & -0.52688200 \\ \mathrm{H} & -0.32537200 & 1.74757800 & -0.15886100 \\ \mathrm{H} & 0.84593800 & 0.82497600 & -1.08672400 \\ \mathrm{C} & 0.10274100 & -0.21606900 & 0.64984900 \\ \mathrm{H} & 0.27827400 & -1.22409900 & 0.26546400 \\ \mathrm{H} & -0.82350200 & -0.27344900 & 1.23500500\end{array}$

Compound 13d: $\quad E(R B 3 L Y P)=-3921.57857190$ a.u.

$\begin{array}{lrrr}\mathrm{C} & -1.95175700 & -3.66141700 & -1.15978300 \\ \mathrm{C} & -2.76413700 & -2.34153200 & 1.17541800 \\ \mathrm{C} & -1.71179300 & -4.21951300 & 0.09738100 \\ \mathrm{C} & -2.66938700 & -2.46443100 & -1.22956700 \\ \mathrm{C} & -3.10142500 & -1.80086300 & -0.07369800 \\ \mathrm{C} & -2.07073300 & -3.55481400 & 1.27360100 \\ \mathrm{H} & -2.89969800 & -2.03486200 & -2.20195900 \\ \mathrm{H} & -3.07091800 & -1.82473700 & 2.08086900 \\ \mathrm{C} & 1.45578900 & -4.27206100 & 0.18243700 \\ \mathrm{C} & 2.97367800 & -1.92850500 & 0.42205800\end{array}$




\begin{tabular}{|c|c|c|c|}
\hline $\mathrm{C}$ & 1.81667400 & -3.57176400 & -0.97169700 \\
\hline $\mathrm{C}$ & 1.76546600 & -3.78693900 & 1.45384500 \\
\hline $\mathrm{C}$ & 2.57832700 & -2.65158200 & 1.55655600 \\
\hline $\mathrm{C}$ & 2.53711300 & -2.38200200 & -0.83394300 \\
\hline $\mathrm{H}$ & 2.90925100 & -2.33095000 & 2.54063600 \\
\hline $\mathrm{H}$ & 2.80838700 & -1.82138500 & -1.72292400 \\
\hline $\mathrm{C}$ & 3.88968700 & -0.73785600 & 0.50739700 \\
\hline $\mathrm{C}$ & 5.64064100 & 1.48250000 & 0.50410200 \\
\hline $\mathrm{C}$ & 5.11790400 & -0.79302400 & -0.20129300 \\
\hline $\mathrm{C}$ & 3.55423300 & 0.42013700 & 1.25330500 \\
\hline $\mathrm{C}$ & 4.44094600 & 1.52274600 & 1.24271600 \\
\hline $\mathrm{C}$ & 5.98794100 & 0.31730900 & -0.21069900 \\
\hline $\mathrm{C}$ & -3.93379400 & -0.55734000 & -0.21627300 \\
\hline $\mathrm{C}$ & -5.55105800 & 1.71604900 & -0.62214900 \\
\hline $\mathrm{C}$ & -3.35310100 & 0.63491500 & -0.70561700 \\
\hline $\mathrm{C}$ & -5.30942300 & -0.60515900 & 0.09782500 \\
\hline $\mathrm{C}$ & -6.11978200 & 0.53437100 & -0.09997900 \\
\hline $\mathrm{C}$ & -4.17061400 & 1.76604400 & -0.91215800 \\
\hline $\mathrm{C}$ & -1.85177000 & 0.71408100 & -0.93028400 \\
\hline $\mathrm{H}$ & -1.62448000 & 1.43460500 & -1.72150800 \\
\hline $\mathrm{C}$ & 2.30413200 & 0.49553900 & 2.12421800 \\
\hline $\mathrm{H}$ & 2.59637700 & 0.93241000 & 3.08561700 \\
\hline $\mathrm{C}$ & 5.53365600 & -2.04772400 & -0.91490800 \\
\hline $\mathrm{C}$ & 6.33820800 & -4.40387900 & -2.23402400 \\
\hline $\mathrm{C}$ & 5.65030800 & -2.08537600 & -2.31210800 \\
\hline $\mathrm{C}$ & 5.83361500 & -3.21095600 & -0.19075000 \\
\hline $\mathrm{C}$ & 6.23386800 & -4.37753400 & -0.84234700 \\
\hline $\mathrm{C}$ & 6.04378800 & -3.25256900 & -2.96690000 \\
\hline $\mathrm{H}$ & 5.43144500 & -1.19123300 & -2.88868900 \\
\hline $\mathrm{H}$ & 5.74647800 & -3.19983300 & 0.89220400 \\
\hline $\mathrm{H}$ & 6.46415800 & -5.26684500 & -0.26105400 \\
\hline $\mathrm{H}$ & 6.12564200 & -3.25981400 & -4.05082400 \\
\hline $\mathrm{H}$ & 6.64903300 & -5.31247800 & -2.74304400 \\
\hline $\mathrm{C}$ & 7.29704200 & 0.25614200 & -0.94226100 \\
\hline $\mathrm{C}$ & 9.75886700 & 0.15133200 & -2.30567400 \\
\hline $\mathrm{C}$ & 7.50968800 & 1.01735500 & -2.10043500 \\
\hline $\mathrm{C}$ & 8.34093700 & -0.55718700 & -0.47824100 \\
\hline $\mathrm{C}$ & 9.56124200 & -0.60845300 & -1.15162000 \\
\hline $\mathrm{C}$ & 8.72770000 & 0.96431500 & -2.77835900 \\
\hline $\mathrm{H}$ & 6.71298000 & 1.65606700 & -2.47100200 \\
\hline $\mathrm{H}$ & 8.19115100 & -1.15748400 & 0.41465300 \\
\hline $\mathrm{H}$ & 10.35781200 & -1.24456300 & -0.77416800 \\
\hline $\mathrm{H}$ & 8.87057500 & 1.56079900 & -3.67579100 \\
\hline $\mathrm{H}$ & 10.70890600 & 0.11045400 & -2.83198700 \\
\hline $\mathrm{C}$ & 6.58220500 & 2.65175300 & 0.53172800 \\
\hline $\mathrm{C}$ & 8.36107400 & 4.83309900 & 0.58282400 \\
\hline
\end{tabular}




$\begin{array}{lccc}\mathrm{C} & 6.34421000 & 3.79026900 & -0.25055800 \\ \mathrm{C} & 7.72708500 & 2.62489300 & 1.33998900 \\ \mathrm{C} & 8.60859700 & 3.70563200 & 1.36727100 \\ \mathrm{C} & 7.22574000 & 4.87129900 & -0.22775300 \\ \mathrm{H} & 5.46315600 & 3.82565100 & -0.88563900 \\ \mathrm{H} & 7.92790700 & 1.74744600 & 1.94843000 \\ \mathrm{H} & 9.49044000 & 3.66523900 & 2.00152700 \\ \mathrm{H} & 7.02447500 & 5.74376000 & -0.84421000 \\ \mathrm{H} & 9.04819200 & 5.67494700 & 0.60232200 \\ \mathrm{C} & 4.15744200 & 2.73496600 & 2.08707000 \\ \mathrm{C} & 3.68101200 & 5.00171900 & 3.69190300 \\ \mathrm{C} & 4.59587300 & 2.77913900 & 3.41893200 \\ \mathrm{C} & 3.48006800 & 3.84968500 & 1.57364000 \\ \mathrm{C} & 3.24397900 & 4.97360100 & 2.36703600 \\ \mathrm{C} & 4.35851400 & 3.89975900 & 4.21585800 \\ \mathrm{H} & 5.13059900 & 1.92653200 & 3.82984000 \\ \mathrm{H} & 3.13897700 & 3.83625000 & 0.54201600 \\ \mathrm{H} & 2.71910500 & 5.82801600 & 1.94746700 \\ \mathrm{H} & 4.70727800 & 3.91242900 & 5.24530100 \\ \mathrm{H} & 3.49773000 & 5.87640100 & 4.31029900 \\ \mathrm{C} & -5.91109300 & -1.86329600 & 0.65300900 \\ \mathrm{C} & -7.03238500 & -4.21996500 & 1.71126900 \\ \mathrm{C} & -6.31850900 & -1.92585900 & 1.99350900 \\ \mathrm{C} & -6.07709400 & -3.00215800 & -0.14816200 \\ \mathrm{C} & -7.31177400 & 2.88831900 & -1.98888000 \\ \mathrm{C} & -6.36501300 & 4.05622800 & -0.10736100 \\ \mathrm{C} & -6.63454400 & -4.16926200 & 0.37437000 \\ \mathrm{H} & -6.87141300 & -3.09353600 & 2.51962500 \\ \mathrm{H} & -6.20287300 & -1.04991100 & 2.62563400 \\ \mathrm{H} & -5.76429700 & -2.97175500 & -1.18806800 \\ \mathrm{H} & -6.75775600 & -5.04000400 & -0.26472300 \\ \mathrm{H} & -7.17967900 & -3.12119000 & 3.56168300 \\ \mathrm{H} & -7.46609800 & -5.12919700 & 2.11936200 \\ \mathrm{C} & -7.58338500 & 0.48548400 & 0.22539900 \\ \mathrm{C} & -10.33032800 & 0.39608800 & 0.84082900 \\ \mathrm{C} & -8.46514000 & -0.29594500 & -0.53527900 \\ \mathrm{C} & -8.10356400 & 1.22218700 & 1.29944100 \\ \mathrm{H} & -9.46326500 & 1.17637900 & 1.60709100 \\ \mathrm{H} & -9.82613500 & -0.33901700 & -0.23314900 \\ \mathrm{H} & -8.07896500 & -0.87537900 & -1.36912000 \\ \mathrm{H} & -7.43523100 & 1.83591300 & 1.89686900 \\ \mathrm{H} & -10.49252200 & -0.94930500 & -0.83740500 \\ \mathrm{H} & -6.42084500 & 2.90565100 & -0.90618200 \\ \mathrm{H} & -8.05625500 & 5.12899800 & -1.46403900 \\ \mathrm{H} & & & \\ \mathrm{H} & & & \end{array}$




\begin{tabular}{|c|c|c|c|}
\hline $\mathrm{C}$ & -7.17623400 & 5.15764600 & -0.38128900 \\
\hline $\mathrm{C}$ & -8.12052800 & 3.98992400 & -2.26787400 \\
\hline $\mathrm{H}$ & -7.37056700 & 2.00199600 & -2.61442800 \\
\hline $\mathrm{H}$ & -5.68162900 & 4.08626000 & 0.73651200 \\
\hline $\mathrm{H}$ & -7.11921400 & 6.03907700 & 0.25234900 \\
\hline $\mathrm{H}$ & -8.80275600 & 3.95665300 & -3.11349900 \\
\hline $\mathrm{H}$ & -8.68736100 & 5.98727100 & -1.67928600 \\
\hline $\mathrm{C}$ & -3.58071200 & 3.01766700 & -1.49938100 \\
\hline $\mathrm{C}$ & -2.47402600 & 5.34716000 & -2.63323700 \\
\hline $\mathrm{C}$ & -3.62710700 & 3.23514100 & -2.88433100 \\
\hline $\mathrm{C}$ & -2.97292700 & 3.99062800 & -0.69288200 \\
\hline $\mathrm{C}$ & -2.42392600 & 5.14510000 & -1.25348600 \\
\hline $\mathrm{C}$ & -3.07862900 & 4.38793700 & -3.44732900 \\
\hline $\mathrm{H}$ & -4.09831000 & 2.49225500 & -3.52229300 \\
\hline $\mathrm{H}$ & -2.93592600 & 3.84343800 & 0.38307500 \\
\hline $\mathrm{H}$ & -1.95910300 & 5.88759400 & -0.60977700 \\
\hline $\mathrm{H}$ & -3.12516200 & 4.53651400 & -4.52310900 \\
\hline $\mathrm{H}$ & -2.04733200 & 6.24589000 & -3.07071800 \\
\hline $\mathrm{H}$ & -1.22502700 & -5.18806700 & 0.16524900 \\
\hline $\mathrm{H}$ & 0.91896300 & -5.21125000 & 0.08741400 \\
\hline $\mathrm{C}$ & -1.43351200 & -4.31735100 & -2.41537500 \\
\hline $\mathrm{H}$ & -2.19705300 & -4.31613000 & -3.20052100 \\
\hline $\mathrm{H}$ & -1.15253700 & -5.36082900 & -2.23735600 \\
\hline $\mathrm{C}$ & 1.48364900 & -4.13177100 & -2.33472800 \\
\hline $\mathrm{H}$ & 1.37256600 & -5.22044000 & -2.28439700 \\
\hline $\mathrm{H}$ & 2.29943300 & -3.92520700 & -3.03508700 \\
\hline S & 0.00241900 & -3.46080100 & -3.22437100 \\
\hline $\mathrm{C}$ & -1.73357400 & -4.16592200 & 2.61358700 \\
\hline $\mathrm{H}$ & -1.65275900 & -5.25531000 & 2.53356700 \\
\hline $\mathrm{H}$ & -2.52452200 & -3.95470900 & 3.34062300 \\
\hline $\mathrm{C}$ & 1.20823100 & -4.44949500 & 2.68877500 \\
\hline $\mathrm{H}$ & 1.95487400 & -4.48617900 & 3.48921400 \\
\hline $\mathrm{H}$ & 0.89332300 & -5.47916200 & 2.48893900 \\
\hline S & -0.21388000 & -3.55371000 & 3.49206400 \\
\hline $\mathrm{H}$ & -1.47250800 & -0.25280600 & -1.26892800 \\
\hline $\mathrm{H}$ & 1.94087900 & -0.50666500 & 2.34659900 \\
\hline $\mathrm{C}$ & 1.08882100 & 1.29650700 & 1.60927800 \\
\hline $\mathrm{H}$ & 1.35662500 & 2.34565400 & 1.44608700 \\
\hline $\mathrm{H}$ & 0.36730000 & 1.29867900 & 2.43885800 \\
\hline $\mathrm{C}$ & 0.38763700 & 0.73461900 & 0.36130600 \\
\hline $\mathrm{H}$ & 0.46949900 & -0.35925100 & 0.34025900 \\
\hline $\mathrm{H}$ & 0.87979400 & 1.09787700 & -0.55161600 \\
\hline $\mathrm{C}$ & -1.09898200 & 1.11193000 & 0.35813700 \\
\hline $\mathrm{H}$ & -1.21249100 & 2.19114900 & 0.52453200 \\
\hline $\mathrm{H}$ & -1.57314100 & 0.62002300 & 1.21790000 \\
\hline
\end{tabular}


Compound 13e: $\quad \mathrm{E}(\mathrm{RB} 3 \mathrm{LYP})=-3960.89700111$ a.u.

$\begin{array}{lrrr}\mathrm{C} & -2.04190200 & -3.26852900 & -1.22765600 \\ \mathrm{C} & -3.04247000 & -2.15574400 & 1.13907700 \\ \mathrm{C} & -1.70379200 & -3.80568700 & 0.01696200 \\ \mathrm{C} & -2.93839800 & -2.19526500 & -1.26864400 \\ \mathrm{C} & -3.47181500 & -1.64698400 & -0.09322300 \\ \mathrm{C} & -2.14403700 & -3.22749000 & 1.20920700 \\ \mathrm{H} & -3.23627100 & -1.78132500 & -2.22968300 \\ \mathrm{H} & -3.42508100 & -1.71631700 & 2.05674900 \\ \mathrm{C} & 1.52854800 & -3.64918800 & 0.21981000 \\ \mathrm{C} & 3.33742200 & -1.52254700 & 0.21942900 \\ \mathrm{C} & 1.97366600 & -3.13887000 & -1.00073000 \\ \mathrm{C} & 1.88258200 & -3.06171700 & 1.43667100 \\ \mathrm{C} & 2.80128000 & -2.00525300 & 1.42320700 \\ \mathrm{C} & 2.88339100 & -2.07510000 & -0.98575200 \\ \mathrm{H} & 3.13001400 & -1.56836500 & 2.36380700 \\ \mathrm{H} & 3.27556900 & -1.69206600 & -1.92426100 \\ \mathrm{C} & 4.42484900 & -0.48280900 & 0.21805900 \\ \mathrm{C} & 6.50806100 & 1.41930000 & 0.21403200 \\ \mathrm{C} & 5.74332300 & -0.87861000 & -0.10148800 \\ \mathrm{C} & 4.13899300 & 0.86675600 & 0.51960100 \\ \mathrm{C} & 5.18665700 & 1.81278300 & 0.51456000 \\ \mathrm{C} & 6.78708700 & 0.07276500 & -0.10458700 \\ \mathrm{C} & -4.48755100 & -0.53967400 & -0.15986600 \\ \mathrm{C} & -6.41146600 & 1.51375800 & -0.30302300 \\ \mathrm{C} & -4.07520900 & 0.78570500 & -0.42208700 \\ \mathrm{C} & -5.85037700 & -0.83671000 & 0.05176800 \\ \mathrm{C} & -6.81618600 & 0.19152500 & -0.01940400 \\ \mathrm{C} & -5.04368700 & 1.80840600 & -0.49211600 \\ \mathrm{C} & -2.59750700 & 1.09607600 & -0.58628200 \\ \mathrm{C} & 2.71491600 & 1.30070400 & 0.81894800 \\ \mathrm{H} & 2.72559600 & 2.20450200 & 1.43584000 \\ \mathrm{C} & 6.03944100 & -2.30844800 & -0.45009900 \\ \mathrm{C} & 6.60280500 & -4.98716200 & -1.11411400 \\ \mathrm{C} & 6.38586400 & -2.66625500 & -1.76119300 \\ \mathrm{C} & 5.98224100 & -3.31705500 & 0.52247600 \\ \mathrm{C} & 6.26320700 & -4.64378400 & 0.19536800 \\ \mathrm{C} & 6.66222300 & -3.99281500 & -2.09193100 \\ \mathrm{H} & 6.44290100 & -1.89579800 & -2.52491900 \\ \mathrm{H} & 5.71616200 & -3.05736500 & 1.54318700 \\ \mathrm{H} & 6.21759900 & -5.40949200 & 0.96572500 \\ \mathrm{H} & 6.92834300 & -4.24839100 & -3.11445100 \\ \mathrm{H} & 6.82197400 & -6.02051000 & -1.37003700 \\ \mathrm{C} & 8.18927200 & -0.34505600 & -0.43540900 \\ \mathrm{C} & 10.82319400 & -1.12277900 & -1.05926900 \\ & & & \end{array}$




\begin{tabular}{|c|c|c|c|}
\hline $\mathrm{C}$ & 8.80866400 & 0.09577100 & -1.61398000 \\
\hline $\mathrm{C}$ & 8.91396300 & -1.18250600 & 0.42499300 \\
\hline $\mathrm{C}$ & 10.21938000 & -1.56619500 & 0.11841000 \\
\hline $\mathrm{C}$ & 10.11207800 & -0.29085500 & -1.92540400 \\
\hline $\mathrm{H}$ & 8.26281200 & 0.74793600 & -2.28989700 \\
\hline $\mathrm{H}$ & 8.44814000 & -1.53625300 & 1.34036400 \\
\hline $\mathrm{H}$ & 10.76435500 & -2.21370700 & 0.80059600 \\
\hline $\mathrm{H}$ & 10.57242700 & 0.06023300 & -2.84543300 \\
\hline $\mathrm{H}$ & 11.83972300 & -1.42285500 & -1.29988400 \\
\hline $\mathrm{C}$ & 7.62165000 & 2.42337600 & 0.27602200 \\
\hline $\mathrm{C}$ & 9.71556600 & 4.30095300 & 0.41686900 \\
\hline $\mathrm{C}$ & 7.75157000 & 3.42787400 & -0.69330200 \\
\hline $\mathrm{C}$ & 8.56028800 & 2.37595500 & 1.31683400 \\
\hline $\mathrm{C}$ & 9.59644800 & 3.30677800 & 1.38914400 \\
\hline $\mathrm{C}$ & 8.78972900 & 4.35731600 & -0.62589500 \\
\hline $\mathrm{H}$ & 7.03391300 & 3.47873000 & -1.50717800 \\
\hline $\mathrm{H}$ & 8.47674200 & 1.60068000 & 2.07331400 \\
\hline $\mathrm{H}$ & 10.31225400 & 3.25335500 & 2.20545500 \\
\hline $\mathrm{H}$ & 8.87378900 & 5.12657100 & -1.38932400 \\
\hline $\mathrm{H}$ & 10.52366700 & 5.02572100 & 0.47097700 \\
\hline $\mathrm{C}$ & 4.90651400 & 3.24207600 & 0.88506000 \\
\hline $\mathrm{C}$ & 4.37577700 & 5.91143100 & 1.61128700 \\
\hline $\mathrm{C}$ & 5.12123100 & 3.68585300 & 2.19828400 \\
\hline $\mathrm{C}$ & 4.42365600 & 4.16088500 & -0.05795800 \\
\hline $\mathrm{C}$ & 4.16019600 & 5.48393800 & 0.30060100 \\
\hline $\mathrm{C}$ & 4.85768100 & 5.00747200 & 2.55938200 \\
\hline $\mathrm{H}$ & 5.49844400 & 2.98671600 & 2.93972700 \\
\hline $\mathrm{H}$ & 4.25953600 & 3.83668600 & -1.08216400 \\
\hline $\mathrm{H}$ & 3.78775000 & 6.18033900 & -0.44633200 \\
\hline $\mathrm{H}$ & 5.03096400 & 5.33102600 & 3.58254200 \\
\hline $\mathrm{H}$ & 4.17105400 & 6.94128900 & 1.89167700 \\
\hline $\mathrm{C}$ & -6.27043800 & -2.24013600 & 0.37800700 \\
\hline $\mathrm{C}$ & -7.04867500 & -4.87186600 & 1.01054900 \\
\hline $\mathrm{C}$ & -6.70021600 & -2.57101700 & 1.67133100 \\
\hline $\mathrm{C}$ & -6.23854700 & -3.25139400 & -0.59268700 \\
\hline $\mathrm{C}$ & -6.62593500 & -4.55493000 & -0.28119400 \\
\hline $\mathrm{C}$ & -7.08381900 & -3.87452000 & 1.98643500 \\
\hline $\mathrm{H}$ & -6.73720500 & -1.79735300 & 2.43313800 \\
\hline $\mathrm{H}$ & -5.90818300 & -3.01243600 & -1.59961800 \\
\hline $\mathrm{H}$ & -6.59780700 & -5.32324400 & -1.04978600 \\
\hline $\mathrm{H}$ & -7.41345800 & -4.10967500 & 2.99524900 \\
\hline $\mathrm{H}$ & -7.35041600 & -5.88719000 & 1.25422700 \\
\hline $\mathrm{C}$ & -8.26657100 & -0.12084900 & 0.20147100 \\
\hline $\mathrm{C}$ & -10.98939000 & -0.70517100 & 0.62096700 \\
\hline $\mathrm{C}$ & -8.98627900 & -0.89288100 & -0.72205900 \\
\hline $\mathrm{C}$ & -8.93629000 & 0.35318400 & 1.33893200 \\
\hline
\end{tabular}




\begin{tabular}{|c|c|c|c|}
\hline $\mathrm{C}$ & -10.28413100 & 0.06232400 & 1.54911800 \\
\hline $\mathrm{C}$ & -10.33550100 & -1.18074000 & -0.51666400 \\
\hline $\mathrm{H}$ & -8.48240900 & -1.27052400 & -1.60726600 \\
\hline $\mathrm{H}$ & -8.39469900 & 0.95609200 & 2.06234400 \\
\hline $\mathrm{H}$ & -10.78358300 & 0.43777700 & 2.43861900 \\
\hline $\mathrm{H}$ & -10.87552400 & -1.77864800 & -1.24641200 \\
\hline $\mathrm{H}$ & -12.04024000 & -0.93064300 & 0.78273600 \\
\hline $\mathrm{C}$ & -7.43859700 & 2.59945000 & -0.43816100 \\
\hline $\mathrm{C}$ & -9.37267400 & 4.62746600 & -0.71293600 \\
\hline $\mathrm{C}$ & -8.29621100 & 2.62878900 & -1.54731700 \\
\hline $\mathrm{C}$ & -7.56679000 & 3.60499000 & 0.53036600 \\
\hline $\mathrm{C}$ & -8.52627700 & 4.60876000 & 0.39653600 \\
\hline $\mathrm{C}$ & -9.25352400 & 3.63362700 & -1.68555600 \\
\hline $\mathrm{H}$ & -8.21279700 & 1.85413100 & -2.30450200 \\
\hline $\mathrm{H}$ & -6.91018300 & 3.59802100 & 1.39573900 \\
\hline $\mathrm{H}$ & -8.61101800 & 5.37749400 & 1.16043600 \\
\hline $\mathrm{H}$ & -9.90754400 & 3.63851800 & -2.55379000 \\
\hline $\mathrm{H}$ & -10.11925100 & 5.41024900 & -0.81850200 \\
\hline $\mathrm{C}$ & -4.62424600 & 3.21436500 & -0.81484500 \\
\hline $\mathrm{C}$ & -3.83368700 & 5.84368900 & -1.44297400 \\
\hline $\mathrm{C}$ & -4.72112200 & 3.69945000 & -2.12724100 \\
\hline $\mathrm{C}$ & -4.12626300 & 4.07122000 & 0.17753700 \\
\hline $\mathrm{C}$ & -3.73471300 & 5.37462800 & -0.13245500 \\
\hline $\mathrm{C}$ & -4.32849000 & 5.00120900 & -2.43992400 \\
\hline $\mathrm{H}$ & -5.10842900 & 3.04830800 & -2.90616700 \\
\hline $\mathrm{H}$ & -4.05135700 & 3.71337700 & 1.20090700 \\
\hline $\mathrm{H}$ & -3.35320100 & 6.02338600 & 0.65189700 \\
\hline $\mathrm{H}$ & -4.41121000 & 5.35744300 & -3.46354500 \\
\hline $\mathrm{H}$ & -3.52880800 & 6.85828600 & -1.68540300 \\
\hline $\mathrm{H}$ & -1.07454900 & -4.68870700 & 0.05732800 \\
\hline $\mathrm{H}$ & 0.89059500 & -4.52511600 & 0.22454300 \\
\hline $\mathrm{C}$ & -1.43216500 & -3.82308700 & -2.49200700 \\
\hline $\mathrm{H}$ & -2.14035900 & -3.75822500 & -3.32423700 \\
\hline $\mathrm{H}$ & -1.16245600 & -4.87830000 & -2.37666900 \\
\hline $\mathrm{C}$ & 1.50836500 & -3.74590400 & -2.30204500 \\
\hline $\mathrm{H}$ & 1.25818100 & -4.80482200 & -2.17816900 \\
\hline $\mathrm{H}$ & 2.30166500 & -3.68777200 & -3.05364400 \\
\hline S & 0.06346300 & -2.92584600 & -3.14266900 \\
\hline $\mathrm{C}$ & -1.64714700 & -3.73830500 & 2.53994700 \\
\hline $\mathrm{H}$ & -1.32151100 & -4.78168600 & 2.47175300 \\
\hline $\mathrm{H}$ & -2.44257500 & -3.69519500 & 3.29056200 \\
\hline $\mathrm{C}$ & 1.29678300 & -3.57850300 & 2.72908000 \\
\hline $\mathrm{H}$ & 1.99212000 & -3.41479700 & 3.55852900 \\
\hline $\mathrm{H}$ & 1.10605100 & -4.65579600 & 2.67523100 \\
\hline S & -0.26715100 & -2.76591400 & 3.32862400 \\
\hline $\mathrm{H}$ & -2.11409300 & 0.27876500 & -1.12991900 \\
\hline
\end{tabular}




$\begin{array}{lrrr}\mathrm{H} & 2.21614400 & 0.52672000 & 1.40638800 \\ \mathrm{C} & 1.88080500 & 1.57224100 & -0.45109600 \\ \mathrm{H} & 1.85426700 & 0.66106100 & -1.06291200 \\ \mathrm{H} & 2.38448900 & 2.33758300 & -1.05457800 \\ \mathrm{C} & 0.43919900 & 2.02074900 & -0.16040400 \\ \mathrm{H} & -0.06646100 & 2.18042600 & -1.12214300 \\ \mathrm{C} & -1.86419800 & 1.27563400 & 0.76630000 \\ \mathrm{H} & -2.28886300 & 0.57904200 & 1.49801100 \\ \mathrm{C} & -0.35457900 & 1.01380400 & 0.68457700 \\ \mathrm{H} & 0.04586300 & 0.99812100 & 1.70595300 \\ \mathrm{H} & -0.19919300 & 0.00194300 & 0.28374800 \\ \mathrm{H} & -2.05660400 & 2.28515300 & 1.15381300 \\ \mathrm{H} & -2.47309200 & 1.99367300 & -1.19697500 \\ \mathrm{H} & 0.44820400 & 2.99752200 & 0.34598000\end{array}$

